\title{
ISOTOPE SEPARATION BY LASER DEFLECTION OF AN ATOMIC BEAM
}

Anthony F. Bernhardt

(Ph. D. Thesis)

February 1975

\section{MASTER}

Prepaned for U.S. Energy Research \& Development Administration under contract No. W-7405-Eng-48

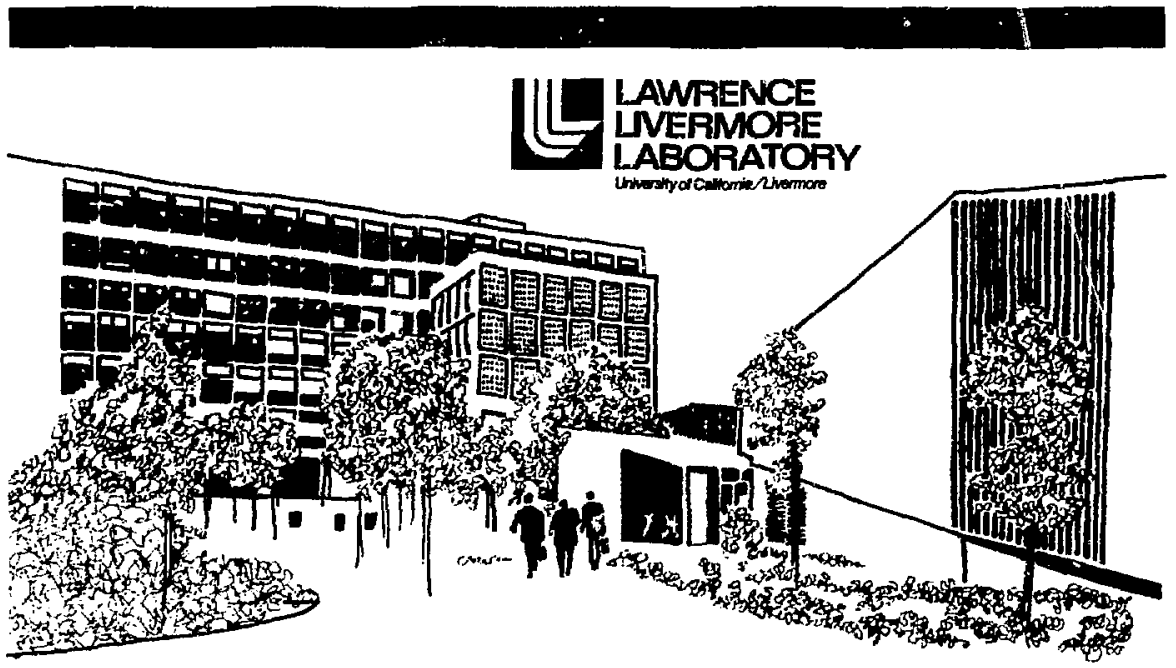


NOTICE

"This report was prepared as an account of work sponsored by the United States Government. Neither the United States nor the United States Energy Research \& Development Administration, uor amy of their emplnyecs, not any of their contractors. subcontractors, or their empluyes, makes any subcontractors, or their employees, makes any liability or responsibility for the sccuracy. completeness or usefulness of any information. appartus, product or process disclosed or represents that its use would not infringe privately-owned rights."

Printed in the United States of America Available from

National Technical Information Service

U.S. Department of Commerce 5285 Port Royal Road Springfield, Virginia 22151

Price: Printed Copy $\$$ *; Microfiche $\$ 2.25$

$$
\begin{gathered}
* \text { Pages } \\
\hline 1-50 \\
51-150 \\
151-325 \\
326-500 \\
501-1000
\end{gathered}
$$

NTIS

Selling Price

$\$ 4.00$

$\$ 5.45$

$\$ 7.60$

$\$ 10.60$

$\$ 13.60$ 


\title{
노 \\ LAWRENCE LVERMORE LABORATORY \\ unversity of Caffornia/Livemere,Caltomia/94550
}

\section{UCRI-51796 \\ ISOTOPE SEPARATION BY LASER DEFLECTION OF AN : 'OMIC BEAM}

\author{
Anthony F. Rernhardt \\ (Ph. D. Thesis)
}

MS. Date: February 1075
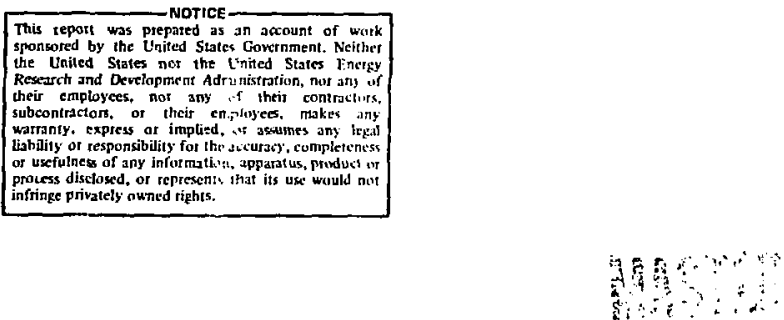
Anthony Frederick Bernhardt

February 1975

Applied Science

Isotope Separation by Laser Deflection of an Atomic Beam

\section{Abstract}

Separation of isotopes of barium has been accomplished by laser deflection of a single isotopic component of an atomic beam. With a tunable narrow linewidth dye laser, small differences in absorption frequency of different barium isotopes on the $6 s^{2}{ }^{1} s_{0}-6 s 6 p{ }^{1_{P_{1}}} 5536 \AA$ resonance were exploited to deflect atoms of a single isotopic component of an atomic beam through an angle Targe enough to physically separate them from the a tomic beam.

It is shown that the principal limitation on separation efficiency, the fraction of the desired isotopic component which can be separated, is determined by the branching ratio from the excited state into metastable states. In barium, repeated absorptions and emissions on the $5536 \AA$ transition eventually result in decay from the $6 s 6 p{ }^{1} P_{1}$ state to the metastable $6 s 5 d{ }^{1} D_{2}$ state. This was observed to occur for all but $3 \%$ of the ${ }^{138_{B a}}$ atoms. As a result, the efficiency of separation was about 0.7 for the $8 \mathrm{mrad}$ atomic beam divergence employed. (Throughput was nearly $l \mathrm{mg} /$ day. No attempt was made to maximize this value.)

An expression for the separation efficiency as a function of the branching ratio of the $6 s 6 p T_{P}$ level into the metastable ${ }_{D}$ level was derived. Using the experimental yalue for the separation efficiency, the branching ratio into the ${ }^{1}$ state was found to be $\delta \frac{1}{700}$, corresponding to an oscillator strength $f \lesssim 0.0034$. This is a drastic correction to the previously accepted experimental value for the branching ratio of $\frac{1}{24}$, 
al though it agrees relatively well with a more recent theoretical estimate of $\frac{1}{600}$.

The isotopic purity of the separated atoms was measured to be in excess of 0.9 , limited only by instrumental uncertainty. Neither Van der Waals scattering nor excitation exchange limit isotopic purity at practical atomic beam densities.

The photjdeflection process was used in a high resolution, mass sensitive spectroscopy experiment. A quadrupole mass spectrometer was used to monitor isotopic composition of the separated atoms. The various isotopic peak heights were plotted as a function of laser frequency. This produced a separate absorption curve for each isotope and absorption lines of different isotopes which could not be resolved by ordinary optical means were resolved using photodeflection. Thus, for example, ${ }^{134} \mathrm{Ba},{ }^{135} \mathrm{Ba} \mathrm{F}=\frac{5}{2}$ and ${ }^{136} \mathrm{Ba}$ peaks were resolved on the $5536 \AA$ resonance for the first time without the use of enriched samples. The relative position of isotopic absorption peaks in the $5536 \AA$ line were determined with an accuracy of better than $8 \mathrm{MHz}$. The accuracy of the experiment was limited by the electronic stability of the mass analyzer used and by the 1 imited number of runs performed. Better statistics and a more modern analyzer car. reduce the error considerably.

To improve the efficiency of separation, a second dye laser was empioyed to excite atoms which had decayed to the 6s5d metastable state into the $6 \mathrm{p} 5 \mathrm{~d}^{1} \mathrm{P}_{\mathrm{I}}$ state from which they could decayy to the ground state and continue to be deflected on the $5535 \AA$ transition. With the addition of the second laser, separation efficiency of greater than $83 \%$ was achieved, limited by metastable state accumulation in the $5 \mathrm{~d}^{2} \mathrm{I}_{2}$ state which is accessible from the $6 p 5 d{ }^{1} P_{1}$ level. It was found that the decay rate from the $6 \mathrm{p} 5 \mathrm{~d}$ state into the $5 \mathrm{~d}^{2}$ metastable state was fully $2 / 3$ the decay rate to the ground state, corresponding to an oscillator strength of 0.58 . 
TABLE OF CONTENTS

Page

Abstract ................. $\mathbf{i}$

Table of Contents. . . . . . . . . . . . ii i

1. Photodeflection Isotope Separation ........ 1

11. Photodeflection Applied to Barium Isotopes . . . . . 27

III. Dye Lasers and Optical Spectrum Analyzers. . . . . . 36

IV. Experimental Apparatus . . . . . . . . . 49

V. Experimental Results

A. Absorption Spectrum of Natural Barium on the $6 s^{2}{ }^{1} s_{0}-6 s 6 p{ }_{P_{1}} 5535 \AA$ Transition. . . . . . . . 54

B. Metastable State Accumulation. . . . . . . 56

C. Isotope Separation Without Metastable State Depopulation ............ 60

D. High Resolution Spectroscopy in Barium Using Photodeflection. . . . . . . . . . 65

E. Separation Efficiency Without Metastable State Depopulation ............ 68

i. Branching Ratio into the $655 d^{~} D_{2}$ Metastable State-A Theoretical Treatment of Deflection Efficiency ............. . 72

G. Branching Ratio and 0scillator Strength into the

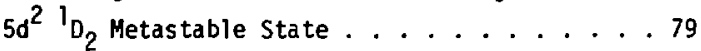

H. Separation Efficiency with Metastable State Depoputation ............ 87

VI. Extensions of the Photodeflection Method .....90 Acknowledgments. . . . . . . . . . . . . 95 Appendix A . . . . . . . . . . . . . 97 References ................... 101 Distribution ....................... . . . . 


\section{PHOTODEFLECTION ISOTOPE SEPARATION}

The photodeflection method of isotope separation relies on momentum transfer from a directed monochromatic light source to the one isotopic component of an atomic beam which is to be separated from other isotepic components of the baam. ${ }^{1,2}$ When an atom absorbs a photon of energy $h_{v}$ it acquires a momentum $h v / c$ in the direction of the photon propagation. If the atom decays spontaneously, it will give up momentum hu/c to the departing photon in the direction of its propagation. Since the direction of spontaneous radiation is random, the average net momentum transfer from $N$ emission events is $\frac{h v}{c \sqrt{N}}$. Thus momentum contributions from the emission process tand to zero whereas, contributions from the absorption process with a directed light source sum.

The isotopic selectivity of the photodeflection isotope separation process derives from the fact that different isotopes absorb light at slightly different frequencies. Thus a given electronic transition will occur in one isotope at a certain frequency and at slightly different frequency in another. The difference in absorption frequency is designated the isotope shift for a given pair of isotopes considered. The isotope shift is usually large compared to natural linewidth of the transitionthe frequency uncertainty resulting from the finite lifetine of the transition. ${ }^{3}$ If isotope shifts were much smaller than the natural linewidth, the isotopes could not. be distinguished optically except under special circumstances ${ }^{4}$ and would not be separable by photodeflection. The lifetime of an excited state $m$ is given by ${ }^{5}$

$$
\frac{1}{\tau}=\frac{8 \pi e^{2}}{m_{e} c} \sum_{n} \frac{g_{n}}{g_{m}} \frac{f_{n}}{\lambda_{n m}^{2}}
$$


where $m_{e}$ is the electron mass, $g_{m}$ and $g_{n}$ are the degeneracies of the upper and lower states, respectively (the number of states with energy $E_{m}$ and $E_{n}$, respectively), $f_{n}$ is the oscillator strength of the transition $m \cdot n$, and the summation runs over fli lower states $n$. For a classical oscillator $f=1$ and for a strong electronic transition in an atom $f_{n} \approx 1$. For such a transition $g_{n}=2 L_{n}+1$ and $g_{m}=2 L_{m}+1$ where $L_{n}=L_{m} \pm 1$ and $L_{m}, L_{n} \geq 0$ so that

$$
\frac{g_{n}}{g_{m}}=\left|\frac{2\left(L_{m} \pm 1\right)+1}{2 L_{m}+1}\right|=\left|1 \pm \frac{2}{2 L_{m}+1}\right|
$$

and therefore

$$
\frac{1}{3} \leq \frac{g_{n}}{g_{m}} \leq 3
$$

Thus if all the terms in the expression for $\frac{1}{T}$ are negligible save one

$$
\frac{8 \pi e^{2}}{3 m e^{c}} \frac{1}{\lambda^{2}} \therefore \frac{1}{\tau} \leqslant \frac{8 \pi e^{2}}{m e^{c}} \frac{3}{\lambda^{2}}
$$

For photons with $\lambda=5500 \AA$ this condition becomes

$$
2 \times 10^{7} \lesssim \frac{1}{\tau} \lesssim 2 \times 10^{8}
$$

and

$$
3.3 \times 10^{6} \approx \Delta \nu=\frac{1}{2 \pi \tau} \lesssim 3.3 \times 10^{7}
$$


gives the corresponding range of natural linewidths. For light e? ements the isotope shift is the result of the fact that the reduced masses of the isotopes are different. ${ }^{6}$ For a hydrogen-like atom

$$
E_{a}=-\mu_{a} \frac{e^{4}}{2 \pi^{2} n^{2}}
$$

where $\mu_{a}$ is the reduced mass of isotope a. Since $\mu_{a} \sim m_{e}\left(1-\frac{m_{e}}{m_{a}}\right)$ the energy level is displaced toward the continuum for finite nuclear mass, $M_{a}$. The displacement is larger for the ligh:ter isotope. The difference in transition energy for two isotopes is

$$
h v_{b}-h v_{a}=\left(\mu_{b}-\mu_{a}\right) \frac{e^{4}}{2 \hbar^{2}}\left(\frac{1}{n_{2}^{2}}-\frac{1}{n_{1}^{2}}\right) \simeq \frac{M_{b}-M_{a}}{M_{b} M_{a}} \frac{m_{e} e^{4}}{2 \hbar^{2}}
$$

From these considerations it is seen that the magnitude of the shift in a hydrogen-like atom is given by $\Delta v \sim \frac{m_{e}}{m_{p}} \frac{A_{2}-A_{1}}{A_{2} A_{1}} v$ so that $\Delta v \sim 5 \times 10^{-4} \frac{v}{A^{2}}$. This so called "normal mass effect" is chardcterized by the fact that the lighter isotope transition is less energetic than the heavy isctope transiticn $\left(\mu_{b}>\mu_{a} \Rightarrow v_{b}>v_{a}\right)$ and that the magnitude of the energy difference is inversely proportional to the square of the atomic weight, A.

For atoms which have more than one electron outside of a closed shell, the mass dependent isotope shift can become more complicated. Consider, for example, an atom with two electrons. The kinetic energy of the system is

$$
\frac{P_{n}^{2}}{2 M}+\frac{1}{2 m_{e}}\left(P_{1}^{2}+P_{2}^{2}\right)
$$


where $M$ and $\vec{P}_{n}$ are the mass and momentum of the nucleus. $\vec{P}_{1}$ and $\vec{P}_{2}$ are the momenta of the two electrons. In the center of mass system, the total momentum is zero so that

$$
\overrightarrow{\mathrm{P}}_{\mathrm{n}}=-\left(\overrightarrow{\mathrm{P}}_{1}+\overrightarrow{\mathrm{P}}_{2}\right) \text { and } \mathrm{P}_{\mathrm{n}}^{2}=\mathrm{P}_{1}^{2}+\mathrm{P}_{2}^{2}+2 \overrightarrow{\mathrm{P}}_{1} \cdot \overrightarrow{\mathrm{P}}_{2}
$$

The kinetic energy can then be expressed as

$$
\frac{1}{2 n}\left(p_{1}^{2}+p_{2}^{2}\right)+\frac{1}{M} \vec{p}_{1} \cdot \vec{p}_{2}
$$

The first term gives rise to the "normal mass effect" discussed above while the second term is new. It gives rise to what is referred to as the "specific mass effect". The property of the specific mass effeci which is most apparent is that it can have either sign. For $\vec{P}_{1}$ parallel to $\overrightarrow{\mathrm{P}}_{2}$ (on the average) it is positive. For opposed electron motions (on the average) the term has a negative sign. A quantum mechanical treatment of the problem ${ }^{7}$ shows that the positive sign applies to singlet terms and the negative sign to triplet terms. Another quantum mechanical result is that the specific mass effect is nonzero only for electron pairs with azimuthal quantum rumbers $\ell$ differing by one. The fractional shift in an energy level is again on the order of the electron-proton mass ratio and the isotope shift is also inversely proportional to the square of the atomic weight. The $\frac{1}{A^{2}}$ dependence makes mass effects negligible in heavy isotope shifts $(A \geq 140)$. Experimental values of isotope shifts in the resonance lines of alkali-like spectra are compared with natural linewidth in Table 1. A similar comparison is made for alkali earth and related elements in Table 2. The increasing shift and change of sign in the heavier elements is due to volume effects discussed below. 
TABLE 1

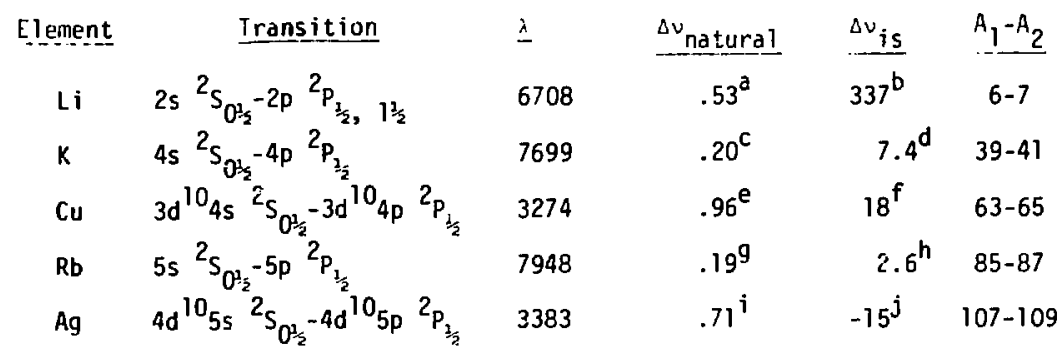

a. M. G. Veselov and A. V. Shtoff, Opt. \& Spectrosc. 26, 177 (1970).

b. R. H. Hughes, Phys. Rev. 59, 1837 (1955).

c. V. I. Ostroyskii and N. P. Penkin, Opt. Spectrosc. 12, 379 (1962).

d. E. C. Wang and J. Yellin, Phys. Rev. A4, 838 (1971).

e. M. Kock and J. Richter, Z. Astrophysik 69, 180(1968).

f. P. Brix and W. Humbach, Z. Phys. $128,5 \overline{06}$ (1950).

9. E. L. A7tman and S. A. Kanzantsev, Opt. Spectrosc. 28, 432 (1971).

h. H. M. Gibbs and G. C. Churchill, J. Opt. Soc. Am. 62, 1130 (1972).

i. P. T. Cunningham and J. K. Link, J. 0pt. Soc. Am. 57, 1000 (1967).

j. P. Brix, et al.. Z. Phys. 130, 88 (1951).

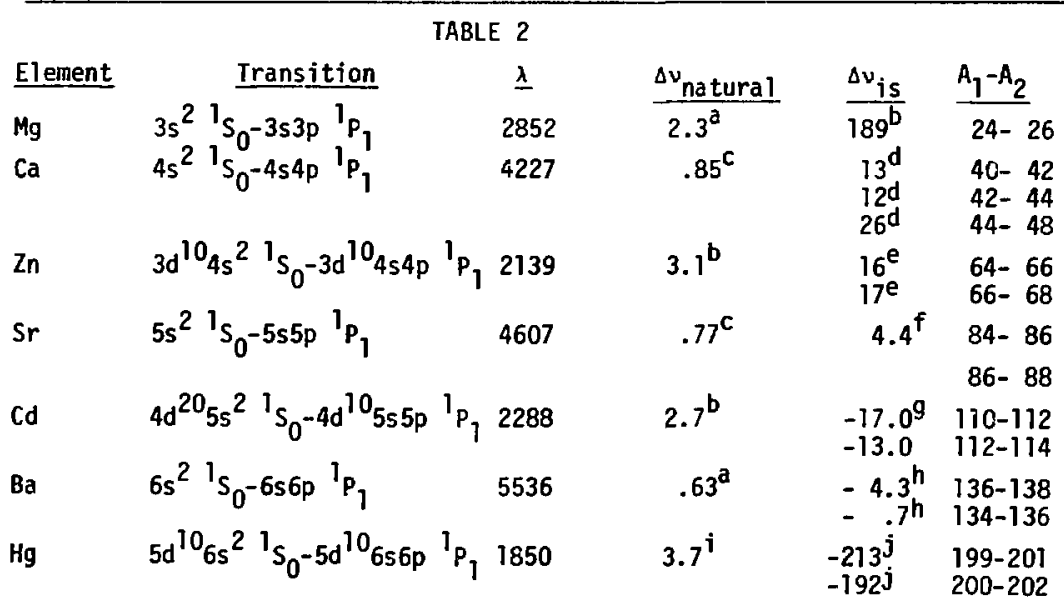

a. A. Lurio, Phys. Rev. 136, A376 (1964).

b. Landholt-Bornstein, Atom und Molekularphysik 1. Teil, Springer-Vriag

c. Y.I. Ostrovskii and N.P. Penkin, Opt. Spectrosc. 11, 307 (1961).

d. G.L. Epstein and S.P. Davis, Phys. Rev. A4, 464 (1971).

e. M.F. Crawford, et al, Can. J. Res. 28A, $1 \overline{3} 8$ (1950).

f. K. Hellig, Z. Phys. 161, 252 (1961).

g. F.M. Kelly and E. Tomchuk, Proc. Phys. Soc. 76, 1304 (1961).

h. This work.

i. A. Lurio, Phys. Rev. 140, Al505 (1966).

j. Landolt-Bornstein, Atom und Molekularphysik 5. Teil, Springer-Verlag 
For heavy atoms, the dominant effect in the isotope shift is the change in nuclear charge distribution on addition of a neutron to that nucleus. This effect is important orily when the optical transition invoives an s electron, since only an s electron has a non-negligible wavefunction at the nucleus. Inside the nucleus an s electron no longer sees a $\frac{1}{r}$ potential. If one assumes a uniform charge distribution inside a spherical nucleus, the potential is given by $\phi=\frac{z e}{r_{0}}\left(-\frac{3}{2}+\frac{1}{2} \frac{r^{2}}{r_{0}^{2}}\right)$, where $r_{0}$ is the nuclear radius. The potentials for two isotopes, differing by $\delta r_{0}$ in nuclear radius are represented in Figure 1 . The smailer nucleus has the iarger potential and the electron is more strongly bound by it since it is closer to the $\frac{1}{r}$ potential of a point charge. The difference in potential is given by

$$
\delta_{\phi}=-\frac{3}{2} \frac{Z \mathrm{e}}{r_{0}^{2}} \quad \hat{o} r_{0}\left(1-\frac{r^{2}}{r_{0}^{2}}\right)
$$

The difference in energy of an s electron in a pair of isotopes is

$$
\delta E=\int_{0}^{r_{0}} \rho_{e} \delta \phi d V
$$

where

$$
o_{e}=-e\left|\psi_{s}(0)\right|^{2} \sim-\frac{e}{\pi a_{0}^{3}} \frac{z}{n^{3}}
$$

(where $a_{0}$ is the Bohr radius). Then,

$$
\begin{aligned}
\delta E \approx \frac{3}{2} \frac{z^{2} e^{2}}{\pi a_{0}^{3} n^{3}} & \frac{\delta r_{0}}{r_{0}} \int_{0}^{r_{0}}\left(1-\frac{r^{2}}{r_{0}^{2}}\right) 4 \pi r^{2} d r \\
& \approx \frac{r_{0}^{2} \delta r_{0}}{a_{0}^{2} r_{0}} \frac{z^{2}}{n^{3}} R_{\infty} \backsim \frac{r_{0}^{2}}{a_{0}^{2}} \frac{z^{2}}{n^{3}} \frac{\delta A}{A} R_{\infty}
\end{aligned}
$$




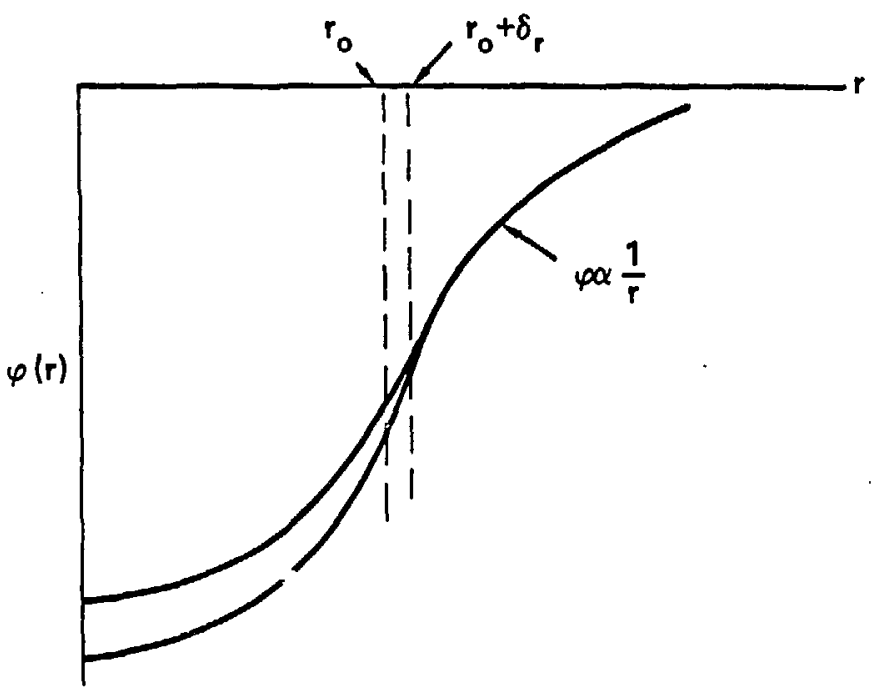

FIGURE 1: The potential of two isotopes in the approximation of a spherical, uniformly charged nucleus. The heavier nucleus has the larger radius and the shallower potential well inside the nucleus. The smaller nucleus deviates less from a $1 / \vec{r}$ potential and binds electrons more strongly than the heavier nucleus. 
where $R_{\infty}$ is the Rydberg constant. Thus the isotope shift due to the change in nuclear volume decreases inversely with atomic weight but the heavy isotope always lies higher in energy (closer to the continuum). Therefore, an electronic transition from a p orbital to a lower s orbital will be less energetic for the heavier isotope. A transition from an $s$ orbital to a lower p orbital will be less energetic for the lighter isotope.

The assumption of a spherical nucleus which merely increases in radius when a neutron is added with $r_{0} \propto A^{1 / 3}$ ignores the fact the nuclei also change shape when neutrons are added. In Figure 2 nuclear deformation, defined by the parameter $\beta^{2}=\left(\frac{a-b}{r_{0}}\right)^{2}$ where $a$ and $b$ are semimajor and semiminor axes of the nuclear ellipsoid of equivalent spherical radius $r_{0}$, is plotted against neutron number. It is apparent that only near the magic numbers $N=50,82$ and 126 does $\beta^{2}$ approach zero (except for Sn which has a magic number of protons). An isotope shift results from a change in nuclear shape as well as from a change in nuclear volume. The potential of an ellipsoidal uniformly charged nucleus, avaraged over direction, is equivalent to the potential of a charge distribution similar to Figure 3a. The charge density is zero outside $r=a$. Inside $r=a$ it gradually increases until at $r=b$ it becomes $o=\frac{Z}{V_{n}}$ where $Z$ is the total nuclear charge and $V_{n}$ is the volume of the nuclear ellipsoid. The potential which results from such a distribution is shown in Figure 3b. For comparison a spherical nucleus of the same volume is shown in the Figure. The potential of the ellipsoid is smaller. Thus an increase in nuclear deformation has an effect similar to an increase in volume. In certain isotope pairs, such as ${ }^{86} \mathrm{Sr}-{ }^{88} \mathrm{Sr}{ }^{10}$ and ${ }^{136} \mathrm{Ba}-{ }^{137} \mathrm{Ba}{ }^{11}$ the shift resulting from a g. rease in nuclear deformation with the addition of one neutron is 30 pronounced as to cancel the shift due to the increase in nuclear volume. 


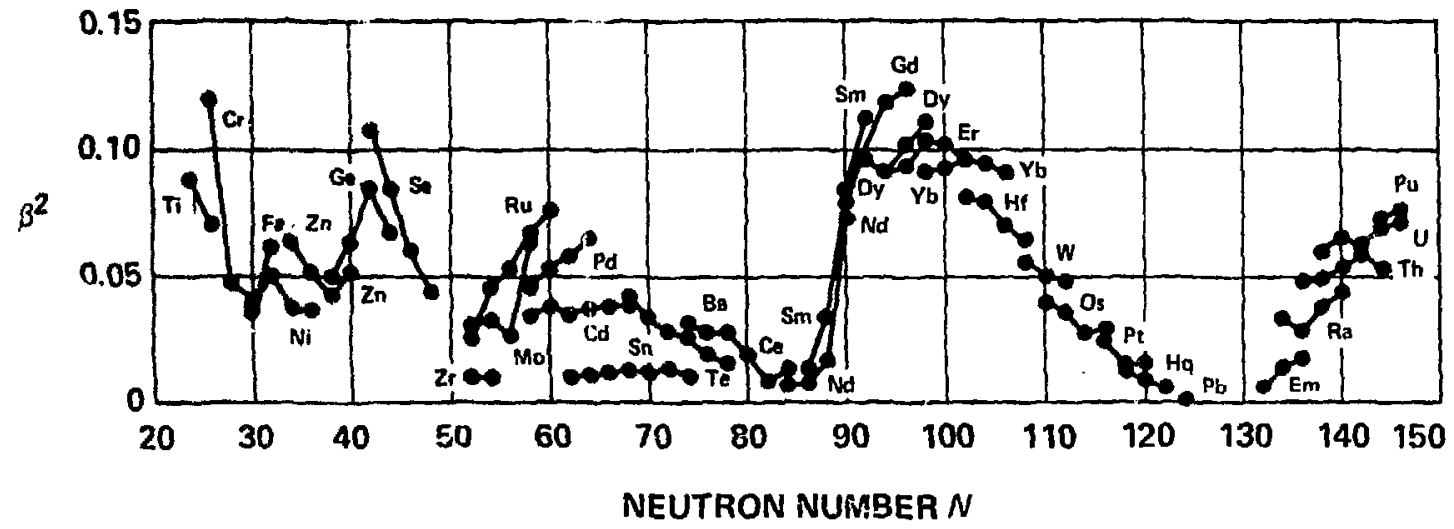

FIGURE 2: The deformation of nuclei from spherical shape as a function of neutron number, $N$. Detormation is defined by the parameter

$e^{2}=\left(\frac{a-b}{r_{0}}\right)^{2}$, where $a$ and $b$ are the semimajor and semiminor axes of the nuclear

ellipsoid of equivalent radius $r$. Only near magic numbers does $\beta^{2}$ approach zero (except for $\mathrm{Sn}$ which has a magic ${ }^{\circ}$ umber $(50)$ of protons). The figure is taken from D. N. Stacey, Reports on Progress in Physics, 29, i7) (1966). 


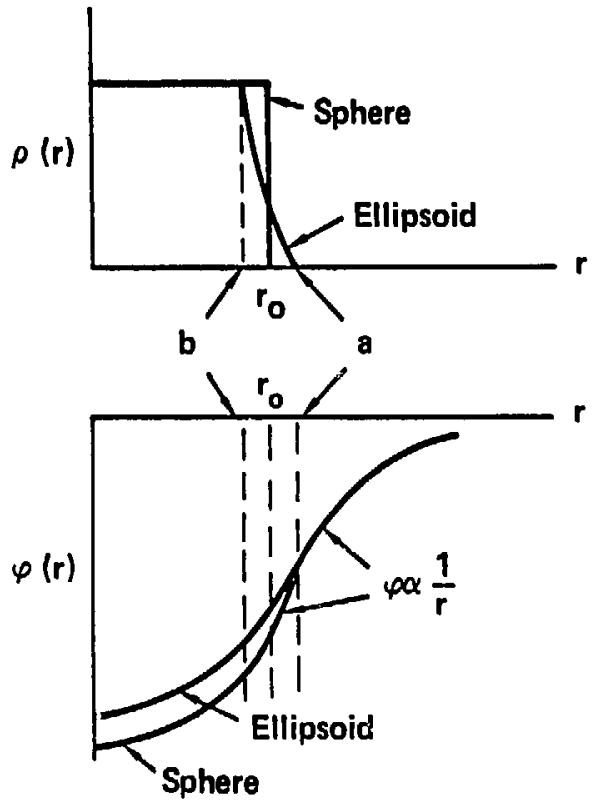

FIGURE 3: a) The charge density, $\rho$, as a function of radius for uniformly charged elliptical nucleus, where charge has been averaged over direction, is compared to that of a sphere of equal volume.

b) The corresponding potentials are compared. It is apparent that an 
The foregoing discussion is intended to give a conceptual understanding of isotope shifts in atoms. For light atoms $(A \lesssim 140)$ the normal mass shift ( $\Delta v \sim \frac{5 \times 10^{-4}}{A} v$ ) is large with respect to the natural linewidth for visible transitions $\left(\Delta v \sim 2 \times 10^{7}\right)$. At the upper end of this range, the two become comparable. For example, the natural linewidth in barium on the $5536 \AA$ transition is $\Delta v \sim 1.9 \times 10^{7} \mathrm{~Hz}$ while the normal mass shift from ${ }^{136} \mathrm{Ba}$ to ${ }^{138} \mathrm{Ba}$ is $3.2 \times 10^{7} \mathrm{~Hz} \cdot{ }^{11}$ on the other hand, the increase in nuclear volume, though partialiy cancelled by a deciease in nuclear deformation, produces a net shift (136-138) of over six times the natural linewidth on the $5536 \AA$ transition. ${ }^{11,12}$ In uranium, the heaviest naturally occurring element, volume and deformation effects combine to produce typical isotope shifts in excess of $3 \times 10^{9} \mathrm{~Hz}$ for most lines in its extremely complex visible spectrum. ${ }^{13}$ It is rare that various effects sum to less than the natural linewidth. Even if the isotope shift on a given transition is small with respect to its natural linewidth, it is generally the case that another line can be found where one of the effects changes magnitude or sign with respect to the others and the resulting isotope shift becomes large.

With an excited state lifetime on the order of $10^{-8} \mathrm{sec}$, an atom can absorb and emit many times during the short interval of time it might take to traverse a laser beam. Under appropriate conditions, the number of absorption-emission events will be sufficient to physically separate the isotopic component of interest from an atomic beam, as illustrated in Figure 4. Acomic vapor is produced inside a container partially filled with the isotopic mixture of interest. The container is heated to bring the atomic vapor pressure of the mixture to its desired value. Atoms issue from a hole in the container and are collimated by an aperture some 


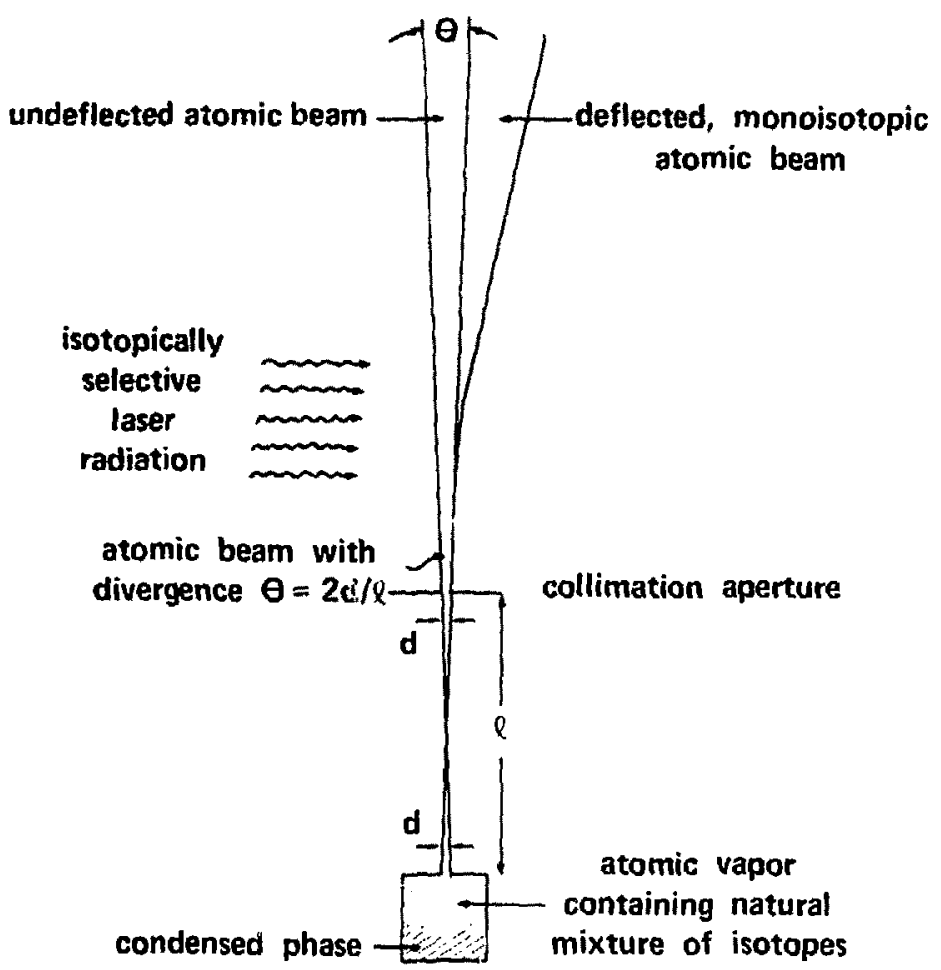

FIGURE 4: Isotope separation by selective absorption of laser radiation by a

single isotopic component of an atomic beam. Atomic vapor containing a mixture of isotopes effuses from a hole of diameter $d$. It is collimated by a similar aperture a distance $a$ away and one isotopic component is deflected by laser radiation in the direction of its propagation. 
distance from the hole. If the gas is collisionless once it leaves the container, it is said to be effusive. Then, the full divergence of the atomic Deam above the collimation aperture is $\theta_{0}=\frac{2 d}{l}$ in the special case where $d$ is the width of both container and collimator apertures. The momentum of an atom perpendicular to the atomic beam axis is $\sin \theta$ times its total momentum where $\theta$ is the angle between the beam axis and the atomic momentun: vector. The amount of momentum which must be imparted the atom from the radiation field in order to deflect it out of the atomic beam is $\frac{N h_{v}}{C}>M v\left(\sin \frac{\theta_{0}}{2}-\sin \theta\right)$. Since $|\theta|<\frac{\theta_{0}}{2}$ and since $\theta_{0}$ can te made very small $\left(10^{-3}\right.$ or less), the number of photons, $N$, which an atom must absorb to be separated can likewise be made to be small. This point will be taken up shortly.

The condition that the gas is collisionless above the hole in the container implies that $\lambda \gtrsim d$ where $\lambda$ is the atomic mean free path inside the container. If $\lambda<d$ then momentum-exchangins collisions occur in the expanding gas above the hole. Since this region of collisional gas is larger in diameter than the hole from which it issued, the atomic beam divergence will be larger than $\frac{2 d}{l}$.

If the condition $\lambda \gtrsim d$ is met, the gas leaving the container is said to be effusive. To show that an effusive yas is effectively collisionless: consider an atom leaving the container. We wish to find the probability, $P$, that the atom will have a collision between some distance, $r_{1}$, above the container and infinity and to show that $P \ll 1$ for $r \ll \lambda\left(r_{1}\right)$. This is equivalent to saying that the gas above the hole becomes collisionless when the local mean free path is large compared to the distance from the hole. The differential collision pronability is given by,

$$
d P=\frac{d r}{\lambda(r)} \text { where } \frac{1}{\lambda(r)}=\frac{n_{0}}{6}\left(\frac{r_{0}}{r}\right)^{2} 0 .
$$


where $\sigma$ is the collision cross-section and the density of the gas at a distance $r$ above the hole is $n=\frac{n_{0}}{6}\left(\frac{r_{0}}{r}\right)^{2} ; n_{0}$ is the density inside the container and $r_{0}$ is the radius of the hole in the container. The collision probability from $r_{1}$ to $\infty$ is

$$
\begin{aligned}
\int_{0}^{P} d P & =\int_{r_{1}}^{\infty} \frac{d r}{(r)}=\int_{r_{1}}^{\infty} \frac{n_{0}}{6} r_{0}^{2} \sigma \frac{d r}{r^{2}} \\
P & =\frac{n_{0}}{6} r_{0}^{2} \frac{\sigma}{r_{1}}=\frac{n_{0} r_{0}^{2}}{6 r_{1}^{2}} \sigma r_{1}=\frac{r_{1}}{\lambda\left(r_{1}\right)}
\end{aligned}
$$

We want $P \ll 1$ so that

$$
1 \gg \frac{r_{1}}{\lambda\left(r_{1}\right)} \text { or } \quad \lambda\left(r_{1}\right) \gg r_{1}
$$

which is the desired result.

This condition when applied to the gas at the hole itself defines a relationship between the diameter of the hole in the container and the density of the gas inside it, $\lambda>d$. This is the effusion condition.

The importance of the divergence of the atomic beam in the plane defined by the laser axis and atomic beam axis is two-fold. First of a11, it is the ?argest angle through which any atom needs to be deflected in order for it to be physically removed from the original atomic beam. Secondly, it determines the Doppler width of the atomic beam with respect to laser radiation incident transverse to the atomic beam axis. The shift in absorption frequency of an atom with velocity component $v \sin \theta$ in the direction of laser propagation, relative to that of an atom with zero velocity component, is 


$$
\Delta v_{D}=\frac{v \sin \theta}{c} v_{0}
$$

where $\nu_{0}$ is the unshifted absorption frequency and $\theta$ is the inclination of the atomic velocity vector with respect to the atomic beam axis. The distribution of atomic velocities in an atomic beam is given by

$$
f(v, \theta)=2\left(\frac{M}{2 k T}\right)^{2} v^{3} e^{-M v^{2} / 2 k T} \cos \theta,
$$

and the distribution of velocities in a given direction perpendicular to the atomic beam axis is

$$
f_{\perp}(v, \theta)=2\left(\frac{M}{2 k T}\right)^{2} v^{3} e^{-M v^{2} / 2 k T} \cos \theta \sin \theta \cos \phi
$$

The average velocity in the beam is found from $f(v, \theta)$ to be ${ }^{14}$

$$
\bar{v}_{b}=\frac{3}{4} \sqrt{\frac{2 \pi k T}{M}}
$$

where $T$ is the temperature of the effusion cnamber. The average velocity in the atomic beani is thus $\frac{3}{4} \sqrt{\pi}$ times the mean scalar velocity in the effusion chamber. The mean scalar velocity perpendicular to the atomic beam is found from $f_{\perp}(v, \theta)$ to be

$$
\bar{v}_{b \perp}=\frac{2}{3} \bar{v}_{b} \sin \frac{\theta}{2}
$$

where $\theta_{0}$ is the beam divergence. The Doppler width of the atomic beam is approximately the difference in absorption frequency between atoms traveling in opposite directions along the laser propagation axis with scalar velocity $\bar{v}_{b \perp}$. 


$$
\overline{\Delta ت}_{D}=\frac{4}{3} \frac{\vec{v}_{b}}{c} \quad v_{0} \sin \frac{\theta}{2} .
$$

The Doppler width of the atomic beam must, first of all, br small compared to the isotope shift, otherwise several isotopic components will be deflected together. The Doppler width must also be small sompared to either the laser linewidth, the natural linewidth, or both. If it is not, an atom can only be deflected through a fraction of the atomic beam divergence angle and separation of a large fraction of the desired isotopic component of the atomic beam will be impossible. It is best if both Doppler and laser linewidth are small compared to the natural linewidth, al though then it makes little difference whether the Doppler linewidth is greater than the laser linewidth or visa versa. The reason this arrangement is preferred is that every atom in the atomic beam then sees the full laser intensity. When the laser linewidth is greater than the natural linewidth only a fraction of the laser radiation falls witrin the absorption range of an atom, that fraction given approximately by $\Delta v_{\text {natural }} / \Delta v_{\text {Taser }}$.

Atomic beam divergences as small as $10^{-3}$ are readily achieved in practice. For example, $d=10^{-2} \mathrm{~cm}$ and $\ell=20 \mathrm{~cm}$ imply such a divergence. The number of absorption-emission events required to effect separation of a given atom from an atomic beam is

$$
N \frac{h v}{c} \gtrsim M v\left(\sin \frac{\theta_{0}}{2}-\sin \theta\right) \approx M v\left(\frac{\theta}{2}-\theta\right)
$$

where the laser beam axis is perpendic, is the atomic beam axis, $v$ is the atomic speed, and $\frac{\theta_{0}}{2}-\theta$ is the $a_{\text {. }}$,.e between the atomic velocity vector and that defining the downwind edre of the atomic beam (the edge. farthest from the laser). 
Consider an "average" atuin in an atomic beam with divergence $\theta_{0}=10^{-3}$. The number of $5500 \AA$ laser photons required to separate it from the original atomic beam is

$$
N_{0}>M \bar{v}_{b} \frac{\theta_{0}}{2} \underset{F i v}{\frac{C}{H}} 2 \times 10^{13} \sqrt{M k T}
$$

$N_{0}$ is largest and separation most difficult for $M$ and $T$ large. This is the case fur a heavy atom if low volatility, where high temperatures are required to produce appreciable vapor pressures. A refractory metal such as uranium $(A \simeq 240)$ must be heated to $2450^{\circ} \mathrm{C}$ before its vapor presslire reaches 1 torr. Using uranium as an example, (tungsten gives almost the identical results) we have $N_{0} \gtrsim 90$. This value for $N_{0}$ is a "worst case" for $\theta_{0} \approx 10^{-3}$.

It remains to demanstrate that a conventional dye laser, operating with a linewidth $\approx 10^{7} \mathrm{~Hz}$, is powerful enough to produce $10^{2}$ successive absorption-emission events in an atom as the atom traverses the laser beam. An argon laser pumped, continuous dye laser such as those described in Section III, delivers about $50 \mathrm{~mW}$ of narrow linewidth tunable optical power. Operating at $5500 \AA$ over a $1 \mathrm{~cm}^{2}$ area this implies a flux of $1.2 \times 10^{17}$ photons $/ \mathrm{cm}^{2} \mathrm{sec}$. The rate at which an atom will absorb is given by the photon flux times the absorption cross section. The absorption cross section at line center is given by

$$
\sigma=\frac{g_{2}}{g_{1}} 4 \pi x^{2},
$$

where $g_{2}$ and $g_{1}$ are the degeneracies of upper and lower states respectively so that for a $5500 \AA$ transition

$$
4.4 \times 10^{-10} \therefore \sigma \leqslant 4 \times 10^{-9} \text {. }
$$


Thus the absorption rate becomes

$$
5 \times 10^{7} \lesssim R 乞 4.8 \times 10^{8} \text { photons } / \text { sec. }
$$

For simplicity, let $R=10^{8}$. Then the average time for one absorption and one emission to take place with $\tau=10^{-8} \mathrm{sec}$ is

$$
\frac{1}{R}+\tau \sim 2 \times 10^{-8} \mathrm{sec}
$$

and the rate at which the absorption-emission cycle is repeated is $5 \times 10^{7} / \mathrm{sec}$. Comparison of the spontaneous decay rate $\left(\frac{l}{\tau}\right)$ with the absorption rate $(R)$ shows that much greater laser intensities serve little purpose since they only increase the abscrption rate whereas spontaneous decay is the rate limiting process. One hundred absorption-emission events will take place in a time of $2 \times 10^{-6}$ seconds, or the time it takes an atom to travel a distance $\delta=2 \times 10^{-6} \mathrm{v} \mathrm{cm}$. For atomic vapors at 1 torr,

$$
2 \times 10^{4} \lesssim \bar{v} \lesssim 3 \times 10^{5} \mathrm{~cm} / \mathrm{sec}
$$

The lower limit is set by mercury at $273^{\circ} \mathrm{C}$. Mercury and noble gases have vaper pressures in excess of 1 torr at room temperature. For such elements, gas pressure in a reservoir can be controlled directly by restricting flow into the reservoir from a higher pressure source so that it is equal to the rate of effusive flow out of it. The higher limit is set by boron and carbon which are at the same time light and refractory. Thus

$$
0.036 \mathrm{~cm} \lesssim \delta \lesssim 0.54 \mathrm{~cm}
$$


The discussion which led to an absorption rate of about $10^{8}$ photons/sec assumed that the $50 \mathrm{riw}$ dye laser beam was expanded to a cross section of $1 \mathrm{~cm}^{2}$. Since $\delta \leq 0.54 \mathrm{~cm}$, iaser beam which irradiates the atomic beam along height $h n 0.54 \mathrm{~cm}$ parallel to the atomic beam axis is sufficient to separate an "average atom" of the desired isotope. The width of laser and atomic beams can be $1.85 \mathrm{~cm}$ while the thickness of the atomic beam along the laser axis is determined from the divergence condition. Thus a commercial dye laser has sufficient intensity to separate isotopes on a laboratory scale.

The principal limitation on the number of absorption-emission events per atom is the accessibility of metastable energy levels between the optically excited state and the ground state. Lifetimes of such states are many orders of magnitude longer than states whose radiative decay to the ground state is fully allowed. ${ }^{16}$ When an excited atom decays to such a state, it can no longer absorb laser photons and therefore cannot be further deflected. Suppose, for example, that the excited state has a probability $P_{1}$ of decaying to a metastable state and a probability $P_{0}$ of returning to the ground state. Then the probability that an atom absorbs and returns to the ground state $\mathrm{N}$ times before decaying to the metastable state after the $\mathrm{N}+7^{\text {st }}$ absorption is

$$
P_{1} P_{0}^{N}
$$

Suppose that an "average atom" requires $N_{0}$ photons for separation. Then the fraction of atoms of the desired isotope in the beam that will be separated, is approximately 


$$
\begin{aligned}
E & \sim P_{1} \int_{N_{0}}^{\infty} P_{0}^{N} d N \text { for large } N \\
& =P_{1} \int_{N_{0}}^{\infty} e^{N \ln P_{0}} d N=\frac{P_{1}}{-\ln P_{0}} e^{N_{0} \ln P_{0}} \\
& \simeq e^{N_{0} \ln P_{0}}
\end{aligned}
$$

(see section V. F.). Suppose that $N_{0} \approx 10^{2}$. Then $\varepsilon=\frac{1}{e}$ for $N_{0} \ln P_{0}=-1$ and $P_{1} \sim-\ln P_{0} \sim 10^{-2}$. Thus, if an average atom has to absorb (and re-emit) 100 photons in order to be separated, less than 1/e of the desired atoms in the beam will be separated if the probability of decay to a metastable state is more than $1 \%$. This example illustrates the deleterious effect that even a small probability of decay into a metastable state has on the efficiency of the isotope separation. If it is not possible to avoid or tolerate metastable state accumulation, high efficiency separation can be accomplished with the use of additional lasers to excite metastable atoms to a state (e.g. the original excited state) from which they can again decay to the ground state. Only when metastable states with non-negligible accumulation rates are too numerous or the depopulating transition occurs at a frequency for which no convenient photon source exists, does efficient photodeflection become impractical.

Another limitation might be expected to arise when the density of the atomic beam in the region in which deflection takes place is too high. Induced palarization effects cause small angle atom-atom scattering. As a result of such scattering, small atomic beam divergences cannot be maintained at high beam densities. Consider the Van der Waals interaction 
between one atom and another passing close by it. Let $v$ be their relative velocity and let $b$ be the distance of closest approach in the absence of any interaction ( $b$ is called the impact parameter). If the gas is not toc dense, $b$ will generally be larje enough so that the Van der Waals interaction, $U$, is weak and the angles of deflection are small. In this approximation the momentum transferred to the passing atom perpendicular to its original direction of travel is

$$
P_{\perp}=\int_{-\infty}^{\infty} F_{\perp} d t=-\frac{2 b}{v} \int_{b}^{\infty} \frac{d u}{d r} \frac{d r}{\sqrt{r^{2}-b^{2}}}
$$

where $r$ is the distance between the atoms. ${ }^{17}$ The result for a $U=\frac{a}{r^{n}}$ potential is 17

$$
P_{\perp}=\frac{2 a \sqrt{\pi}}{v b^{n}} \frac{\Gamma\left(\frac{1}{2} n+\frac{1}{2}\right)}{\Gamma\left(\frac{1}{2} n\right)}
$$

This kind of scattering begins to have significant effect on photodefiection isotope separation when

$$
P_{\perp} \sim N_{0} \frac{h v}{c}
$$

that is, when atoms are scattered as far as they are deflected by radiation.

The exact expression for the Van der Waals interaction is now derived. ${ }^{18}$ The interaction of both ground and excited atoms of an isotope a with a ground state atom of a different isotope $b$ will be considered. The unperturbed wavefunctions for the system are taken to be

$$
\begin{array}{ll}
\psi_{00}=\phi_{a 0} \phi_{b 0}, & \psi_{10}=\phi_{a 1} \phi_{b 0}, \\
\psi_{01}=\phi_{a 0} \phi_{b 1}, & \psi_{11}=\phi_{a 1} \phi_{b i}, \text { etc. }
\end{array}
$$


The perturbation in the Hamiltonian of alkali-like atoms is given by

$$
v=-\frac{e^{2}}{r_{B a}}-\frac{e^{2}}{r_{A b}}+\frac{e^{2}}{r_{A B}}+\frac{e^{2}}{r_{a b}}
$$

where $r_{B a}$ is the distance between the nucleus of isotope $b$ and the electron of isotope $a, r_{A b}$ is the distance between the nucleus of isotope a and the electron of isotope $b$, and so on. The perturbation Hamiltonian can be expanded into dipole-dipole interaction, dipole-quadrupole interaction, quadrupole-quadrupole interaction, etc. Keeping only the dipole-dipole part, the perturbation becomes

$$
v_{d}=\frac{e^{2}}{R^{3}}\left(x_{a} x_{b}+y_{a} y_{b}-2 z_{a} z_{b}\right)
$$

where $R=r_{A B}$ and $x_{a}, x_{b}, z_{a}$ are the coordinates of the electron. The first order perturbation in the energy is zero:

$$
E_{1}=\sim_{\mathbf{i j}}\left|V_{\mathbf{d}}\right| \psi_{\mathbf{i j}} \cdot=0
$$

The second order perturbation in the energy is

$$
E_{2}=\sum_{k, l} \frac{-\psi_{i j}\left|V_{d}\right| \psi_{k \ell} \psi_{k \ell}\left|V_{d}\right| \psi_{i j}{ }^{3}}{E_{i j}-E_{k l}}
$$

Thus, for example, the energy of interaction of ground state atoms is

$$
E_{2}=\frac{\left[\psi_{00}\left|v_{d}\right| \psi_{11}>\left\langle\psi_{11}\left|v_{d}\right| \psi_{00}\right\rangle\right.}{2 h{ }_{10}}+\cdots \cdot
$$

This leads to the expression for the Van der Waals interaction given by London: 19 


$$
u=-\frac{3}{4} \frac{2}{r^{6}} i
$$

where, is the atomic polarizability. I is the ionization potential and $r$ is the distance between the interacting atoms. For one isotope excited and one in the ground state we have

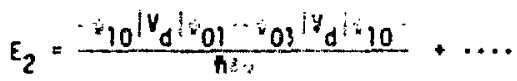

where now the denoninator is $h$ times the isotope shift. Thas, because the energy of the system in which isotope a is excited while isotope $b$ is in the ground state, is almost identical to the system in which isotope b is excited and isotope a is in the ground state, the perturbation is larger by about a factor of $2 v / 5:$.

The expression for transverse momentum imparted by the scattering process now becomes

$$
p_{1} 2 \frac{4 \sqrt{\pi}}{3} \frac{e^{4} \vec{r}_{a}^{2} \vec{r}_{b}^{2}}{v b^{6 h \delta v}} \frac{r\left(\frac{7}{2}\right)}{r(3)}
$$

where $\bar{r}_{\mathrm{a}}=\left.i_{\mathrm{a}_{0}}\left|\mathbf{r}_{\mathrm{a}}\right|_{\mathrm{a}} \cdot\right|^{2}$

or

$$
P_{1}=\frac{3}{2} \sqrt{\pi} \frac{a^{2} !}{v b^{6}} \frac{r\left(\frac{7}{2}\right)}{r(3)} \frac{2 v}{8 v}
$$

For most atoms $10^{5} \approx \frac{2 v}{6 v} \approx 10^{8}$ so that the increase in scattering angle resulting from excitation of one isotopic species is impressive. The condition $P_{\perp} \ll N_{0} \frac{h v}{c}$ gives an upper limit for the density of atoms in the atcmic beam, namely 


$$
n^{2} \therefore N_{0} \frac{h v}{I} \frac{v}{c} \frac{5 v}{v} \frac{1}{5 \sqrt{\pi} \alpha^{2}} \text { atoms } / \mathrm{cm}^{3}
$$

With $\frac{h v}{I}=\frac{1}{5}, \frac{v}{c} v 10^{-6}, \frac{s v}{v} \sim 10^{-8}$ and $a \approx 3 \times 10^{-23}$, the above condition becomes

$$
n \cdot 5 \times 10^{14} \cdot \sqrt{N_{0}} \text { atoms } / \mathrm{cm}^{3} \text {. }
$$

This is as stringent a restriction on the density as is likely to be encountered.

The density of atoms at the source of the atomic beam is given by the relation

$$
n=\left(\frac{r_{0}}{r}\right)^{2} \frac{n_{0}}{6}
$$

where $n$ is the density of atoms a distance $r$ from the source aperture whose radius is $r_{0}$. Thus, the source density is governed by the inequality

$$
n_{0}<6\left(\frac{r}{r_{0}}\right)^{2} n_{\max }=\frac{6 n_{\max }}{\left(\frac{\theta}{2}\right)^{2}}
$$

where ${ }_{0}$ is the divergence of an atomic beam defined by identical source and collimater holes of radius $r_{0}$. With $\theta_{0} \approx 10^{-2}$ and $n_{\max } \approx 5 \times 10^{14} \sqrt{N_{0}}$, the source density cannot exceed $1.2 \times 10^{20} \sqrt{N_{0}}$ atoms $/ \mathrm{cm}^{3}$.

From this example it can be seen that Van der Wals scattering poses no significant restriction on the parameters of a photodeflection isotope separation process.

Another process which one might expect to limit efficiency and produce fractional enrichment is excitation exchange between different isotopes. The density at which excitation exchange becomes likely is given approximately by

$$
\frac{\mathrm{E}_{2}}{\mathrm{~h} \delta \mathrm{v}} \because 1
$$


where $E_{2}$ is the second order perturbation of the energy of a system containing one excited atom of isotope a and one ground state atom of isotope $b$ and $\delta v$ is the isotope shift on the corresponding transition. Thus excitation exchange is likely for

$$
\frac{e^{4}}{R^{6}} \frac{\left|r_{0}\right|^{4}}{\left(h_{\delta}\right)^{2}} \sim \frac{3}{2} \frac{\alpha^{2}}{R^{6}} \frac{I}{h_{v}}\left(\frac{v}{\delta v}\right)^{2} \sim 1
$$

or

$$
n^{2} \propto \frac{2}{3 \alpha^{2}} \frac{h \cdot v}{I}\left(\frac{\delta v}{v}\right)^{2}
$$

Using the same numbers as were used in the scattering example, the most stringent limitation which excitation exchange might place on density is $n<10^{14} / \mathrm{cm}^{3}$. Since photodeflection relies on momentum transfer from many absorption emission events, however, excitation transfer has essentially no effect. Excitation exchange is equivalent to emission by an atom of the desired isotope and absorption by an adjacent atom. It therefore does not affect the deflection of the desired isotope which depends on absorption alone. An atom of the unwanted isotope can gain momentum in the process, but the direction of momentum change is random. Furthermore, a single unit of momentum $\frac{h_{v}}{c}$ is far from sufficient to produce separation. If $n$ units of momentum are required to deflect an average atom out of the beam, then, because of the isotropic nature of the excitation transfer process, an atom of unwanted isotope would have to take part in an average of $n^{2}$ excitation transfers to be separated. Thus only very small contamination could result even if the density of the atomic beam were high enough to make excitation transfer a significant 
process. Only in a separation process which relies on energy transfe, from the radiation field, $20,21,22$ dees excitation exchange pose a Iimitation on atom density. 


\section{PHOTODEFLECTION APPLIED TO BARIUM ISOTOPES}

Barium is element number 56 on the periodic table of the elements. It has seven stable isotopes which are listed in Table 3 along with their natuial abundances. The heaviest isotope, ${ }^{138} \mathrm{Ba}$, is also the most abundant. In heavier elements of even 2 , isotopic abundances are usually largest for moderately heavy isotopes, smaller for the heaviest isotope and smallest for the lightest isotope. The unusua? ${ }^{i 38_{B a}}$ abundance is a result of the fact that ${ }^{138} \mathrm{Ba}$ has 82 neutrons, one of the magic numbers of nuclear structure, analogous to noble gas electronic structure, and very stable.

Barium is an alkali earth element. In its ground state barium has two 6 s electrons outside of a closed shell xenon electronic structure. An en gy level diagram of low lying singlet states is given for reference in Figure 5. The barium resonance line, $6 s^{2}{ }^{1} S_{0}-6 s 6 p{ }^{1} p_{1}$, occurs at a wavelength of $5535.7 \AA$. This transition gives a flame containing barium its characteristic green color. Since a number of dyes lase efficiently at $5535.7 \AA$, the barium resonance could be used to demonstrate photodeflection isotope separation.

Barium is a metallic solid at room temperature. It melts at $725^{\circ} \mathrm{C}$ and boils at $1638^{\circ} \mathrm{C}$. The 1 iquid has a vapor pressure of 1 torr at $800^{\circ} \mathrm{C}$. An atomic beam effusing from an $800^{\circ} \mathrm{C}$ source would have an average velocity of

$$
\bar{v}_{b}=\frac{3}{4} \sqrt{\frac{2 \pi k T}{M}} \sim 6 \times 10^{4} \mathrm{~cm} / \mathrm{sec}
$$

which implies that the number of absorption-emission events required to separate an "average" atom from an atomic beam of divergence $\theta_{0}$ is 


\section{TABLE 3}

Isotope

130

132

134

135

136

137

138
Natural Abundance

0.10

0.10

2.42

6.59

7.81

11.32

71.66 


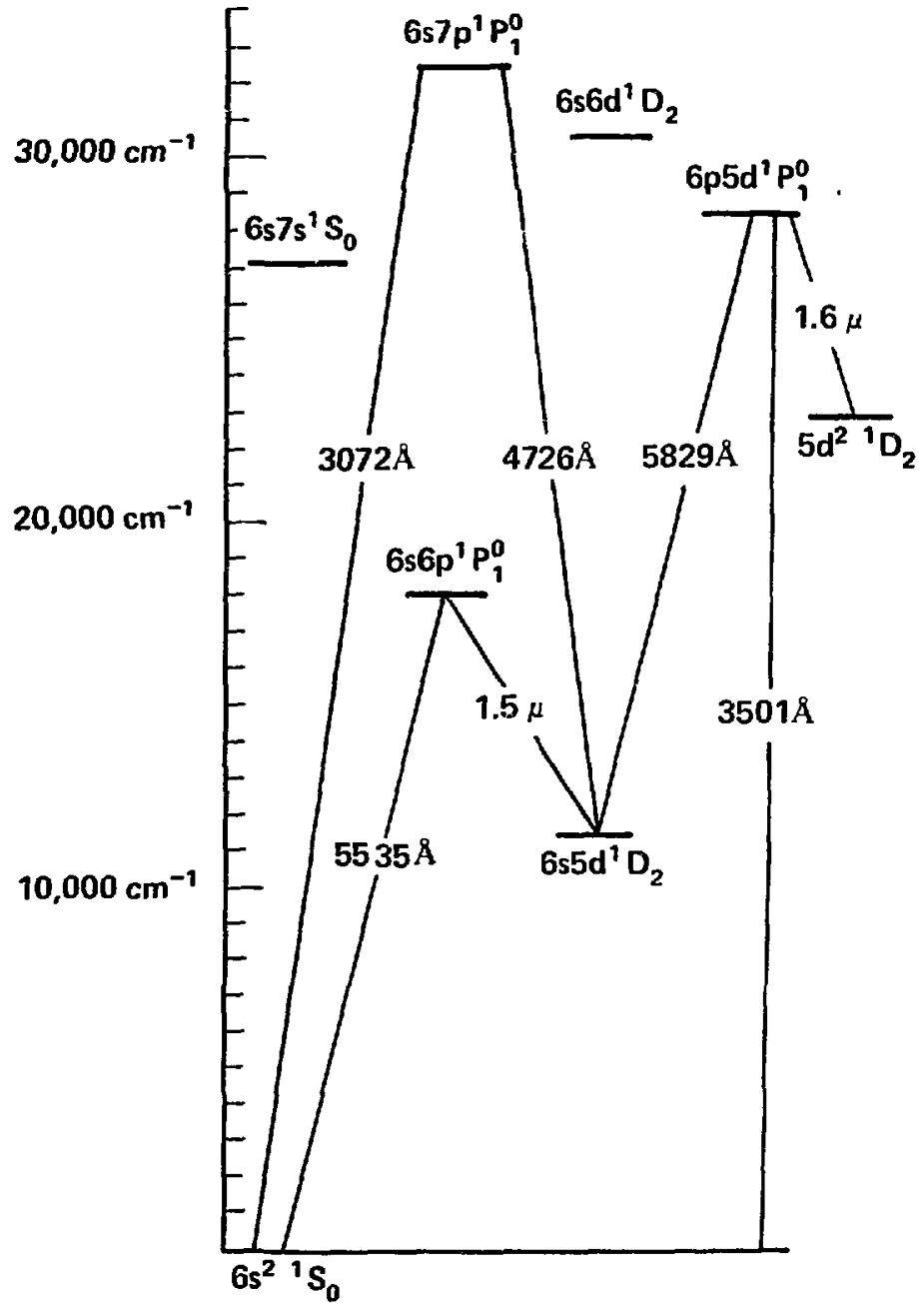

FIGURE 5: Low lying singlet energy leve1s and selected transitions in $\mathrm{Ba} \mathrm{I}$. 


$$
n_{0} \approx M \bar{v}_{b} \frac{\theta_{0}}{2} \frac{c}{h_{v}} \approx 4 \times 10^{4} \theta_{0}
$$

The lifetime of the upper $6 s 6 p P_{1}$ state is $8.37 \times 10^{-9} \mathrm{sec}^{23,24}$ which implies a $19 \mathrm{MHz}$ natural linewidth. It has been noted that both laser and Doppler linewidth should be small compared with the natural Iinewidth. This is not difficult, since laser lintwidths of less than $10 \mathrm{MHz}$ are readily achieved, while a Doppler width smal. compared to 19 MHz implies

$$
\begin{aligned}
& \Delta v_{D} \sim \frac{4}{3} \frac{\bar{v}_{b}}{c} \sin \frac{\theta_{0}}{2} \nu_{0} \approx 8 \times 10^{8} \theta_{0} \ll 1.9 \times 10^{7} \mathrm{MHz} \\
& \theta_{0} \ll 2.4 \times 10^{-2} \mathrm{rad}
\end{aligned}
$$

In barium, a single metastable state is accessible from the $6 s 6 \mathrm{p}{ }^{\prime} p_{1}$ level. This is the $6 s 5 d d^{1} D_{2}$ state from which decay to the grourd state is parity forbidden, being allowed only via an electric quadrupole transition. Since such a transition has a lifetime rougiily $\left(\mathrm{ka}_{0}\right)^{-2}$ times the corresponding dipole lifetime, ${ }^{25}$ the ${ }^{1} \mathrm{D}_{2}$ state will have a lifetime given approximately by $\tau^{-1} \leadsto 0.5 \mathrm{~s} .^{26}$ Thus an excited barium acom in the atomic beam which decays to the ${ }^{D_{2}}$ state will not return to the ground state to undergo further deflection before it has, on the average, traveled $230 \mathrm{C}$ meters.

The probability that an excited atom Lecays into the metastable state once excited, relative to the probability that it decay's to the ground state, is found from the ratio of spontaneo:is decay rates for the two transitions. Recalling the expression for the decay rate to a single siate, 


$$
\tau_{12}^{-1}=\frac{8 \pi e^{2}}{m_{e}^{c}} \cdot \frac{f_{12}}{x_{12}^{2}} \frac{g_{2}}{g_{1}},
$$

the ratio of spontaneous decay rates for the $6 s 6 p p_{1}$ level in bariun is found, using published values of the oscillator strengths, ${ }^{25}$ to be

$$
\frac{g_{s}}{g_{d}} \frac{f_{s}}{f_{d}} \frac{\left(\lambda_{d}\right)^{2}}{\left(\lambda_{s}\right)^{2}}=\frac{1}{5} \frac{1.59}{0.10} \frac{(15000)^{2}}{(5535)^{2}} \simeq 24
$$

Thus, for a given atom in the $6 s 6 p{ }_{p}$ state, the probability that it radiates into the $I_{D}$ state is $\frac{1}{25}$. The probability that a given atom scatters $n$ photons befrre being pumped into the metastable level is $\left(\frac{24}{25}\right)^{n} \frac{1}{25}=\frac{e^{-0.04 n}}{25}$ and the separation efficiency is approximately

$$
\epsilon \sim e^{-0.04 \times 4 \times 10^{4} \theta_{0}} \sim e^{-1.6 \times 10^{3} \theta_{0}}
$$

The actual efficiency was found to be much greater than this expression impiies. The error in the "average atom" approximation is nnt large. Rather, the published experimental vaiue of the $6 s 6 \mathrm{p}{ }_{F_{1}}$ $6 s 5 d{ }^{1} D_{2}$ oscillator strength was found to be high so that decay to the metastable state from the $6 s 6 p P_{P_{1}}$ level was less probable than expected. The reader isreferred to Section V.F. for details. In barium, as in most other atoms, several avenues of metastable state depopulation are available. An atom in the $6 s_{5 d}{ }^{1} D$ state can be excited to several levels from which it can decay to the ground state. If a laser were readily available at $1.5 \mu$ wavelength, the wisest course of ${ }^{1} D_{2}$ level destruction would be to excite tine metastable atom back to the $6 s \sigma_{p} I_{p_{1}}$ state. Since no powerfut laser is available at this wavelength, either the $5826 \AA$ transition to the $5 d 6 p \quad P_{P_{1}}$ state or the $4726 \AA$ transition to the $6 s 7 p P_{P_{1}}$ 
state could be chosen for metastable state depopulation. These states have nearly equal oscillator strength for decay to the ground state $27,28,29$ indicating that their wavefunctions are thoroughly mixed. (0therwise, the $6 \mathrm{p} 5 \mathrm{~d}$ state would be metastable.) The $5826 \AA$ ine was chosen in this work for the practical reason that much more power is available at this wavelength than at 4726: blue dyes require UV pumping and powerful enough continuous UV ion lasers were not available at the time this thesis was undertaken. Ir. addition blue dyes have less gain and lower lasing efficiency than the visibie dye actually used in the experiments. The disadvantage of depopulation via either $6 s 7 p$ or $6 p 5 d$ states is the accessibility of another metastable state, the $5 \mathrm{~d}^{2} \mathrm{l}_{\mathrm{D}_{2}}$ state at $23062 \mathrm{~cm}^{-1} .30$ The oscillator strength for decay to this state was not known at the outset of this work but has been measured as part of it (see Section V.G.).

Barium isotopes exhibit unusually small isotope shifts. The positions of isotopic absorption peaks on the $5536 \AA$ transition reported by previous investigators are summarized in Figure 6. The absorption spectrum of natural barium has nine components (excluding the rare ${ }^{130} \mathrm{Ba}$ and ${ }^{132} \mathrm{Ba}$ isotopes), one component for each of the even isotopes 134,136 , and 138 , and three for each of the odd isotopes 135 and 137 (nuclear spin 3/2). The most recent data, that of Jackson and Duong, is based on absorption spectra of enriched samples in an atomic beam and is quite accurate. This is the only experiment in which the hyperfine structure of the odd isotopes of barium were resolved. Previous investigators who used enriched samples had to infer the positions of the hyperfine components from the centroid and width of the hollow cathode tube emission curve representing the combined hyperfine peaks. Thus, Jackson and Duong, and Mack assumed that a transition involving a higher $F$ state of excited barium occurred at 
lower frequency than one involving a lower $F$ state while Jackson and Arroe assumed the opposite order. Kopfermann and Wessel assumed that the hyperfine structure was sma11 compared to the isotope shift.

From the recent data of Jackson and Duong it would appear that most, if not all, isotopic absorptions of barium are spaced by more than the natural linewidth of $19 \mathrm{MHz}$ for the $5535.7 \AA$ transition. Inasmuch as isotope shifts in barium are among the smallest measured, barium was regarded as an unusually challenging test of the photodeflection method of isotope separation. 


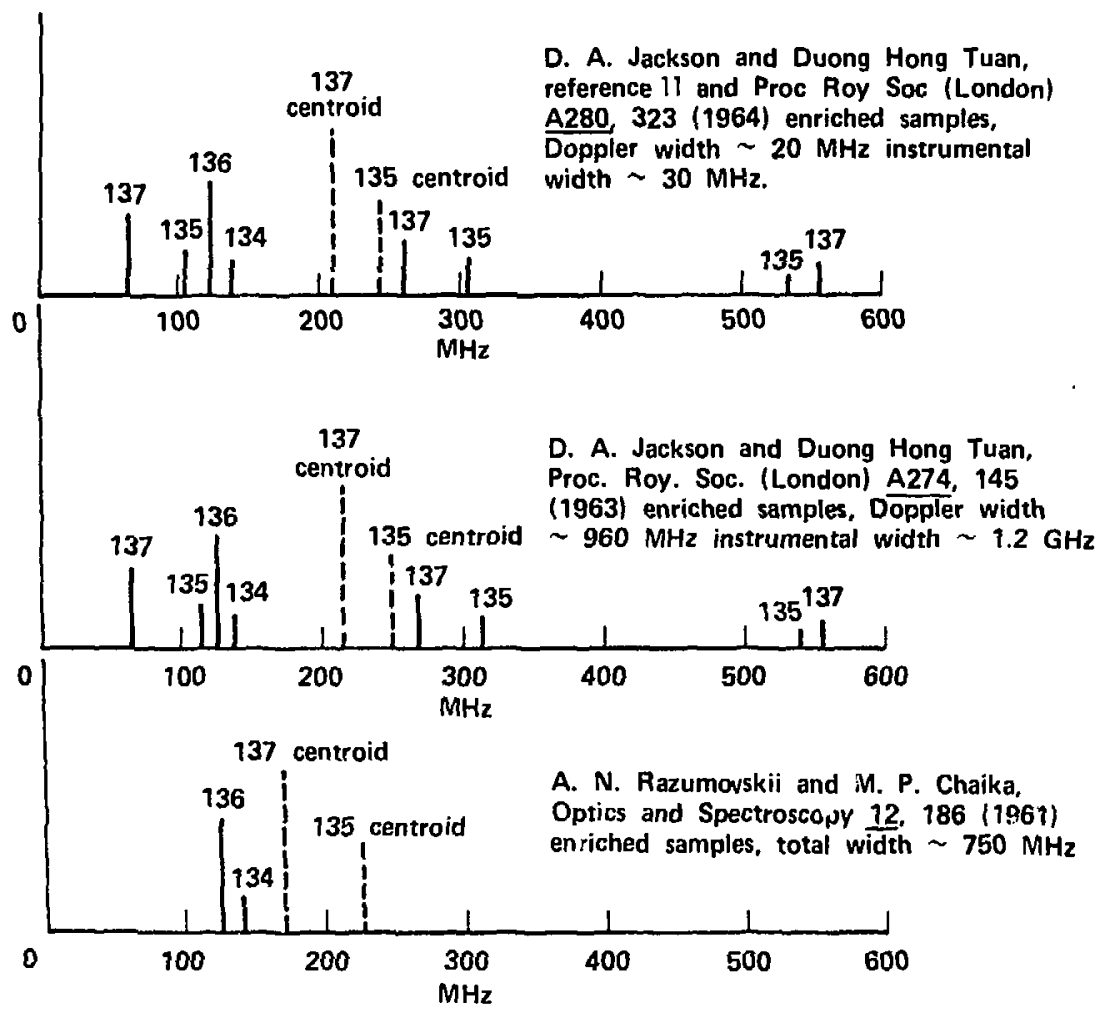

FIGURE 6: Isotopic hyperfind structure of the Ba $5536 \AA$ resonance as determined by previous investigators. 


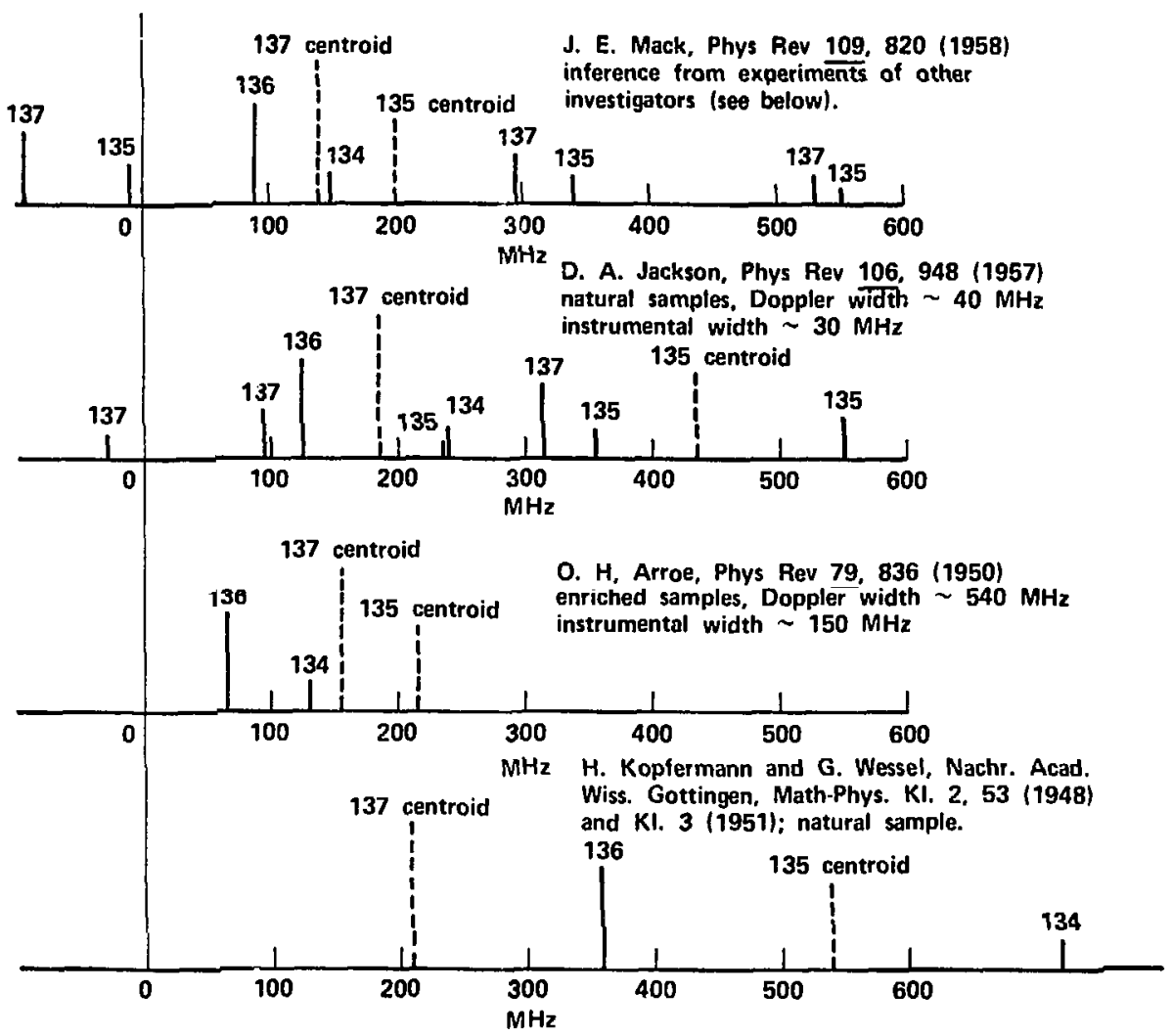

FIGURE 6 (Continued) 


\section{DYE LASERS AND OPTICAL SPECTRUM ANALYZERS}

The lasers used in the research reported here are optically pumped organic dye lasers. They employ rhodamine $6 G$ as their active ingredient. Rhodamine $6 G$ dissolved in water (with soap), methanol, ethanol, propanol, or ethylene glycol lases between about $5600 \AA$, and $6300 \AA$. Dissolved in a $3 / 1$ mixture water/hexafluoro-2-propanol/HFlP), it lases between about $5400 \AA$ and $6000 \AA$. Laser power as a function of frequency is given in Figure 8 for the $\mathrm{H}_{2} \mathrm{O} / \mathrm{HF} \mathrm{P}$ and ethylene glycol solutions. The HFlP shifts the fluorescence of the rhodamine towards the blue by reducing the polar nature of the solvent. In a completely non-polar solvent the fluorescence spectrum of the molecule tends to be identical to the absorption spectrum, as it is in the vapor phase. In polar solvent the molecule acquires a cage of solvent molecules. The dipole-dipole interaction of solute and solvent lowers the energy of the rhodamine in both excited and ground states. When the molecule radiates, however, the solvation shell does not have time to change and the lower energy level differs from its least energy configuration in that it still has the solvation shell characteristic of the excited dye molecule. Similarly, when the molecule absorbs radiation, the lower state is a minimum but the upper level isn't because it retains the hydration layer of the lower state during the absorption process. Thus, the polar solvent red shifts the fluorescence spectrum and blue shifts the absorption spectrum. Neutralizing the polar water solvent with HFIP therefore has the effect of shifting the fluorescence back toward the blue.

It is seen from the Figure 7 that the Rh6G in $\mathrm{H}_{2}{ }^{0 / H F I P}$ has peak fluorescent amplitude near the $5536 \AA$ green resonance of barium, which is why this dye solution was employed. The $5826 \AA^{\circ} \mathrm{O}_{2} \rightarrow P_{1}$ line of barium 


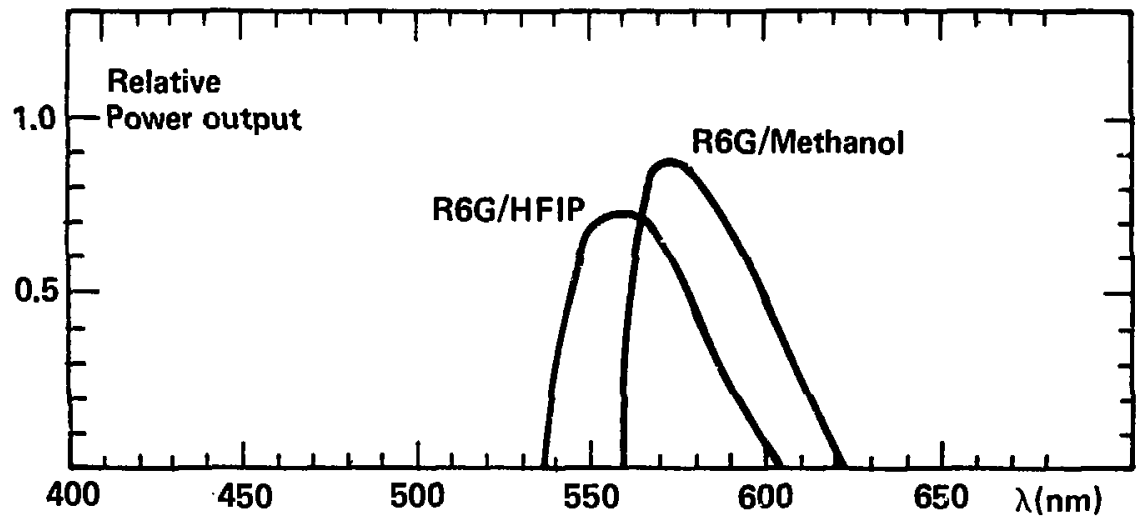

FIGURE 7: Relative lasing power as a function of frequency for Rhodamine 6G dissolved in a 3:1 mixture of water and hexafluoro-2-propanol and Rhodamine $6 G$ dissolved in methanol (or other polar solvent). 
lies in the Rh6G in polar solvent fluorescence spectrum and the second laser used to depopulate the ${ }^{1} D_{2}$ state employed $R 66 G$ in ethylene glycol.

The bandwidth over which a dye laser operates can be made very small, as little as $10^{5} \mathrm{~Hz}^{31,32}$ by the introduction of optical elements into the laser cavity. Furthermore, a large fraction of the unrestricted broadband laser power can be extracted in narrow linewidth operation. The reason for this is as follows. Rotational levels of the dye molecule are spaced by a hundred wave numbers or less. Far from being well defined, energy states are perturbed by electrostatic and collisional interaction with solvent molecules so that individual vibration-rotation levels cannot be distinguished in solution by separate absorption or emission lines. Since only a few collisions times ( $t_{\text {collision }} \approx 10^{-12} \mathrm{sec}^{33}$ ) are required to establish rotation-vibration equilibrium, a typical excited electronic state with a $5 \times 10^{-9} \mathrm{sec}$ lifetime ${ }^{34}$ can come into vibrational-rotational equilibrium before it radiates. In equilibrium, only states within a few $k T$ of the lowest vibration-rotation level (fundamental) of a given electronic level will be populated. Thus, emission generally occurs from the fundamental level of the excited electronic state and absorption occurs from the fundamental level of the ground electronic state. In a dye laser, rapid equilibrium is responsible for rapid repopulation of the fundamental level of the excited electronic state and rapid depopulatior of the excited vibration-rotation level of the ground electronic state which is the lower laser level. For this reason, optical elements can be introduced into the laser cavity which prevent lasing except in a narrow frequency band and excited molecules can be forced to lase in this band. 
The $5536 \AA$ laser radiation was produced by a Spectra Physics model $370 / 581$ dye laser which employed a $2 \times 10^{-4} \mathrm{M}$ solution of Rh6G is a $3: 1$ mixture of water and HFIP. The laser gave $50 \mathrm{mH}$ of output power in a $6 \times 10^{-5} \AA(6 \mathrm{MHz})$ wide band. It was pumped by a $1.6 \mathrm{~W}$ Argon laser operating on all lines. The optical design of the Spectra Physics dye laser is given in Figure $8 .^{35}$ The output of an argon laser, operating on its fundamental (TEM ${ }_{00}$ ) transverse mode ${ }^{36}$ is focused to a small spot just inside the active dye medium. The spot size of an argon laser beam is the same as the spot size of the fundamental TEM ${ }_{00}$ transverse mode of the dye laser cavity providing efficient lasing in this mode and suppressing lasing in other modes which have larger spot sizes. 36

A prism and an etalon have been added inside the cavity to further restrict lasing to a single longitudinal mode of the laser cavity. Such modes are defined by the condition that an integral number of half wavelengths must just fit in the cavity. Thus $\frac{n \lambda}{2}=l_{c}$ where $n$ is an integer and $\ell_{c}$ is the distance between the cavity end mirrors. The difference in wavelength between adjacent modes is $\frac{\lambda}{n}=\frac{\lambda^{2}}{2 l_{c}}$. Since $\ell_{c} \sim 30 \mathrm{~cm}$ or the Spectra Physics laser and $\lambda=5536 \AA$, modes are spaced by about $5 \times 10^{-3} \AA$. The prism restricts lasing to 200 or so longitudinal modes by deflecting all photons but those in a $1 \AA$ wide band out of the cavity. The resolving power relation for a prism is given by $37 \frac{d \phi}{d \lambda}=\frac{d \phi}{d n} \frac{d n}{d \lambda}$ where $d \phi$ is the change in the angle through which a light beam is bent when its wavelength is changed by $d \lambda$. The index of refraction of the prism, $n(\lambda)$, and its derivative $d n / d \lambda$ are properties of the constituent material (e.g. flint glass) and $\frac{d \phi}{d n}$ depends on the geometry of the prism and the size of the light beam. ${ }^{37}$ 
To Prism Amplifier

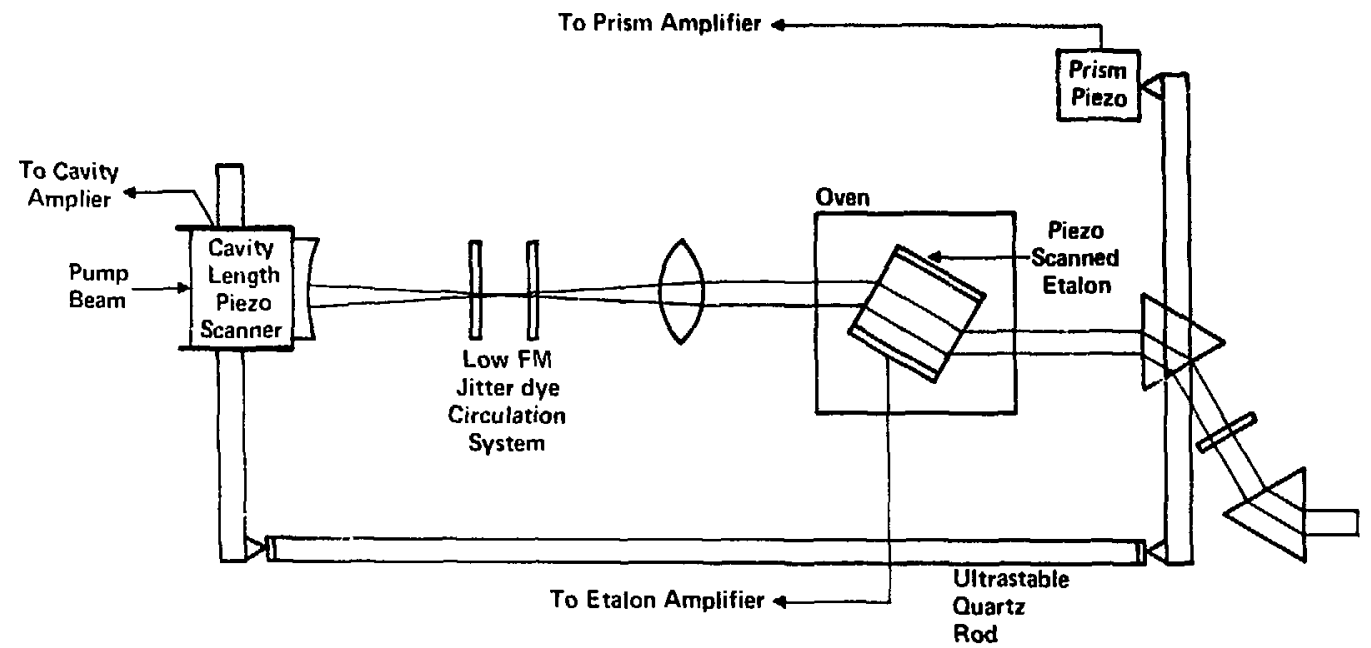

FIGURE 8: Optical design of Spectra Physics Model 370/581 dye laser. 
The etalon produces further frequency discrimination by introducing a second periodic condition, namely, $m \frac{\lambda}{2}={ }^{\ell} e$, where $m$ is an integer and $\ell_{e}$ is the distance between the reflecting surfaces of the etalon. The periodic condition introduced by the etalon is not absolute since the distance between the reflecting surfaces is not exactly uniform across even small parts of their areas. Also, the surfaces are not perfectly reflecting. The ratio of the etalon cavity mode spacing (knowr. as its "free spectra range") to its band pass is known as the etalon finesse. Commercial etalons are available with finesse as high as 200 but these are not used in laser cavities because their transmission is relatively poor. ${ }^{38}$ The finesse of the Spectra Physics etalon is 30 while its free spectral range is $2 \AA$. It is noteworthy that bandpass of this etalon is still about $1+$ times the laser cavity mode spacing. One might wonder how it produces single mode operation. The reason is that light passes through the etalon many times before it escapes the laser cavity. After each pass the light which was transmitted by the etaion (and the prism) is amplified by the active dye medium. Excited dye molecules will see the largest photon flux in modes with the least loss and will be stimulated to radiate into these modes before they can be stimulated to radiate into modes with smalier photon flux. This process is known as mode competition. 39 For maximum power, the desired mode should lie at the center of the etalon pass band, which should in turn lie at tiie center of the prism passband. In the Spectra Physics 370581 laser both etaion and cavity lengths can be changed piezoelectricaliy in such a way that the lasing frequency can be continuous1y tuned over several hundreths of an Angstrom.

The principal limitation on laser linewidth is the precision with which the cavity length can be maintained. Such things as acoustic noise from 
the circulator pump transmitted by the liquid, sound transmitted through the air, vibration from the water cooling within the pump laser and other sources contribute to its actual linewidth by causing the laser cavity length to oscillate on very short time scales. For the present work, it was sufficient to reduce the laser linewidth from $40 \mathrm{MHz}$ to $6 \mathrm{MHz}$ : the chief source of linewidth broadening was acoustic coupling of the laser cavity to the dye circulator pump. This was reduced by introducing into the high pressure side of the dye line the viscoelastic damper shown in Figure 9. Viton 0-rings were used as damping medium because of their superior viscous properties at the $200-400 \mathrm{~Hz}$ acoustic frequency of the pump. ${ }^{40}$ Their performance was experimentaily verified to be far superior to Buna $\mathrm{N}$, whose damping characteristics fall oif badiy above about $20 \mathrm{~Hz} .{ }^{40}$ The dye laser cavity was decoupled from vibration generated by the water cooling within the pump laser by removing the mounting platform which came with the laser for ease of aligning the pump laser with the dye laser. Instead, both lasers were mounted magnetically to a very heavy iron table.

The problem of long term absolute frequency stability needed for isotope separation is somewhat less subtle. Two things cause the laser frequency to drift; temperature change and pressure change. Change in barometric pressure will cause the frequency to drift at a rate of $\sim 188$ $\mathrm{MHz}$ /torr due to the change in the index of refraction of air with pressure. Temperature change likewise causes index change but more significantly, it causes consituent materials of the laser to expand and contract, thereby altering physical dimensions of the cavity. For the purposes of these experiments, it was sufficient to maintain room temperature to $\pm 1^{\circ} \mathrm{F}$ and to insulate both lasers against air currents. With these precautions the lasers can operate for tens of minutes with less than $5 \mathrm{MHz}$ drift. If 


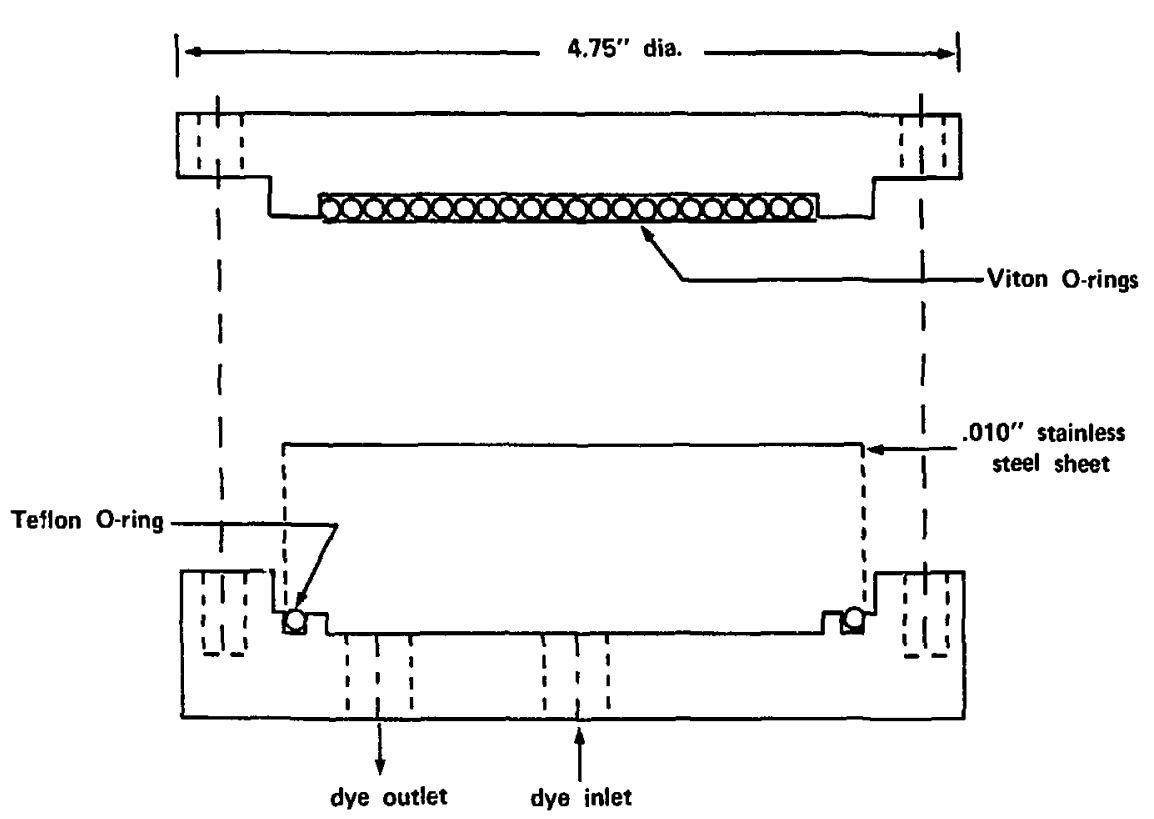

FIGURE 9: Viscoelastic noise damper to prevent acoustic coupling between dye circulator and laser cavity. 
such statility were required for much longer periods, however, the lasers might have to be hermetically sealed inside a thermally controlled container.

Whil such precautions were not necessary for the lasers, the optical spectrum analyzers which were used to monitor laser frequency and which function :d as frequency standards in the spectruscopic measurements reported below were all sealed and temperature controlled. Analyzers used in experiments were a Spectra Physics 470 with $2 \mathrm{GHz}$ free spectra range and a Tropel 240 with $1.5 \mathrm{GHz}$ free spectral range. Mirror spacing is controlled piezoelectrically in both analyzers. Each was mounted inside, but insuiated from, a brass tube whose temperature was precisely contirolled. The arrangement is shown in Figure 10. A thermistor was cemented to the inside of the tube. Its resistance was monitored by a Yellow S,srings Instrument model 72 proportional temperature con:oller which regulated the current to nichrome resistænce wire wourd around (but electrically insulated from) the brass tube. Since the brass tube was several inches longer than the analyzer, this construction allowed even and regular heating of the analyzer through the inter vening annular region of air. The brass tube with the analyzer inside was then the. ually insulated using polyurethane foam and a "bakelite" container wi in end pieces sealed $w$ ith rubber gaskets. A lens in the front end piece permitted entry of light. Electrical feed throughs were sealed with "silastic" silicone rubber adhesive. These precautions gave absolute frequency stability of better than $\pm 2 \mathrm{MHz}$, monitored at 5 to 10 minute intervals over periods of several hours. Stability measurement over longer periods was not attempted but there is no reason to believe such stability cannot be indefinitely maintained. 


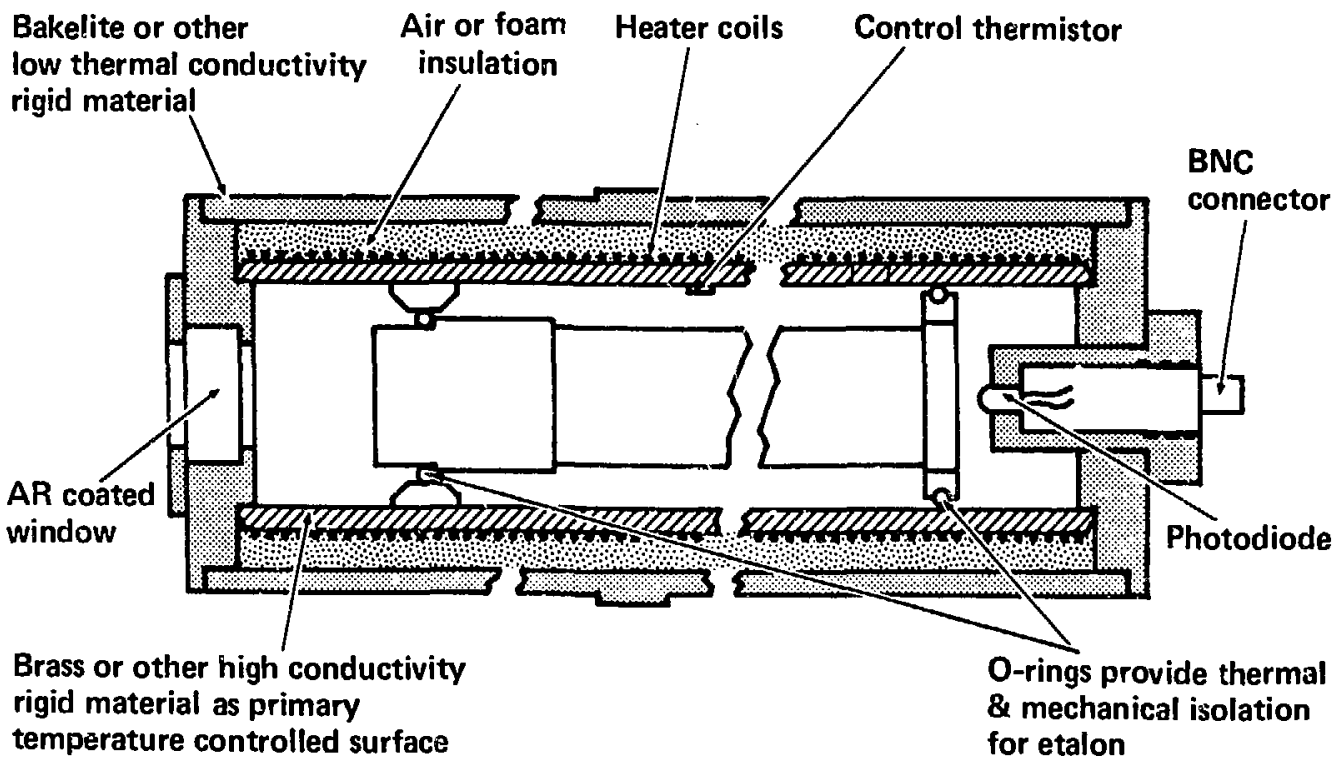

FIGURE 10: Temperature controlled housing for optical spectrum analyzers. 
The laser used to generate $5826 \AA$ radiation deserves brief description. Its optical design is shown in Figure $110^{42}$ It is a coherent Radiation Modei 490 dye laser, with a birefringent tuning element in place of a prism. ${ }^{43}$ This element narrows laser oscillation to a $1 / 4 \AA$ wide pass band. The laser was modified to run single line by adding two etalons inside the cavity. The spacing of the etalon with the smaller passband could be piezoelectrically changed. The manufacturer's nontunable mirror mount was replaced by a Burleight Model PZ81 piezoelectrically driven mirror mount. With these alterations, $60 \mathrm{~mW}$ of monchromatic output power with 4 watt all line Ar laser input was obtained and continuous tuning by manual voltage adjustment was possible. The linewidth attained was $2 \times 10^{-5} \AA$ ( $2 \mathrm{MHz}$ ), which is to be compared with the $3 \mathrm{MHz}$ linewidth of che $5826 \AA$ barium transition. This small linewidth was possible because the solverit, ethylene glycol, has high viscosity and does not transmit any significant circulator pump noise.

It is worth noting that the cavity length in the Coherent Radiation laser was much more stable than the Spectra Physics laser. The large mass of the Invar rod (2" diameter) on which optical elements were mounted prevented any significant expansion on the time scale of room temperature fluctuations. The Spectra Physics laser uses quartz structural members. Quartz has superior thermal expansion properties to invar but, either because these are so small (1/2" diameter) or because they do not actually control the cavity length (fluctuations in the latter being determined by the aluminum end plates, for example), the cavity length is much more subject to variation with changes in ambient temperature in the Spectra Physics laser. 


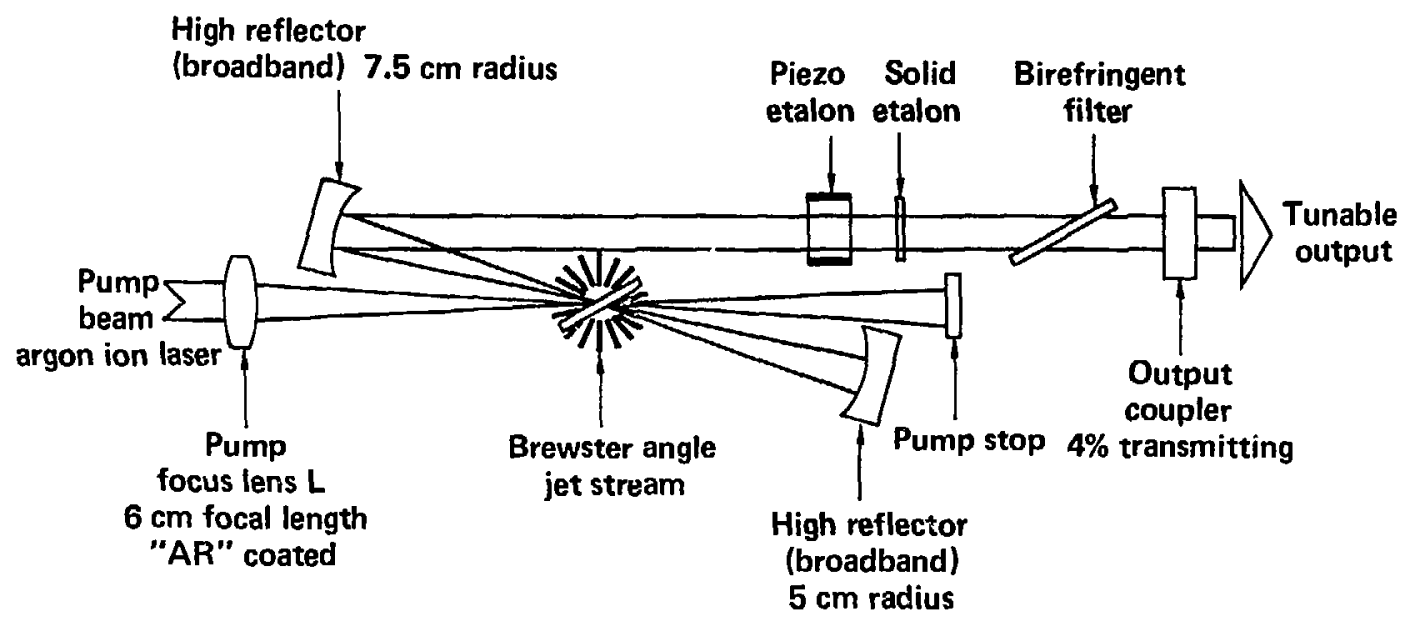

FIGURE 11: Optical design of Coherent Radiation Model 490 dye laser. 
48

In the Coherent Radiation dye lasers, the active region of the dye is not contained in a cell with windows as it is in the Spectra Physics laser. Instead the dye is pumped through a nozzle which projects the dye solution into a free standing jet in the active region. The jet is rectangular in cross section and tilted with respect to the pump beam at Brewster's angle. The lack of an enclosing surface with a resulting layer of quasi-stationary dye means that much more pump energy can be coupled into the dye without burning the dye in the surface layer. For example, with 25 watts of pump power many hundred milliwatts of single mode output can be achieved even with blue or red dyes. 


\section{EXPERIMENTAL APPARATUS}

A diagram of the experimental setup is given in Figure 12. Lasers and spectrum anajyzers have just been described.

The barium is evaporated inside the vacuum system with residual pressure $\approx 10^{-7}$ torr. Vacuum is maintained with a standard LN trap, 6" oil diffusion pump, and forepump combination.

The barium is held inside the double crucible. The crucible and the oven are shown in Figure 13. The inner crucible has an outer diameter of $1 / 2 "$ and is $1 / 2 "$ high. It is made of $1 / 16^{\prime \prime}$ thick molybdenum with a sharp upper edge to prevent liquid barium from migrating over the wall and into the outer crucible (and out of the outer crucible in the same way). The outer crucible has an outer diameter of $3 / 4 "$. It is $3 / 4 "$ high and $1 / 8^{\prime \prime}$ thick. A molybdenum top was machined to fit tightly over the outer crucible. The top had a $0.05 \mathrm{~cm}$ diameter hole in it from which barium vapor could effuse.

The crucibles are radiatively heated from a surrounding resistively heated molybdenum jacket. Temperature is monitored using a Pt-Rh thermocouple located inside the pedestal which supports the crucibles. The heating element is surrounded by give 10 mil thick tungsten foil radiation shields to prevent excessive heat loss.

Twelve centimeters above the crucible there is a slot $0.05 \mathrm{~cm}$ wide and $0.2 \mathrm{~cm}$ long, defined by two razor blades, which collimates the effusive atomic vapor. The divergence of the resulting atomic beam is $\sim 8 \mathrm{mrad}$. With a vapor density of $2 \times 10^{16}$ atoms/cc inside the crucible, approximately $3 \times 10^{13}$ atoms $/ \mathrm{sec}(0.7 \mathrm{mg} /$ day) pass through the collimation slot. No effort was made to maximize throughput, al though only about $1 \%$ of the dye laser power was scattered in the process of separating the abundant ${ }^{138} \mathrm{Ba}$ 


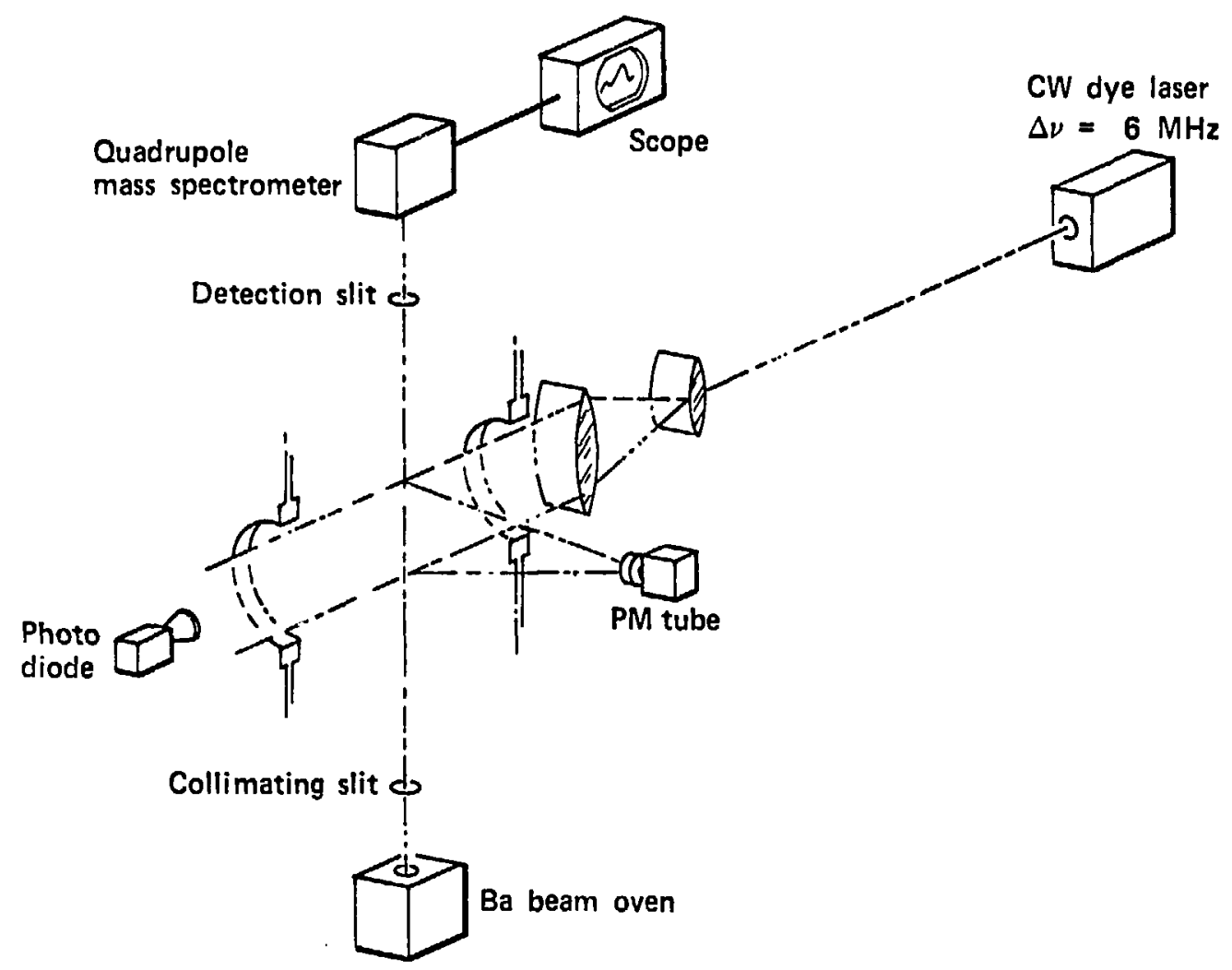

FIGURE 12: Experimental Isotope Separation Set Up 


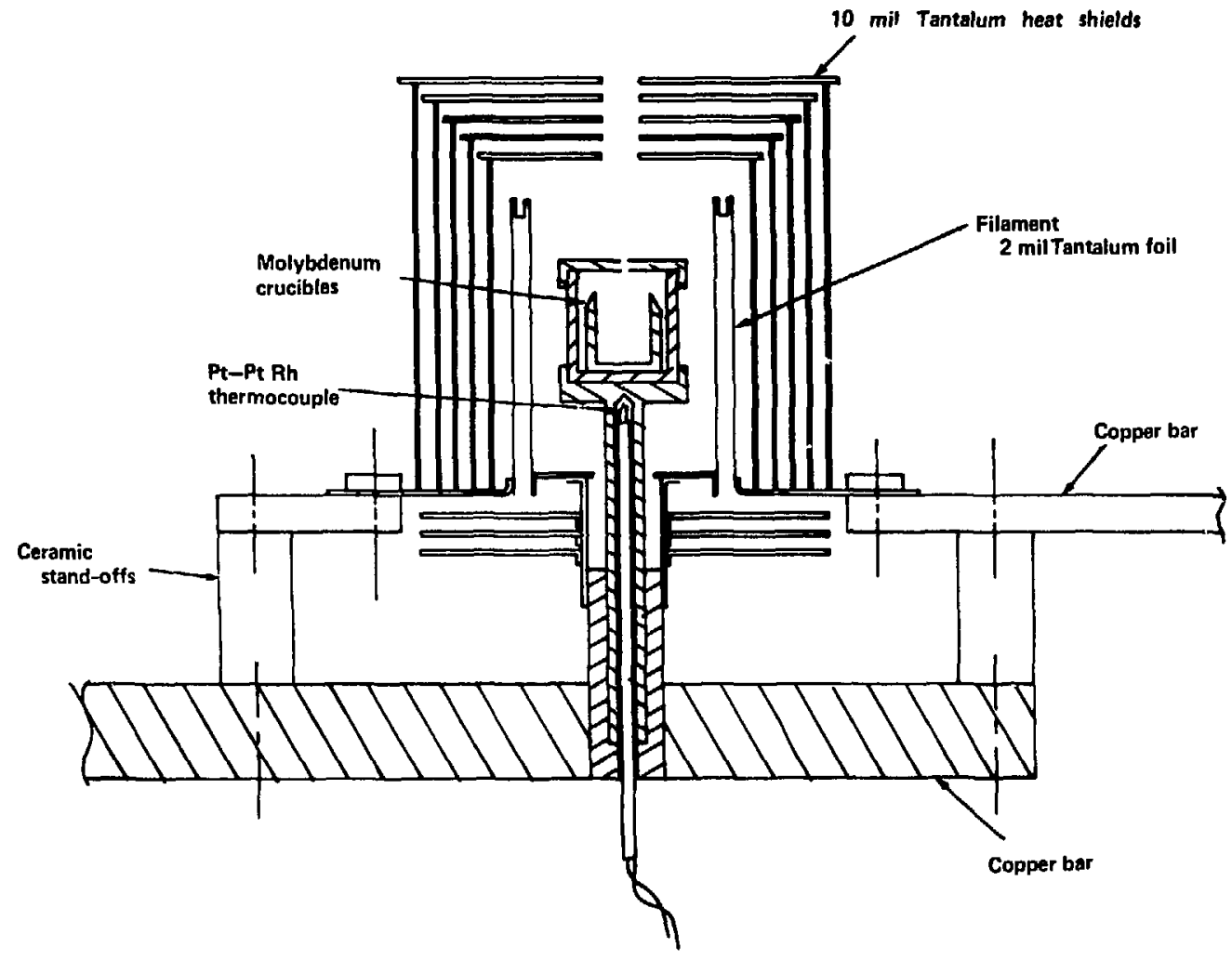

FIGURE 13: Barium Vapor Source 
isotope (and much less for other isotopes). Scaling of the barium system is, of course, rather simple in principle. For example, $5 \mathrm{~cm}$ wide effusion slats (instead of holes) could be stacked 100 deep. Then 100 dye laser beams side by side could be used to separate roughly $3 \times 10^{17}$ atoms per second (or $7 \mathrm{gm} / \mathrm{day}$ ). The scaling problem was considered to be quite secondary to the problem demonstrating the method and defining its limitations. It should be noted, however, that the photodeflection method employs bound state transitions which have very large ábsorption cross sections and, as such, does not require high power lasers. In particular, comparatively low power lasers can be employed in photodeflection schemes, while other methods which involve ionization or dissociation require lasers more than $10^{7}$ times more powerful. They, therefore, must use pulsed lasers instead of continuous lasers. Scaling and efficiency considerations then involve problems of average laser power as well as simple unit replication.

Just above the collimater the green laser beam intersects the atomic beam at a right angle (alignment procedures permitted definition of a right anble to better than one part in fifty). The green laser beam, initially $3 \mathrm{~mm}$ in diameter, is expanded through a cylindrical telescope by a factor of 10 . The reason for expanding the beam is that the $5636 \AA$ barium resonance is saturated at an intensity of about $100 \mathrm{~mW} / \mathrm{cm}^{2}$. At this intensity the absorption rate is approximately equal to the spontaneous emission rate. In the same manner as that discussed on page 18 higher intensities increase the absorption and stimulated emission rates but not the total momentum transfer rate which is limited by the spontaneous emission rate. Furthermore, the excess photon flux is not scattered whereas in an expanded beam, whose intensity is below the saturation intensity, those same photons can be scattered by the atomic beam and produce a corresponding deflection. 
The green laser beam entered the vacuum chamber through a 3" diameter 3/4" thick AR coated window and exited via a similar window. In the experiments which employed the orange laser, this beam used the same windows. It also intersected the atomic beam at a right angle. Radiation scattered by the atomic beam was monitored by two photomultiplier (PM) tubes (RCA 931A), mounted on opposite sides of the atomic beam. Light entered each photomultiplier via a long straight tube which excluded stray radiation. A cutoff filter to exclude $5536 \AA$ light was placed in front of one tube which then became 40 times more sensitive to $5826 \AA$ radiation than to $5536 \AA$ radiation. No filter was necessary for the other detector, as the scattered green radiation was always much more intense than the scattered orange radiation.

Twenty centimeters above the collimating aperture, a second aperture was placed in the path of the atonic beam. Its purpose was to pass either the separated or the unseparated portion of the atomic beam, while blocking the other portion. The aperture was defined by two razor blades $0.4 \mathrm{~cm}$ apart. They were mounted on a platform which could be translated back and forth on the deflection (laser) axis from outside the vacuum chamber by use of a rotary motion feed-through driving a worm gear.

Directly above the second aperture was the ionizer of the EAI Model 200 quadrupole mass spectrometer. The axis of the quadrupole was perpendicular to the atomic beam. The spectrometer head was mounted in such a manner that it could be moved slightly, permitting its signal to be maximized by optimizing the position of the atomic beam within the ionizer. 


\section{EXPERIMENTAL RESULTS}

A. Absorption Spectrum of Natural Barium on the $6 s^{2} 1_{S_{0}}-6 s 6 p P_{P}$

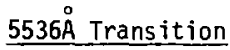

To observe the absorption spectrum of natural barium on the $5536 \AA$ transition, the Spectra Physics dye laser frequency was swept linearly through the atomic resonance using the piezoelectrically driven cavity mirror mount and etalon spacer. The intensity of radiation scattered from the atomic beam was monitored by the photomultiplier tube and displayed on an oscilloscope. The resulting absorption curve is given in Figure 14 . Six peaks are observed. The largest peak must be assigned to the abundant (72\%) ${ }^{138} \mathrm{Ba}$ isotope, and the positions of the other peaks are referred to the center of this peak. Other absorption peaks are observed at about 60 , 130, 280, 320 and $530 \mathrm{MHz}$. Since there are a total of nine components in the spectrum of the abundant barium isotopes, certain observed peaks must contain more than one component. Isotopic determination can be made using enriched samples, which are very expensive since such samples are available only from electromagnetic separation processes (i.e. Calutron). However, the experimental arrangement described in the preceding section (Figure 12) permitted mass analysis of the separated atoms and hence assignment of isotopic composition to each absorption peak (see Section V.C. and V.D.). 


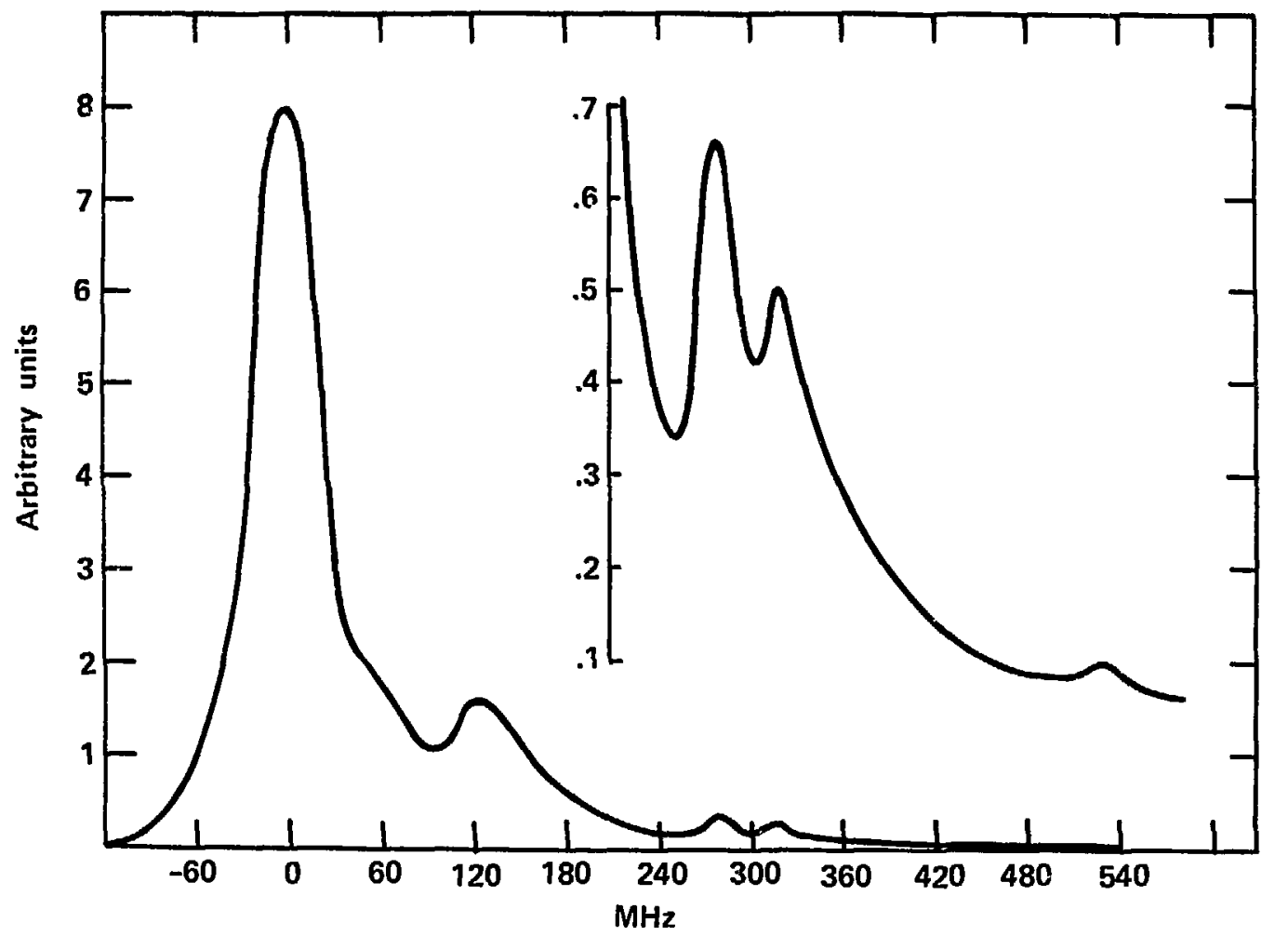

ज

FIGURE 14: Absorption Spectrum of Naturat Barium 


\section{B. Observation of Metastable State Accumulation}

The intensity of the laser beam after passing through the cylindrical telescope was about $50 \mathrm{~mW} / \mathrm{cm}^{2}$. It illuminated the atomic beam along a $3 \mathrm{~cm}$ path (see Figure 12). When the laser frequency was swept through the ${ }^{138} \mathrm{Ba}$ resonance, the following process occurred. As the laser frequency approached the ${ }^{138} \mathrm{Ba}$ resonance 1 ine center, the scattered light from all parts of the illuminated atomic beam increased in intensity. When laser frequency got within about $10 \mathrm{MHz}$ of exact resonance, however, the upper part of the illuminated atomic beam began to dim while the lower part continued to become brighter. At exact resonance only the lower, $1 / 3$ of the 111 uminated atomic beam continued to scatter brightly while the rest fluoresced only quite dimly. As the laser frequency continued past the ${ }^{138} \mathrm{Ba}$ resonance the process just described happened in reverse.

The explanation for the dimming of the upper part of the illuminated atomic beam is that most ${ }^{138} \mathrm{Ba}$ atoms have fallen into the $6 \mathrm{~s} 5 \mathrm{~d}{ }^{7} \mathrm{D}_{2}$ state in the first centimeter of the illumination region and can no longer scatter $5535 \AA$ radiation. This effect is not due to Doppler shift as a result of deflection because, when a second laser operating at $5826 \AA$ was used to depopulate the metastable state (see Section II), the upper portion of the atomic beam no longer diminished in $5536 \AA$ fluorescent intensity.

An experiment was undertaken to quantify the metastable state accumulation process. The original experimental setup was changed as illustrated in Figure 15. The mass spectrometer was removed from its port on the vacuum chamber and the slit just below the ionizer was renoved as well. A window was put on the vacant port. The green laser beam was split before entering the cylindrical telescope so that about $5 \%$ of the original beam intensity could be directed chrough the new window to illuminate the atomic 


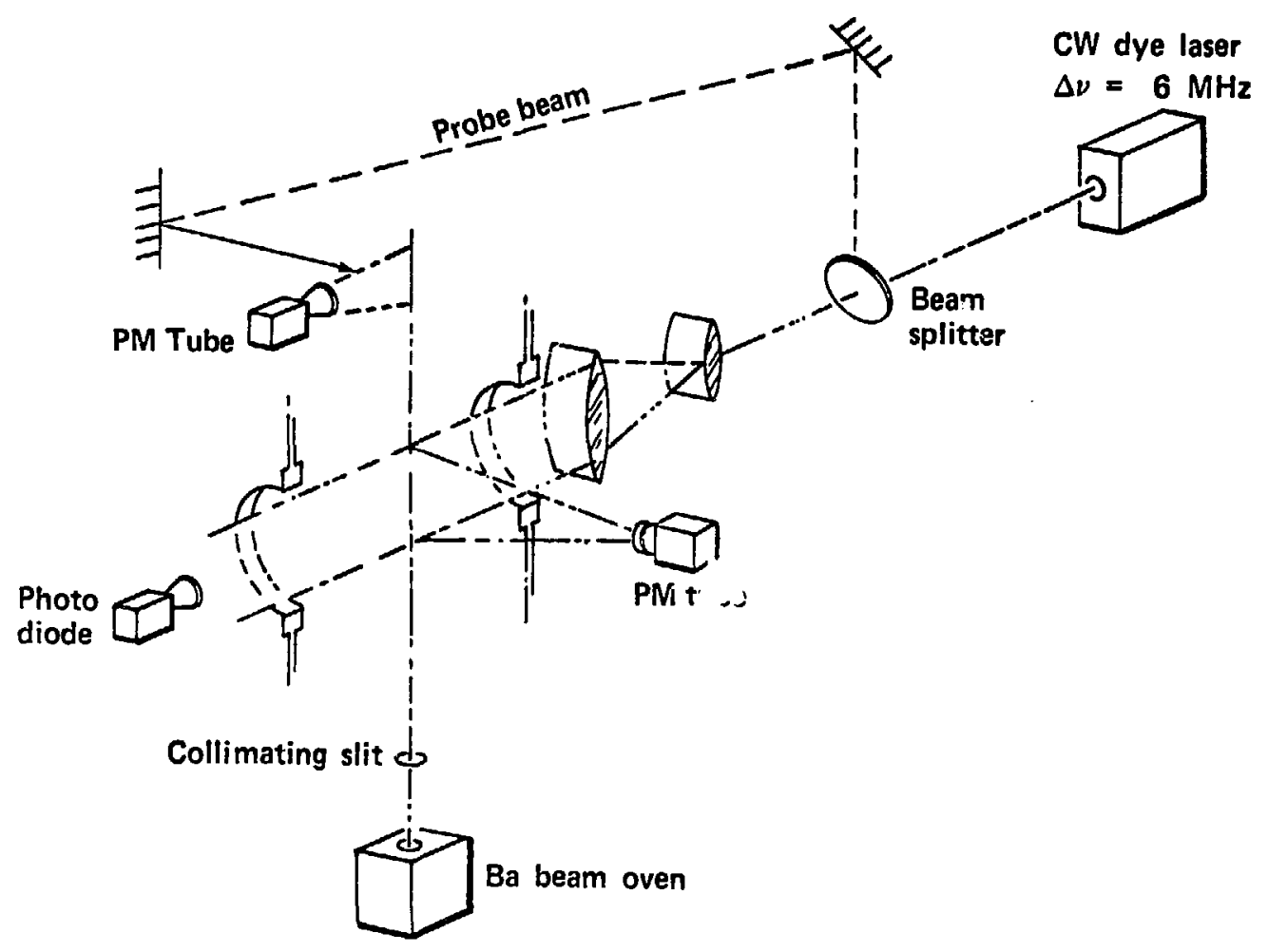

FIGURE 15: Experimental arrangement used to measure accumulation of atums in the 6s5d1D metastable level and the $6 p 5 d^{l} p-$ $5 d^{2}$ oscillator strength. 
beam at a point about $20 \mathrm{~cm}$ above the ccllimatiun slit. The atomic and probe laser beams again intersected at right angles. Another PM tube was aimed at the new intersection to detect $5536 \AA$ fluorescence from this part of the beam.

Figure $16 a$ is a trace of this fluorescence intensity vs. frequency with the main laser blocked off at the telescope. Figure 16a is, of course, identical to Figure 14. Figure $16 \mathrm{~b}$ is again a trace of scattered intensity vs. frequency but the main laser beam is no longer blocked and the metastable accumulation process occurs in the lower interaction region. This causes a dip in scattered probe laser intensity exactly at the ${ }^{138} \mathrm{Ba}$ absorption center. The amplitude at the dip is $2.5 \mathrm{mV}$ while the amplitude of the peak in Figure $16 \mathrm{a}$ is $85 \mathrm{mV}$. Thus only about $3 \%$ of the ${ }^{138} \mathrm{Ba}$ enters the probe laser beam in the ground state. The rest must be in the $6 s 5 d{ }^{1} D_{2}$ metastable state. It is apparent, therefore, that metastable state accumulation will limit the efficiency and extent of isotope separation without some means of netastable state depopulation. 


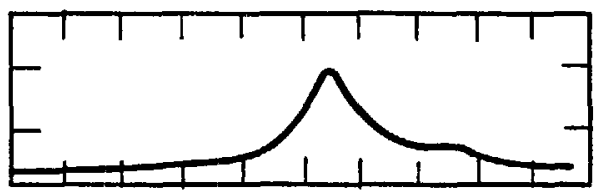

a

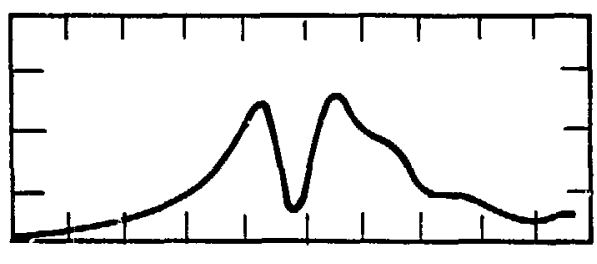

b

\section{$50 \mathrm{mV} / \mathrm{cm}$}

\section{$10 \mathrm{mV} / \mathrm{cm}$}

FIGURE 16: Quantitative measureiriant of metastable state accumulation a) probe laser signal vs. frequency with main laser off

b) probe laser signal vs, frequency with, main laser on. 
C. Isotope Separation Without Metastable State Depopulation

Isotope separation without metastable state depopulation has been observed in the experimental arrangement of figure 12. The electron energy in the mass spectrometer ionizer was set at $10 \mathrm{eV}$. Such a low energy had the advantage of eliminating hydrocarbon background. It is clear from the previous section that most deflected atoms will enter the ionizer in the 6s5d metastable state. At $10 \mathrm{eV}$ electron energy, the ionizer was found to be over twice as efficient in generating ions from metastable atoms than from ground state atoms (see Section V.E.). This further increased the signal to noise ratio. The mass spectrometer output was filtered to eliminate r.f. pickup, amplified using a Tektronix $1 A 7$ plug-in, and averaged by means of a Princeton Applied Research TDH9 Waveform Eductor. In this manner, barium atom densities as low as $3 \times 10^{4} / \mathrm{cc}$ could be reliably detected. A quadrupole unit of more recent design could improve this figure.

Figure 17a shows the mass spectrimeter output when only the deflected portion of the atomic beam was allowed to enter the ionizer of the mass analyzer. The laser frequency was $280 \mathrm{MHz}$ greater than the ${ }^{138} \mathrm{Ba}$ resonance. Figure $17 \mathrm{~b}$ shows the output with laser beam blocked. It is seen that the ${ }^{137} \mathrm{Ba}$ signal in Figure $17 \mathrm{a}$ is greatly enhanced over the background signal in Figure 17b. It should be noted that the barium outside the atomic beam defined by crucible hole and collimation slit, comes from atoms which issued the crucible in a direction which prohibited its direct escape through the hole in the oven's heat shielding (see figure 13). These atoms must make several collisions with surfaces or other atoms before escaping. The resulting "background beam" is defined by the hole in the heat shielding and the collimating slit. A more sophisticated oven design could eliminate the background beam. 

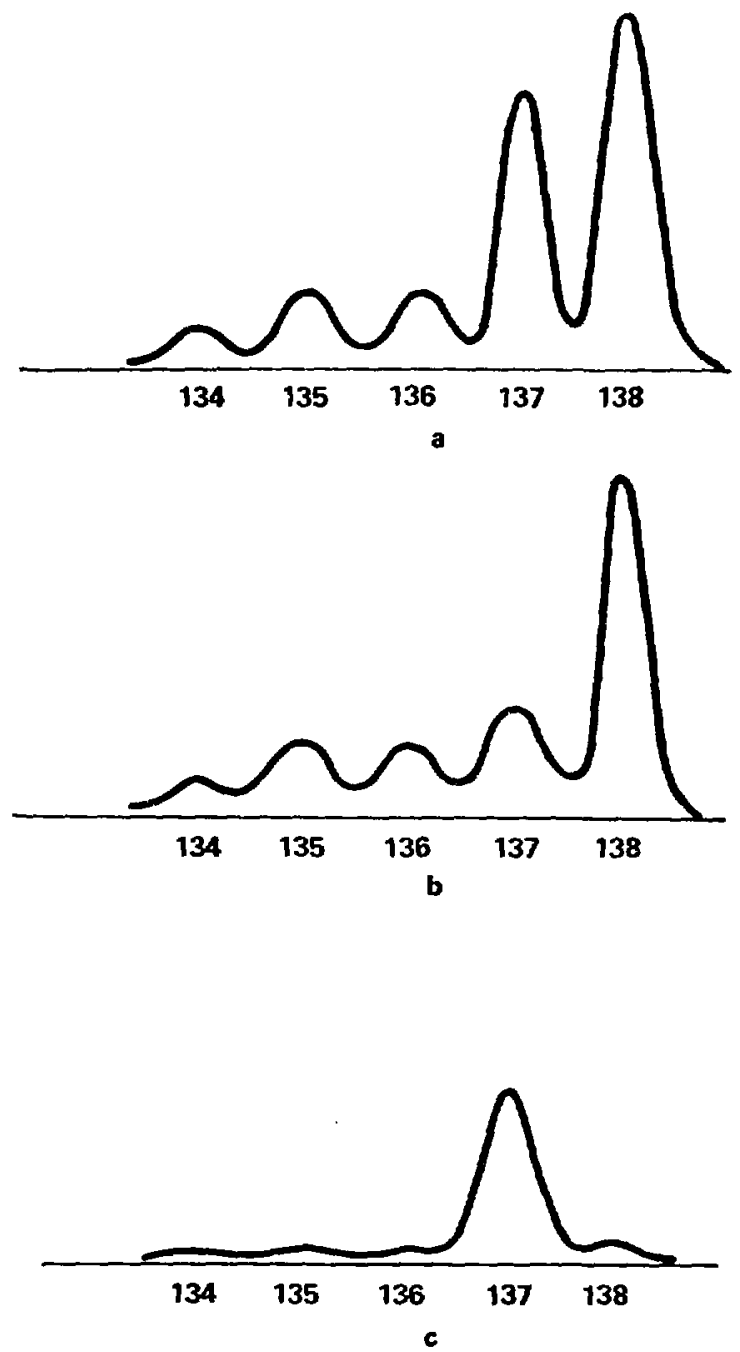

FIGURE 17: Mass anaTyzer signal from atoms downwind of the undeflected atomic beam, b) laser off - only background is detected,

c) signal of a) minus sigral of b). 
If one subtracts amplitudes of Figure 17b from those of $17 \mathrm{a}$ one obtains Figure 17c. Since all peaks in Figure $17 \mathrm{c}$ except for ${ }^{137} \mathrm{Ba}$ are the result of subtracting two large numbers, the relative error is large. It is safe to say, however, that the purity of ${ }^{137} \mathrm{Ba}$ in the deflected beam is greater than $90 \%$ since uncertainty in the height of the peaks is $<5 \%$

Figure $18 \mathrm{a}$ and $18 \mathrm{c}$ shows separation of ${ }^{135} \mathrm{Ba}$ and ${ }^{138} \mathrm{Ba}$. Again, the purity of the deflected species is $290 \%$ in each case. For ${ }^{135_{B a}}$ this represents more than a 13 fold enrichment. Figure $18 \mathrm{~b}$ shows the mass analyzer signal with the laser frequency $130 \mathrm{MHz}$ above the ${ }^{138} \mathrm{Ba}$ resonance. Here several isotopes are separated at once. This is the result of the proximity of three isotopic-hyperfine absorption peaks: ${ }^{135_{\mathrm{Ba}}}$ at $120 \mathrm{MHz}$, ${ }^{136} \mathrm{Ba}$ at $128 \mathrm{MHz}$, and ${ }^{134} \mathrm{Ba}$ at $148 \mathrm{MHz}$ (see next Section). These three absorptions appear as a single peak in the absorption spectrum of figure 14 .

The data of Figures 17 and 18 show that isotope separation has been achieved in the experimental arrangement described. It also shows the potential which the method possesses for producing isotopically pure product. Only when optical overlap occurs is there significant isotopic mixing in the deflected beam. It has been noted several times that the isotopic shifts in barium are anomalously small. Even here, complete isolation of each isotope is possible on at least one line with the exception of ${ }^{136} \mathrm{Ba}$ and ${ }^{134} \mathrm{Ba}$. To obtain a high purity sample of ${ }^{136} \mathrm{Ba}$ it would be necessary to perform the separation in at least 2 steps: (a) separation of ${ }^{136} \mathrm{Ba}$, small impurity of ${ }^{134} \mathrm{Ba}$ and a significant ${ }^{135} \mathrm{Ba}$ impurity at $\Delta v=128 \mathrm{MHz}$, (b) elimination of thee ${ }^{135} \mathrm{Ba}$ impurity by deflection at $\Delta v=322 \mathrm{MHz}$. This now leaves ${ }^{136} \mathrm{Ba}$ with a smal1 ${ }^{134} \mathrm{Ba}$ impurity. Increased purity requires one or more separation steps of $128 \mathrm{MHz}$. $1: 4 \mathrm{Ba}$ purification would require one or more separations at 


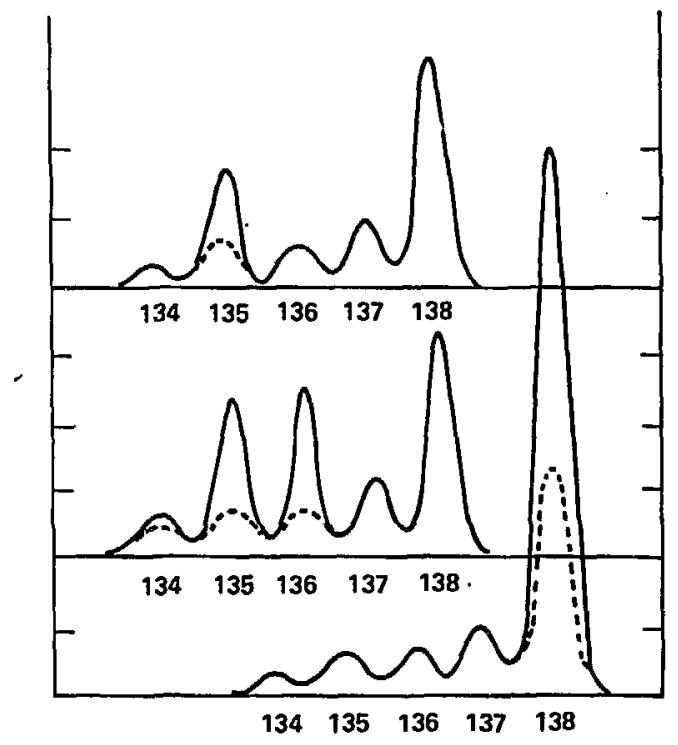

FIGURE 18: Photodeflection of ${ }^{135} \mathrm{Ba}$ at $\Delta v=322 \mathrm{MHz}$; ${ }^{134} \mathrm{Ba},{ }^{135} \mathrm{Ba}$, and ${ }^{136} \mathrm{Ba}$ at $\Delta v=130 \mathrm{MHz}$; 138 Ba at $\Delta v=0$. 
$\Delta v=148 \mathrm{MHz}$. Hence, in spite of anomalously small isotope shifts, separation of each isotope of barium is possible with the laser deflection technique. 
D. High Resolution Spectroscopy in Barium using Photodeflection

In the preceding Section, mass analysis of the deflected atomic beam was used to prove isotope separation. The purpose of the experiment described in the present section is to determine the exact center of the absorption peaks of the various isotopic-hyperfine components of the barium resonance line. This is accomplished using the same setup as was used in the preceding section. Isotopic composition of the deflected atomic beam is now analyzed quantitatively as a function of laser frequency instead of qualitatively at only a few laser frequencies. A plot of the amplitude of each isotope peak on th.: mass spectrometer output as a function of laser frequency gives an absorption curve of each isotopethe same curve as would be found in an atomic beam absorption or emission experiment using enriched samples.

The frequency standard for the experiment was the Spectra Physics Model 470 Optical Spectrum Analyzer described at the end of Section 111. Data was taken by changing the frequency of the laser manually in $8 \mathrm{MHz}$ steps. After averaging for 10 (or, in one run, 20) seconds, a picture of an oscilloscope trace of the output of the TDH9 was taken. Four runs, covering the region between -80 and $+640 \mathrm{MHz}$, were made in this way. The height of isotope peaks of mass 134 to 137 were measured and plotted against optical frequency. Electronic instability of the mass analyzer at low signal levels gave some output amplitude uncertainties. Some of this uncertainty was removed by averaging the four runs so that, except for the weak $F=1 / 2$ component of the ${ }^{135} \mathrm{Ba}$ and ${ }^{137} \mathrm{Ba}$ peaks, the abso7ute error is $\approx 8 \mathrm{MHz}$. The laiter peaks were too close to the sensitivity limit of the mass spectrometer to even be this accurate and the data merely confirms their presence under the peak at $530 \mathrm{MHz}$ in Figure 14. Center 
frequencies for all peaks in the isotopic hyperfine structure of the barium resonance line are given in Table 4 . It is seen that the partially resolved peak in figure 14 nearest the ${ }^{138} \mathrm{Ba}$ resonance is a hyperfine component of ${ }^{137} \mathrm{Ba}$ at $56 \mathrm{MHz}$. This was also the strongest of the three ${ }^{137} \mathrm{Ba}$ peaks as observed on both optical and mass spectrometer data and can therefore be assigned as $F=5 / 2$. The $F=3 / 2$ transition occurs as $280 \mathrm{MHz}$ and the weak $F=1 / 2$ occurs near $530 \mathrm{MHz}{ }^{44}$ The second rather broad peak in Figure 3 is seen to be a combination of three lines which could only be resolved from the mass spectrometer data. This is the first experimental resolution of these peaks without the use of enriched samples. They are ${ }^{135} \mathrm{Ba} \mathrm{F}=5 / 2$ at $120 \mathrm{MHz},{ }^{136} \mathrm{Ba}$ at $128 \mathrm{MHz}$ and ${ }^{134} \mathrm{Ba}$ at $148 \mathrm{MHz}$. The remining components, ${ }^{135} \mathrm{Ba} \mathrm{F}=3 / 2$ and ${ }^{135} \mathrm{Ba} \mathrm{F}=$ $1 / 2$ occur at 322 and $530 \mathrm{MHz}$, respectively. ${ }^{44}$

The accuracy of the experiment was limited by the stability of the mass analyzer electronics. More modern instruments have much improved stability. With a larger number of runs or longer averaging times, the absolute error in frequency assignment can be reduced still more. Ultimately, the accuracy is limited by the linearity of the piezoelectric crystals $(\approx 1 \%)$. 
TABLE 4

Mass Number

138

137

137

137

136

135

135

135

134

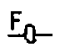

0

$\frac{3}{2}$

$\frac{3}{2}$

$\frac{3}{2}$

0

$\frac{3}{2}$

$\frac{3}{2}$

$\frac{3}{2}$

0
$\underline{F}_{1-}$

1

$\frac{5}{2}$

$\frac{3}{2}$

$\frac{1}{2}$

1

$\frac{5}{2}$

$\frac{3}{2}$

$\frac{1}{2}$

1
148

Shift (MHz)

0

56

281

530

128

120

322

530 
E. Separation Efficiency Without Metastable State Depopulation

It has been seen in Section V.C. that isotopically pure samples of barium can be obtained using photodeflection isotope separation. It remains to determine with what efficiency isotopes can be separated by this method, i.e. What fraction of the desired isotope present in the original beam, can be removed from it.

The experimental arrangement was the same as that of the previous section except that now the slit below the ionizer was adjusted to pass only atoms within the bounds of the original beam. Any atom which was deflected through an angle large enough to emerge from the original beam could not pass through the aperture.

Figure 19a shows the mass analyzer output with the laser blocked. Figure 19b shows the same output with the laser on at the absorption frequency of ${ }^{138} \mathrm{Ba}$. The ${ }^{138} \mathrm{Ba}$ signal with the laser on is only $60 \%$ of what it is with the laser blocked indicating, it would seem, that $40 \%$ of the ${ }^{138} \mathrm{Ba}$ in the atomic beam is being separated when the laser is on. However, since nearly all the atoms which enter the ionizer are in the metastable state, the signal of Figure 19b is spuriously high.

It has been mentioned previously that the ionizer is at least twice as efficient in this case. This result is obtained by comparison of Figure 19b with Figure 19c. In the latter, the second dye laser, operating on the ${ }^{138} \mathrm{Ba} 6 s 5 d \mathrm{D}_{2}-6 \mathrm{p} 5 \mathrm{~d} \mathrm{l}_{\mathrm{P}_{1}}$ transition at $5826 \AA$, was used to depopulate the metastable atoms after they passed through the green beam. The green and orange beams were not allowed to overlap, thus avoiding the possibility that an atom, having been returned to the ground state via the 6p5d level, would then be further deflected by absorption and emission on the $5536 \AA$ transition. 

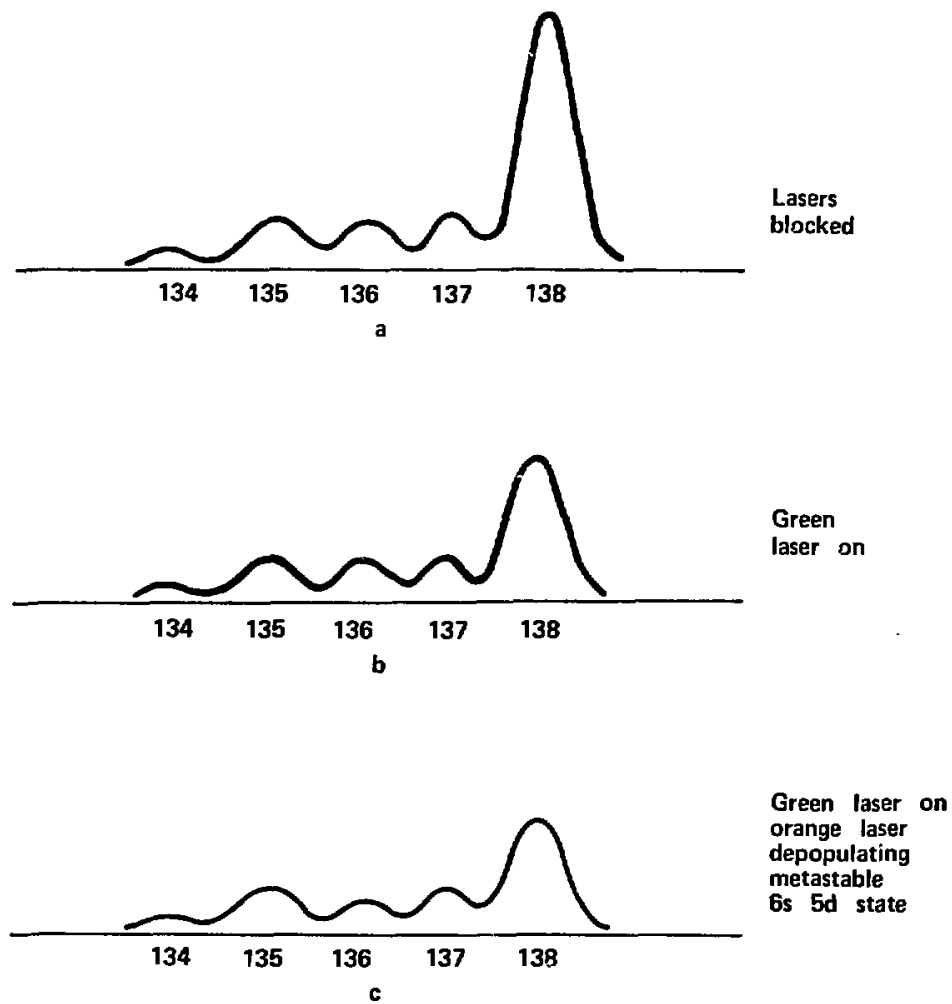

FIGURE 19: Mass analyzer signal resulting from unseparated atoms, a) Signal without deflection,

b) Signal witr deflection is high due to increased ionizer efficiency with metastatie 6s5d atoms,

c) Signal with deflection and orange laser depopulating $6 s 5 d$ state. 
A single pass of the orange laser was sufficient to completely depopulate the metastable state. This is demonstrated by the fact that only in the lower $1 / 3$ or less of the region in which the orange laser beam and the atomic beam intersect, does the $5826 \AA$ radiation scatter strongly. The upper $2 / 3$ or more was very dim indicating absence of $6 \mathrm{~s} 5 \mathrm{~d}$ atoms. In addition, when the $5826 \AA$ beam was reflected back onto the atomic beam, just above its position on the initial pass, no change in the mass spectrometer output was observed.

Depopulation of the metastable state dropped the mass analyzer signal of Figure $19 \mathrm{~b}$ by a factor of 0.7 . This would seem to indicate an ionizer efficiency which is a 1 ittle over $40 \%$ greater for $6 s 5 d$ atoms than for ground state atoms, but decay from the $6 \mathrm{p} 5 \mathrm{~d}$ state into the motastable $5 d^{2}$ state has not yet been accounted for. Clearly, if a significant fraction of $6 s 5 d$ metastable atoms end up in the $5 d^{2}$ metastable state, then the amplitude of Figure $19 \mathrm{c}$ is still spuriously high. An experimental determination of this fraction is presented in Section V.G. It was found that the rate of decay into the $5 d^{2}$ metastable state is $2 / 3$ the rate of decay to the ground state. Thus, $60 \%$ of the metastable atoms will end up in the ground state while $40 \%$ will end up in the $5 d^{2}$ metastable state. If it is assumed that the ionizer efficiency for $6 \mathrm{~s} 5 \mathrm{~d}$ atoms is the same as for $5 d^{2}$ atoms, then the peak amplitude with complete depopulation will be $1 / 2$ that of Figure 19.

Let $A_{6 s 5 d} C=1$ be the signal observed with $C$ atoms/cc in the $6 s 5 d$ state. Similarly, let $A_{\sigma_{s}}{ } C$ be the signal with $C$ atoms/cc in the $6 s^{2}$ state. Knowing the decay probabilities from the 6p5d state and the observed signal we can write 


$$
\begin{array}{r}
0.6 A_{6 s}{ }^{2} C+0.4 A_{5 d} C=0.7 \\
0.6{ }_{6 s}{ }^{2} C+0.4=0.7 \\
A_{6 s}{ }^{2} C=0.3 / 0.6=0.5
\end{array}
$$

The $5 d$ electrons in the $6 s 5 d$ and $5 d^{2}$ configurations are essentially identical since the $5 d^{2}$ level lies almost exactly twice as far above the ground state in energy as the 6s5d level (23062 $\mathrm{cm}^{-1}$ vs $11395 \mathrm{~cm}^{-1}$ ). Certainly the presence of the second $5 d$ electron in the $5 d^{2}$ metastable state will increase the ionization rate and may result in greater ionizer efficiency if the latter is not already close to unity. To the extent that it does, the $70 \%$ number is a lower limi on the separation efficiency. The corrected ${ }^{138} \mathrm{Ba}$ signal with the $c$ een laser on is then only $30 \%$ of the ${ }^{138} \mathrm{Ba}$ signal with the laser off. This indicates that at least $70 \%$ of the ${ }^{138} \mathrm{Ba}$ is actually being separated without metastable state depopulation. 
F. Branching Ratio into the $6 s 5 d{ }^{1} D_{2}$ Metast:ble State - A Theoretical Treatment of Separation Efficiency

The separation efficiency can be calculated from first principles. The theoretical separation is a function of the branching ratio into the 6s5d ${ }^{1} D$ level-rate at which atoms decay from the $6 s 6 p{ }^{1} P_{1}$ level to the metastable $6 s 5 d d^{\prime} D_{2}$ level divided by the rate at which they return io the ground state. Since the separation efficiency and all parameters except the branching ratio are known from experiment, the value of the branching ratio can be determined.

The derivation which follows assumes that a $11{ }^{138} \mathrm{Ba}$ atoms end up in the $6 \mathrm{~s} 5 \mathrm{~d}$ metastable state. The probability that an atom absorbs and reradiates spontaneously to the ground state $n$ times before falling into the metastable state after the $(n+1)^{\text {st }}$ absorption is

$$
\begin{aligned}
& \frac{1}{r+1}\left(\frac{r}{r+!}{ }^{n} \text { where } r=\frac{\text { spontaneous decay rate to ground }}{\text { spontaneous decay rate to metastable }}\right. \\
& \frac{1}{r+1}\left(\frac{r}{r+1}\right)^{n}=\frac{1}{r+1} e^{-n x} \text { where } x=\text { en } \frac{r+1}{r} \backsim \frac{1}{r} \text { for large } r \text {. }
\end{aligned}
$$

Let an atom have velocity $v$ and inclination e with respect to the atomic beam axis. Let the atomic beam have divergence $\theta_{0} \ll 1$. It will be separated if

$$
n \geq \frac{c}{h v} m v\left(\sin \theta-\cos \theta \tan \frac{\theta_{0}}{2}\right) \sim \frac{c}{h_{v}} m v\left(\theta-\frac{\theta_{0}}{2}\right)
$$

Let $\zeta=\theta-\frac{\theta_{0}}{2}$. The probibility that an atom with velocity $v$ at an angle $\zeta$ is separated from the original atomic beam is 


$$
P(v, \zeta)=\frac{1}{r+1} \int_{n(v, \zeta)}^{\infty} e^{-n x} d n \simeq e^{-n(v, \zeta)}
$$

where $n(v, r)$ must be large to justify integration instead of summation. The probability that an atom has velocity between $v$ and $v+d v$ with angle between $\zeta$ and $\zeta+d \zeta$ is

$$
\frac{v^{3} e^{-m v^{2} / 2 k T} d v}{\int_{0}^{\infty} v^{3} e^{-m v^{2} / 2 k T} d v} \times \frac{d \zeta}{\theta_{0}}
$$

where $\theta_{0}$ is the full atomic beam divergence. Therefore the fraction of the total atomic distribution separated is

$$
\varepsilon=\frac{\int_{0}^{\theta_{0}} \int_{0}^{\infty} v^{3} e^{-m v^{2} / 2 k T} e^{-n(v, \theta) x} d v d \theta}{\theta_{0}^{\infty} \int_{0}^{\infty} v^{3} e^{-m v^{2} / 2 k T} d v}
$$

The integrations are performed in Appendix A. The result is

$$
\begin{gathered}
\varepsilon=\frac{1}{2}+\frac{\sqrt{\pi}}{2} \frac{\beta}{x}-\frac{\sqrt{\pi}}{2} \frac{\beta}{x} e^{\alpha^{2} x^{2}}+\frac{\sqrt{\pi}}{2} \frac{\beta}{x} e^{\alpha^{2} x^{2}} \text { erf }(\alpha x) \\
-\frac{\sqrt{\pi}}{4} \frac{x}{\beta} e^{\alpha^{2} x^{2}}+\frac{\sqrt{\pi}}{4} e^{\alpha^{2} x^{2}} \text { erf }(\alpha x)
\end{gathered}
$$

where

$$
\begin{aligned}
& B=\frac{l}{(2 m k T)^{1 / 2}} \frac{h_{v}}{\mathrm{Cg}_{0}},\left(\sim 2.2 \times 10^{-3}\right) \\
& a=\left(\frac{m k T}{2}\right)^{1 / 2} \frac{c_{0}}{h_{v}},\left(\sim 2.4 \times 10^{2}\right)
\end{aligned}
$$


The above expression is quite complicated but its asymptotic behavior is easy to check. Let $x \rightarrow 0$. This means that an atom will almost never decay to the metastable state. Then the last two terms go to zero, the second and third terms cancel and the expression becomes

$$
\begin{aligned}
& \varepsilon \simeq \frac{1}{2}+\frac{\sqrt{\pi}}{2} \frac{B}{x} e^{\alpha^{2} x^{2}} \frac{2}{\sqrt{\pi}} \int_{0}^{\alpha x} e^{-t^{2}} d t \\
& \simeq \frac{1}{2}+\frac{1}{(2 m k T)^{1 / 2}} \frac{h v}{m c \theta_{0}} \frac{1}{x} e^{\alpha^{2} x^{2}} e^{-\alpha^{2} x^{2}} \alpha x \\
& \simeq \frac{1}{2}+\frac{1}{(2 m k T)^{1 / 2}} \frac{h v}{c \theta_{0}}\left(\frac{m k T}{2}\right) \\
& \simeq \frac{c \theta_{0}}{h v} \\
& \simeq \frac{1}{2}+\frac{1}{2}=1
\end{aligned}
$$

Thus, nearly all atoms are separated in the 7 imit that the metastable state is inaccessible from the $656 p p_{p_{1}}$ state.

In the opposite limit, $x \rightarrow \infty$, where excited atoms decay with near unit f. Whability to the metastable state, the expression for $\varepsilon$ can be expanded ${ }^{45}$ and it vecomes

$$
\begin{gathered}
\left.\varepsilon=\frac{1}{2}+\frac{\sqrt{\pi}}{2} \frac{1}{(2 m k T)}\right)^{1 / 2} \frac{h_{v}}{c \theta_{0}}-\frac{1}{2}\left[\left(\frac{h_{v}}{c \theta_{0}}\right)^{2} \frac{1}{m k T}+1\right] \\
\varepsilon=\frac{1}{2}+\frac{\sqrt{\pi}}{2} \frac{1}{(2 m k T)^{1 / 2}} \frac{h_{v}}{c \theta_{0} x}-\frac{1}{2}\left\{\left(\frac{h_{v}}{c \theta_{0} x}\right)^{2} \frac{1}{m k T}+1\right\}\left(1-\frac{1}{2(\alpha x)^{2}}+\frac{3}{4 \alpha^{4} x^{4}}-\cdots\right)
\end{gathered}
$$




$$
\varepsilon_{x \rightarrow \infty}=\frac{1}{2}-\frac{1}{2}=0
$$

Thus the separation efficiency becomes zero for very large branching ratios into the metastable state, as one would expect.

A graph of efficiency as a function of $r \approx \frac{1}{x}$ is presented in Figure 20 . It is seen that an estimated efficiency of 0.7 implies $r \sim 700$ and a branching ratio of about. $\frac{1}{r} \sim \frac{1}{700}$. This is in drastic disagreement with the vilue $r \approx 24$ from the literature. ${ }^{27}$ Since the efficiency of 0.7 is a lower limit, so too is the branching ratio of $\frac{1}{700}$ an upper limit. Consider another, more simple derivation of separation efficiency. Let the atomic beam have a divergence of $\theta_{0}$. An average atom in the beam has a velocity $\bar{v}=\sqrt{\frac{\pi k T}{2 m}}$ and travels at an angle $\frac{\theta_{0}}{2}$ from the downwind edge of the beam. Such an atom will be separated then, if it absorbs more than $n_{0}$ photons where

$$
\begin{aligned}
n_{0} & =\frac{\theta_{0}}{2} m \bar{v} \frac{c}{h_{v}} \\
& =2.7 \times 10^{2} \text { for } \theta_{0}=8 \times 10^{-3}, T=1073^{\circ} \mathrm{K}, \frac{c}{v}=\lambda=5.5 \times 10^{-5}
\end{aligned}
$$

which were the actual experimental parameters. The efficiency of the separation process is just the probability that an atom absorbs $n \geq n_{0}$ photons before falling into the metastable state:

$$
\varepsilon \simeq \frac{1}{25} \int_{n_{0}}^{\infty} e^{-0.04 n} d n=e^{-0.04 n_{0}} \simeq e^{-11} \approx 2 \times 10^{-5}
$$

where $\left(\frac{24}{25}\right)^{n}=e^{-0.04}$ from Section II. 


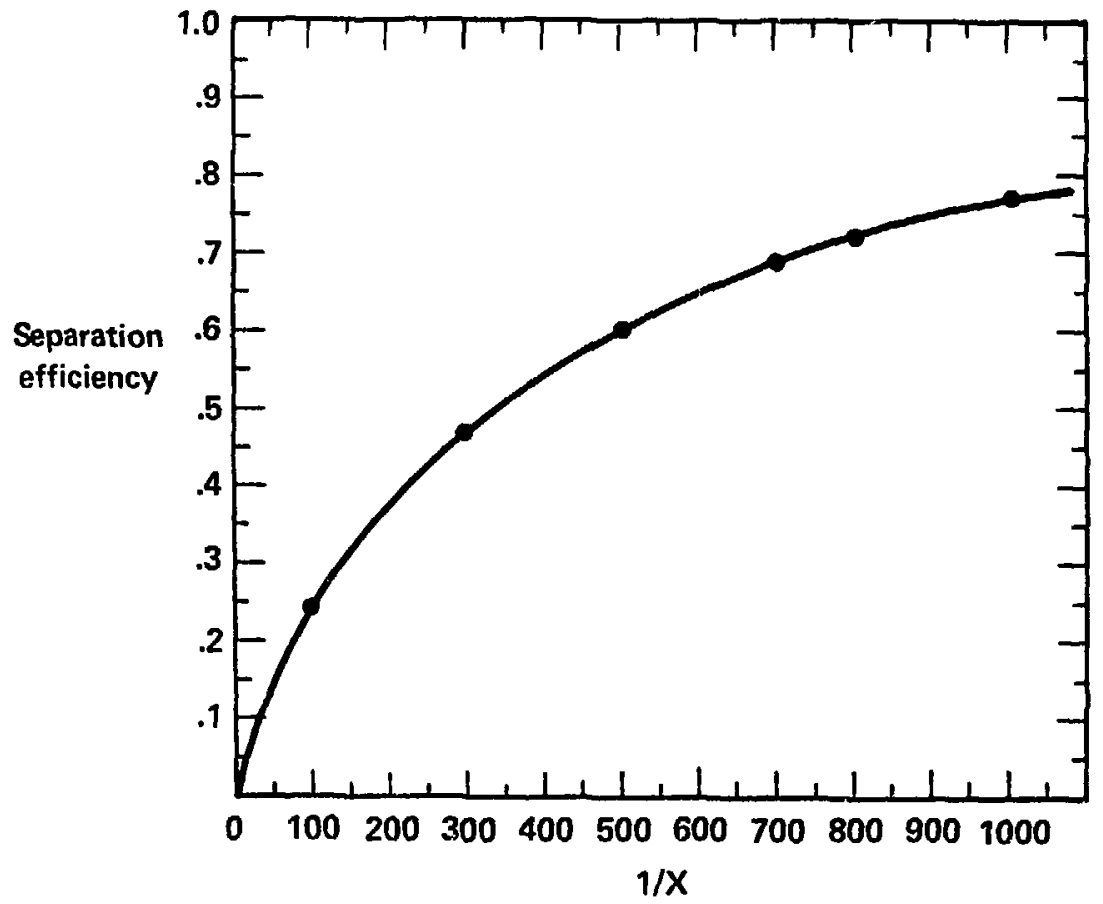

วั

FIGURE 20: Calculation of separation efficiency as a function of $1 / x \sim \frac{\text { decay rate to around state }}{\text { decay rate to metastable states }}$ for experimental parameters. 
This figure of $2 \times 10^{-5}$ for the separation efficiency is again in drastic disagreement with the observed efficiency. Working backwards

$$
\begin{aligned}
e^{-x n_{0}} & =0.7=\varepsilon \\
x n_{0} & =\ln 1.43=0.36 \\
x & =\frac{0.36}{n_{0}}=\frac{0.36}{270}=1.3 \times 10^{-3} \\
\Rightarrow r & \approx 770
\end{aligned}
$$

The answer from this simple analysis is within $10 \%$ of the one derived from the more rigorous analysis above.

Some recent work supports this branching ratio result. In a recent private communication, A. C. Gallagher of the Joint Institute for Laboratory Astrophysics at Boulder reported "at least a factor of 10" discrepancy between the branching ratio implied by his results in connection with a monoenergetic electron source ${ }^{46}$ and the published number, in the same direction as observed here. He pointed out the paper by $P$. McCavert and E. Trefftz which reports a $1 / 600$ branching ratio for this transition on theoretical grounds. 26

The oscillator strength for the $6 s 6 p P_{P_{1}}-6 s 5 d{ }^{1} D_{2}$ transition is found from the branching ratio:

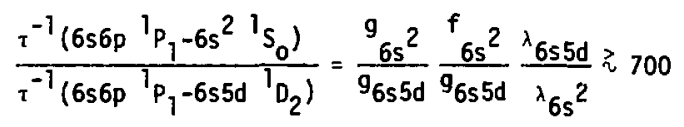




$$
\begin{gathered}
\frac{1}{5} \frac{1.59}{f_{6 s 5 d}}\left(\frac{1.5}{0.55}\right)^{2} \gtrsim 700 \\
f_{655 d} \leq \frac{1.59}{5}\left(\frac{1.5}{0.55}\right)^{2} \frac{1}{700}=3.4 \times 10^{-3}
\end{gathered}
$$

This is to be compared with $\mathrm{f}_{6 \mathrm{~s} 5 \mathrm{~d}} \approx 5 \times 10^{-3}$ reported by McCavert and Trefftz and $f_{6 s 5 d} \sim 0.1$ reported by Miles and Wiese. 27 
G. Branching Ratio and Oscillator Strength into the $5 \mathrm{~d}^{2}{ }^{1} \mathrm{D}_{2}-$ Metastable State

The use of a second laser to depopulate the $655 d^{1} D_{2}$ metastable state in barium is now discussed. For reasons presented in Section II, atoms in the $6 s 5 d d^{1} D_{2}$ state are returned to the ground state via the $6 \mathrm{p} 5 \mathrm{~d}{ }^{1} \mathrm{P}_{1}$ level. Si:ice another metastable state, the $5 d^{2} I_{D_{2}}$, is accessible from the $6 \mathrm{p} 5 \mathrm{~d}^{1} \mathrm{P}_{\eta}$ level, it is necessary to determine how rapidly atoms are transferred into the $5 d^{2}$ metastable state. It is also necessary to establish that the lifetime of this state is indeed large compared to the time during which an atom inu; acts with the lasers: $t \backsim 5 \times 10^{-5} \mathrm{sec}$.

The experiment was conducted in exactly the fashion described in Section VI. B., and Figure 15, ihat is, with the mass analyzer head removed and a green probe laser traversing the chamber in its place. Again, a photomultiplier was used to detect probe laser radiation scattered by the atomic beam. It was noted in Section V.B. that only $3 \%$ of the atoms entering the probe laser beam were in the ground state after irradiation with the green laser in the deflection region. Now, the second laser operating at the ${ }^{138} \mathrm{Ba}$ : $6 \mathrm{~s} 5 \mathrm{~d} \mathrm{C}_{\mathrm{D}_{2}}-6 \mathrm{p} 5 \mathrm{~d} \mathrm{C}_{\mathrm{P}_{1}}$ transition at $5826 \AA^{\circ}$, was used to completely depopulate the 6s5d metastable state. The orange laser intersected the atomic beam just above the deflecting green bean as des.ribed in Figure 21 . This caused the metastable atoms to be distributed be-ween the ground state and the $5 d^{2}$ metastable state. In the event that the brariching ratio into the $5 d^{2} l_{D_{2}}$ state is very small or the latter state has lifetime short compared to the time for an atom to travel the $18 \mathrm{~cm}$ or so from its intersection with the orange laser beam to its intersecsion with thegreen probe beam ( $\left.t \approx 3 \times 10^{-4} \mathrm{sec}\right)$, the full scattered green probe beam intensity would be recovered. 


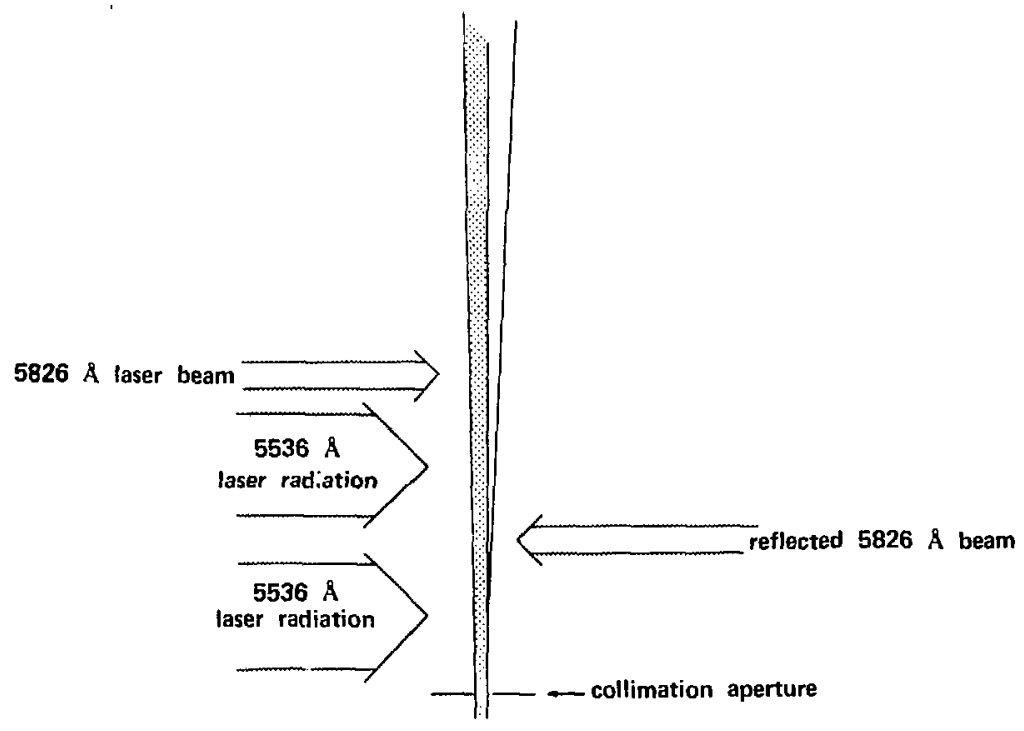

FIGURE 21: Laser beam geometry used to measure $6 \mathrm{p} 5 \mathrm{~d} 1 \mathrm{P}$ - 
The result of a single pass of the orange beam was an increase in scattered intensity to about 0.6 the value with no green in the deflection region. This result implies a lifetime $\tau \gtrsim 3 \times 10^{-4} \mathrm{sec}$ but it cannot yet be asserted either that $\tau \gg 3 \times 10^{-4} \mathrm{sec}$ and or that the signal accurately reflects the branching ratio into the $5 d^{2}$ state.

The experiment can be carried further, however, to determine a branching ratio independent of the lifetime of the $5 d^{2}$ state. A 1/4" section at the center of telescoped green laser beam was blocked with a piece of tape. This permitted the orange beam to be reflected back across the atomic beam between the resulting pair of green beams without any overlap with either of the green beams. This is also illustrated in Figure 21. Atoms which are pumped into the metastable $655 \mathrm{~d}$ state by the lower half of the green beam are distributed between $5 d^{2}$ and ground states by the orange bean which passes between the pair of green beams. Atoms which then enter the upper half of the green beam in the ground state can be pumped back into the $655 d$ metastable state. These now have a second chance to decay to the $5 d^{2}$ state after they are pumped into the 6p5d state by the orange beam which passes above both green beams. Since more atoms end up in the $5 d^{2}$ state with two depopulation steps, the scattered probe laser intensity on the PM tube should be less than that with only one depopulation step. Assume, for example, that all $1{ }^{3} \mathrm{Ba}$ atons end up in the 6s5d state after passing through the lower half of the green beam. The lower orange beam then depopulates the $655 \mathrm{~d}$ level, causing some fraction, $f$, of the atomc to accumulate in the $5 d^{2}$ level with the rest ending up in the ground state. The latter, after passing through the upper half of the green beam, will accumulate in the 6s5d state again, from which $f$ of these will end up in the $5 d^{2}$ state after 
passing through the upper orange beam. The intensity of radiation scattered out of the green probe beam with two depopulation steps should be $1-f-f(1-f)=(1-f)^{2}$ of the full intensity with no deflection (both halves of telescoped green beam blocked). This analysis assumes that no significant fraction of $5 d^{2}$ atoms decay before they pass the probe laser beam. The intensity with only one depopulation step (e.g. with the lower orange beam blocked) should be (1-f) of the full intensity.

In practice, the either half of the telescoped green beam was insufficient to promote all ${ }^{138} \mathrm{Ba}$ atoms to the $655 \mathrm{~d}$ state. It has already been shown that the entire telescoped green beam only drops $97 \%$ of the $138_{\mathrm{Ba}}$ atoms into this state. With a $1 / 4$ " patch of the center blocked, $96.5 \%$ of ${ }^{138} \mathrm{Ba}$ atoms end up in the $6 s 5 \mathrm{~d}$ state while a single portion (either upper or loweri Hil only drop $81 \%$ of the atoms into the $6 s 5 d$ state. These percentages were determined in the same manner as the $97 \%$ figure (see Section V.B.).

Knowing the fraction of ${ }^{138} \mathrm{Ba}$ atoms which enter each orange beam, and having measured the scattered probe beam intensity for one and two depopulation steps, it is possible to determine what the scattered intensity would be with all ${ }^{138} \mathrm{Ba}$ atoms entering each orange beam in the $6 \mathrm{~s} 5 \mathrm{~d}$ level and the intensities should then be related as described above, provided $5 d^{2} \gg 3 \times 10^{-4} \mathrm{sec}$.

Figure 22 shows typical PM outputs for (a) no green in deflection region, (b) both parts of green on and upper orange beam on, and (c) both green and both orange on. This series of pictures was taken five times over. Consistent results were obtained and the average of each, corrected for incomplete metastable state accumulation, is given in Figure 23 with error bars to indicate an approximi a standard deviation in the data. The 


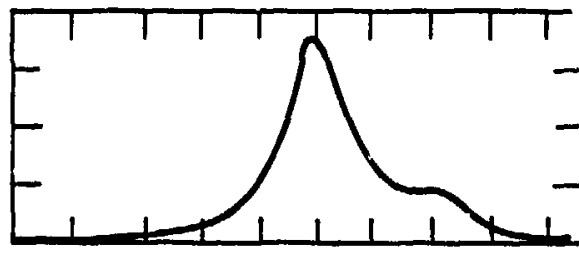

\section{$20 \mathrm{mV} / \mathrm{cm}$}

a

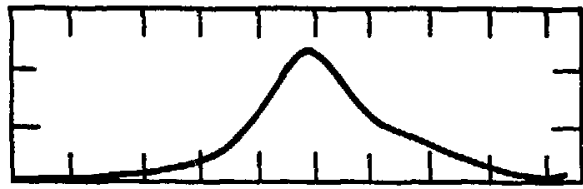

\section{$20 \mathrm{mV} / \mathrm{cm}$}

b

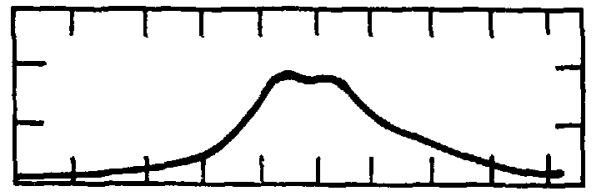

$20 \mathrm{mV} / \mathrm{cm}$

C

FIGURE 22: Intensity of scattered probe laser radiation corresponding to the geometry of Figure 21, a) green laser blocked

b) green laser on and uppermost orange beam on (Figure 21)

c) green laser and both orange beams on. 


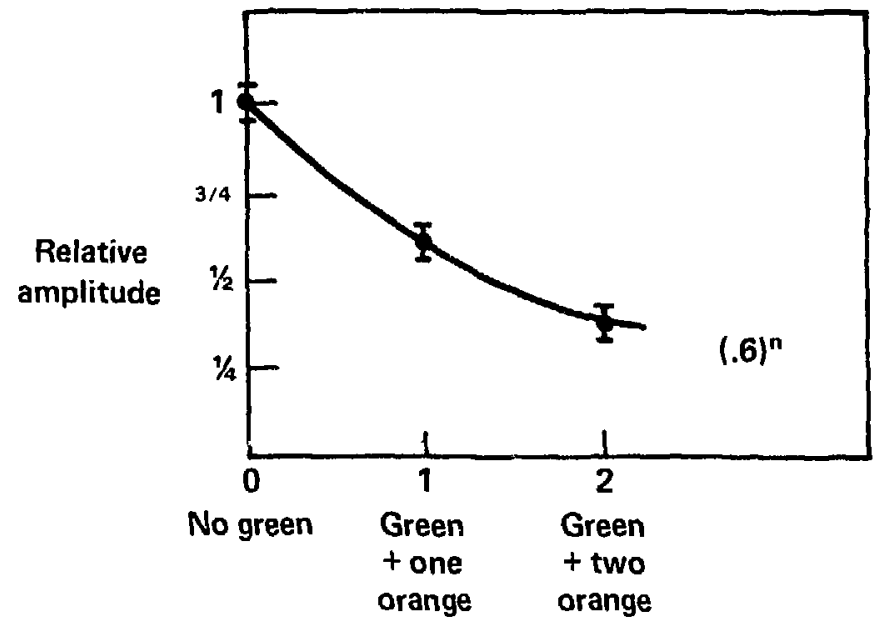

FIGURE 23: Fraction of toms in ground state after zero, one, and two 6s5d depopulation cycles, corrected for incomplete population of $6 s 5 d$ 'D level. 
amplitude of the third point is 0.6 that of the second point which in turn is 0.6 that of the first. This indicates $\mathrm{T} \gg 3 \times 10^{-4} \mathrm{sec}$ (otherwise the two ratios would be different) and gives branching ratio and osciliator strength into the $5 d^{2}$ state from the $6 p 5 d$ :

$\frac{\tau^{-1}(5 d 6 p-6 s 5 d)}{\tau^{-1}\left(5 d 6 p-6 s^{2}\right)}=\frac{g_{6 s 5 d}}{g_{6 s^{2}}} \frac{f_{6 s 5 d}}{f_{6 s^{2}}{ }^{2}} \frac{\lambda_{6 s^{2}}^{2}}{\lambda_{6 s 5 d}^{2}}=5 \frac{0.19}{0.10}\left(\frac{3501}{5826}\right)^{2}=3.43$

using published values for $f_{6 s 5 d}$ and $f_{6 s} 2^{4}$ Let $\tau^{-7}\left(6 p 5 d-6 s^{2}\right)=1$ and $\tau^{-1}\left(6 p 5 d-5 d^{2}\right)=\chi$ which we wish to determine. Then $\tau^{-1}(6 p 5 d-6 s 5 d)=3.43$. The fraction of atoms which end up in the $5 d^{2}$ state on passing through one orange beam is

$$
\frac{x}{4.43+x}+\frac{3.43}{4.43+x}\left(\frac{x}{4.43+x}\right)+\cdots
$$

where the first term is the probability that an atom falls into the $5 d^{2}$ the first time it decays from the $6 \mathrm{p} 5 \mathrm{~d}$, the second term is the probability that it falls back into the 6s5d once before being re-excited and decaying tc the $5 d^{2}$, etc.

$$
\begin{aligned}
& =\frac{x}{4.43+x}\left(1+\frac{3.43}{4.43+x}+\left(\frac{3.43}{4.43+x}\right)^{2}+\cdots\right) \\
& =\frac{x}{4.43+x} \frac{1}{1-\frac{3.43}{4.43+x}}=\frac{x}{1+x}
\end{aligned}
$$

The results of the experiment imply that

$$
1-\frac{x}{1+x}=0.6 \text { so that } x=0.67
$$




$$
\begin{aligned}
\frac{\tau^{-1}\left(6 p 5 d-5 d^{2}\right)}{\tau^{-1}\left(6 p 5 d-6 s^{2}\right)} & =\frac{x}{1}=0.67 \\
& =\frac{g d^{2}}{g} \frac{f d^{2}}{6 s^{2}} \frac{\lambda^{2}}{6 s^{2}} \frac{6 s^{2}}{5 d^{2}} \\
\text { therefore } & { }_{5 d^{2}}=\frac{0.67}{5} \times 0.16 \times\left(\frac{18207}{3507}\right)^{2} \approx 0.58
\end{aligned}
$$

This result for ine oscillator strength depends on the oscillator strength for the $6 p 5 d{ }^{1} p_{1}-6 s^{2}{ }^{1} s_{0}$ transition. In the above calculation the value $f_{6 s^{2}}=0.16$ has been used. This is in agreement with the value of Miles and Wiese 27 very close to the value of 0.14 quoted by Penkin, 48 and intermediate between the values quoted by Dickie and Kelly $y^{29}$ ( $f=0.10$ ) and $\mathrm{Ma}$, Lado and ZuPutlitz ${ }^{29}$ (f $\mathrm{f}_{2}=0.22$ ). The exact value of the oscillator strength of the $6 \mathrm{p} 5 \mathrm{~d}^{6 \oint_{\mathrm{D}_{2}}}$ transition is not needed to understand any of the results quoted here. The essential resuit for present purposes is that the probability that an atom in the $6 p 5 d$ state decays to che $5 d^{2}$ state is $2 / 3$ that for decay to the ground state. 
H. Separation Efficiency with Metastable State Depopulation

An experinent to measure the isotope separation efficiency with metastaule state depopulation was performed. The experiment was identical to that used to measure separation efficiency without metastable siate depopulation (Section V. E.) with the addition of the $5826 \AA$ laser beam, telescoped to cover approximately the same area of the atomic beam. The orange beam was displaced slightly upward with respect to the green beam so that no atoms left the deflection region in the $6 \mathrm{~s} 5 \mathrm{~d}$ state. Each laser was tuned to the ${ }^{138} \mathrm{Ba}$ absorption $2 n$ each of the transitions. The slit in front of the mass analyzer admitted only unseparated atoms to the ionizer and the output of the mass analyzer was recorded with both lasers on and both lasers off. The analyzer output in these two situations is given in Figure $24 \mathrm{~b}$ and $24 \mathrm{a}$ respectively. The ${ }^{138} \mathrm{Ba}$ signal with both lasers on is seen to be on1y $26 \%$ of the undeflected ${ }^{138} 8$ a signal with both lasers off.

In order to interpret this result it is necessary to know what fraction of the atoms entering the ionizer is in the ground state and what fraction is in the $5 d^{2}$ state. Using a green probe beam in the same manner as was employed to show 6s5d metastable accumulation (Section V.G.), it was found that about $50 \%$ of ${ }^{138} \mathrm{Ba}$ the atoms in the combined deflected and undeflected beams were in the ground state after deflection. It should be noted that in this experiment the mass spectrometer detects only atoms which have made a relatively small number of absorptions and emissions and therefore have not been separated. Absorption emission cycles occur at a rate of $5 \times 10^{7} / \mathrm{sec}$ on the $5536 \AA$ transition and depopulation of the $6 \mathrm{~s} 5 \mathrm{~d}$ state occurs in approximately $10^{-7} \mathrm{sec}$. Since an atom is subject to absorption for about $5 \times 10^{-5} \mathrm{sec}$, atoms which are separ ated are 

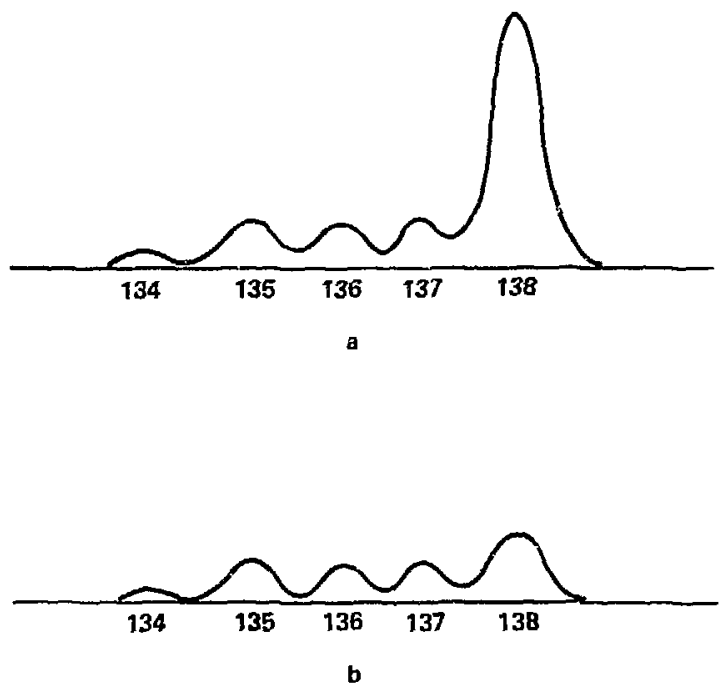

FIGURE 24: Mass analyser detecting only unseparated atoms,

a) lasers off

b) both lasers on. The $58 ? 6 \AA$ beam is telescoped to overlap $5536 \mathrm{~A}$ beam. 
likely to have made the series of transitions into the $\overline{5} \mathrm{~d}^{2}$ metastable state early in their passage through the laser beams. For this reason more than $50 \%$ of the 138 Ba atoms which are detected by the mass analyzer will be in the $5 d^{2}$ metastable level. If all of thern nter the ionizer in this state the ${ }^{138} \mathrm{Ba}$ output signal with the lasers on is more than a factor of two high due to increased ionizer efficiency which implies that less than $13 \%$ of the ${ }^{138} \mathrm{Ba}$ atoms remain unseparated. On the other hand, a 50-50 mixture of ground and metastable ${ }^{138} \mathrm{Ba}$ atoms imp $7^{\text {*es a signal which }}$ is high by at least $50 \%$ and at most $17 \%$ of the ${ }^{138} \mathrm{Ba}$ atoms remain unseparated. Thus a lower limit of $83 \%$ can be placed on the separation trificiency with metastable state depopulation compared with a lower limit of $70 \%$ without such depoputation. 


\section{EXTENSIONS OF THE PHOTODEFLECTION METHOD}

The body of the thesis has dealt with the resonant scattering photodefiection method of isotope separation. It is characterized by the fact that only the absorption process produces deflection on the average. The photodeflection process can be extended to include two processes in which both emission and absorption produce deflection. This means that the emission process is stimulated rather than spontaneous and this in turn implies that metastable accumulation can be avoided.

The first scheme is diagramatically presented in Figure 25 . In what follows, a pulsed laser instead of a $\mathrm{CW}$ laser will be discussed. Two mirrors are added to the original set up so that a pulse ca.. bounce back and forth, intersecting the atomic beam many times. After each pass, a given atom has a $50 \%$ likelihood of being in the excited state, and a $50 \%$ likelihood of being left in the ground state. If a given atom absorbs one direction and re-emits in the opposite direction a net momentum of twice $\frac{h v}{c}$ has been imparted to the atom. Furthermore, the absorbed photon has not been lost, as was the case in the resonant scattering approach, since it is re-emitted into the laser beam. In this stimulated photodeflection scheme, an atom is analogous to the drunken man in the random walk problem, staggering from the lamppost onto which he was draped. The questions in this problem is, how many steps must the drunken man take, assuming he has equal probability of surging forward or backward, in order to end up $\mathrm{N}$ sober steps away from the lamppost. The answer of course is $\mathrm{N}^{2}$ and thus for the atom, $\frac{N^{2}}{2}$ absorption-emission events are necessary to achieve an average increase in transverse momentum by $N \frac{h_{v}}{c}$. Thus, the atomic beam spreads in both directions but only the single isotope occupies the region outside the original atomic beam. Note that if the time for $\mathrm{N}^{2}$ traversals 


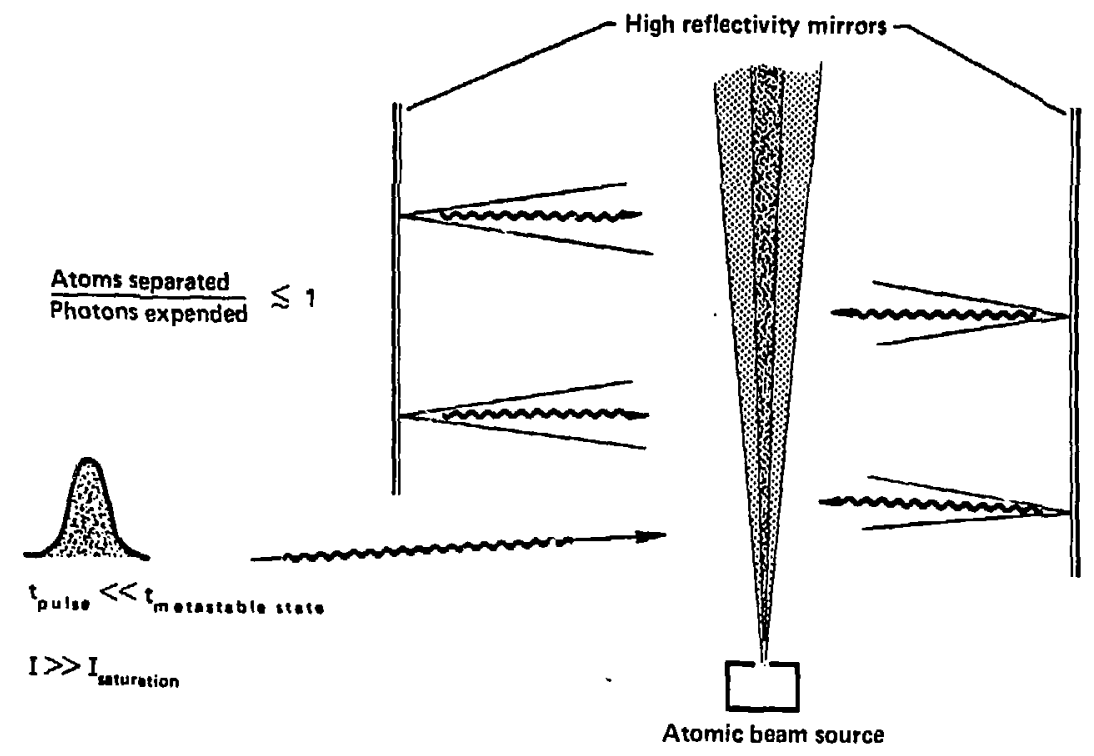

FIGURE 25: Incoherent Stimulated Photodeflection Scheme 
is much less than the time it takes for an excited atom to decay to the metastable state, this 1 imitation on $N$ goes away. It should be emphasized that only a fractional number of photons are needed per atom separated, the fraction being determined partly by the spontaneous radiation and mirror losses, but primarily by the fact that the average atom in the beam that was interacting with the laser light, carries off half of a photon of energy in residual excitation. There is of course additioral disadvantage in that not all the atoms of the isotope of interest are scavenged from the original beam, due to the probablistic nature of the spearation mechanism.

The second scheme is far more elegant and efficient than the first, but more difficult experimentally. It was very recently proposed by Szöke and Mebenzahl of Tel Aviv University ${ }^{50}$ and is illustrated in Figure $2 E$. A pulse propagates back and forth across the atomic beam as in the stimulated photodeflec'. In scheme but now, if the pulse is adiabatically frequency-modulated over the width of the atomic absorption line, not just half, but all of the atoms will be left in the excited state after the first passage of the pulse across the atomic beam. Similarly, not just half, but ail of the atoms in the beam will be returned to the ground state when the pulse traverses the beam going in the opposite direction. Arid so on. On each pass, each atom gains transverse momentum $\frac{h v}{c}$ and after an even number of traversals no atoms are left in the excited state. All of the atoms of the desired isotope are deflected and a second beam is obtained which is nearly identical to the first beam except for its isotopic composition. In order for this coherent stimulated process to work, however, the whole thing must be carried out in a time small compared to 


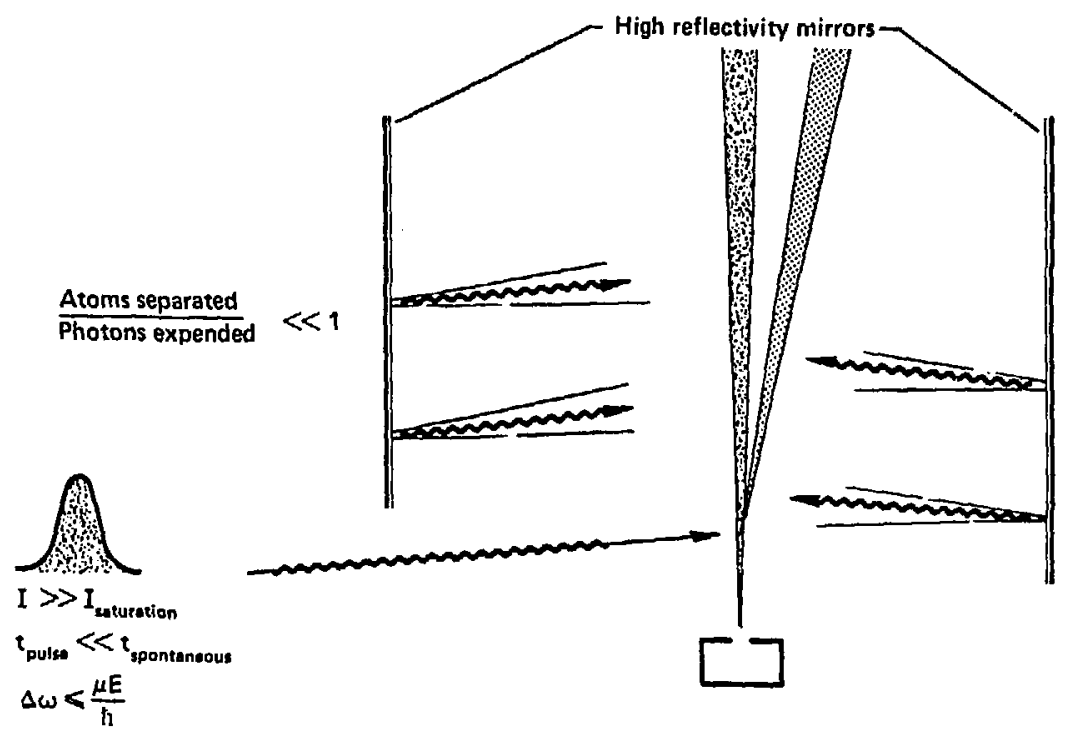

FIGURE 26: Coherent Stimulated Photodeflection Scheme 
any decay time of the atomic system. This is a somewhat more stringent condition than the time restraint $t \ll t_{\text {metastable }}$ in the stimulated photodeflection approach but then cnly $\mathbf{N}$ passes are needed instead of $N^{2}$. In this approach, the only photon costs are mirror loss and some spontaneious decay. It is emphasized that having made $N$ bounces, the laser pulse is available for recycling. 
Acknowledgments

During most of my graduate career, I have been employed at the Lawrence Livermore Laboratory of the University of California. My participation there in applications of science and technology vastly broadened the understanding of the science I acquired in the classroom. I learned numerous experimental techniques and was given use of much equipment which would have been more difficult to come by at a smaller institution.

I wish to thank all those with whom I have worked, particularly Mr. Donald E. Duerre and Mr. Joe R. Simpson who were instrumental in the successful completion of the work reported here. I would also like to thank Dr. Yu-Li Pan, with whom I worked for two years before the start of the present project and with whom I gained much of my knowledge of laser physics.

If there is one man who has been responsihie for my development as a scientist, it is Dr. Lowell L. Wood whom I have known since I was a sophomore in coilege. It was Lowell who suggested the photodeflection method of isotope separation for my dissertation experiment. I am endebted to him for his active support and collaboration throughout the present research and, indeed, throughout my undergraduate and graduate careers.

Three more acknowledgments are in order. The first is to Proressor Willard F. Libby, who has given many undergraduates including myself their first real experience in scientific research. The second is to Professor Edward Teller who, as my thesis advisor, has given his active interest and support to my research. The last is due Dr. Montgomery H. Johnson in whose classes I learned a large fraction of the physics I know. 
Finally, I wish to thank Mrs. Kathy Rieser for typing this manuscript and for helping avoid much of the chaos to which our group is prone.

During my graduate career I have received financial assistance from several sources. I would like to express my gratitude for an N.D.E.A. Title IV fellowship during my first year of graduate school and a Fannic and John Hertz Foundation Fellowship during the present year. I would also like to thank the Lawrence Livermore Laboratory and the University of California for various financial benefits, such as time off to attend class and payment of tuition, during my emplcy in the intervening vears. 
Appendix A

The separation efficiency was given on page 46 as

$$
\varepsilon=\frac{\int_{0}^{\infty} \int_{0}^{\theta_{0}} v^{3} e^{-m v^{2} / 2 k T} e^{-n(v, e) x} d \theta d v}{\theta_{0} \int_{0}^{\infty} v^{3} e^{-m v^{2} / 2 k T} d v}
$$

Evaluation of the denominator is accomplished by integration by parts:

$$
\begin{aligned}
0_{0}^{\infty} v^{3} e^{-m v^{2} / 2 k T} d v \\
=\theta_{0} \frac{2 k T}{m} \int_{0}^{\infty} v e^{-m v^{2} / 2 k T} d v \\
=\theta_{0} \frac{2 k T}{m} \int_{0}^{\infty} e^{-m v^{2} / 2 k T} d v^{2} / 2 \\
=\frac{\theta_{0}}{2}\left(\frac{2 k T}{m}\right)^{2}
\end{aligned}
$$

To evaluate the numerator, we begin with the $\theta$ integration

$$
\begin{aligned}
& \int_{0}^{\infty} \int_{0}^{\theta} v^{3} e^{-m v^{2} / 2 k T} e^{-\frac{m v c x}{h v} \theta} d \theta d v \\
&=\int_{0}^{\infty} v^{3} e^{-m v^{2} / 2 k T} \int_{0}^{\theta} e^{-\frac{m v c x}{h v} \theta} d \theta d v \\
&=\frac{h v}{m c x} \int_{0}^{\infty} v^{2} e^{-m v^{2} / 2 k T}\left(1-e^{\left.-\frac{m c x}{h v}\right)}\right) d v
\end{aligned}
$$

Then consider the first term in the above expression. Let $z=\sqrt{\frac{m}{2 k T}} v$. 
Then

$$
\begin{aligned}
\frac{h v}{m c x} \int_{0}^{\infty} v^{2} \mathrm{e}^{-m v^{2} / 2 k T} d v \\
=\frac{h v}{m c_{x}}\left(\frac{m}{2 k T}\right)^{-3 / 2} \int_{0}^{\infty} z^{2} \mathrm{e}^{-z^{2}} \mathrm{dz} \\
=\frac{h v}{n c_{x} x}\left(\frac{m}{2 k T}\right)^{-3 / 2}\left(-\left.\frac{z}{2} \mathrm{e}^{-z^{2}}\right|_{0} ^{\infty}+\frac{1}{2} \int_{0}^{\infty} \mathrm{e}^{-z^{2}} \mathrm{dz}\right) \\
=\frac{h v}{m c_{x}}\left(\frac{m}{2 k T}\right)^{-3 / 2} \frac{\sqrt{\pi}}{4}
\end{aligned}
$$

The second term in expression (1) is more difficult. We first complete the square in the exponent:

$$
\begin{aligned}
& -\frac{h_{v}}{m c_{x}} \int_{0}^{\infty} v^{2} e^{-m v^{2} / 2 k T} e^{-\frac{m \varepsilon ; \theta}{h v} v} d v
\end{aligned}
$$

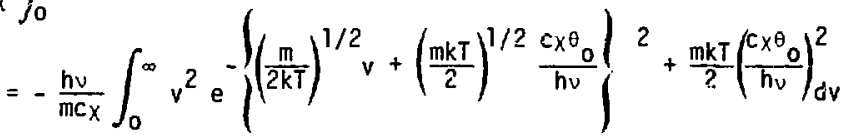

$$
\begin{aligned}
& =-\frac{h_{v}}{m c_{X}} e^{\frac{m k T}{2}\left(\frac{c_{X} \theta_{0}}{h_{v}}\right)^{2}} \int_{0}^{\infty} v^{2} e^{-y^{2}} d v
\end{aligned}
$$

We now wart to write $v^{2} d v$ in terms of the exponent $r$

$$
\gamma=\left(\frac{m}{2 k T}\right)^{1 / 2} v+\left(\frac{m k T}{2}\right)^{1 / 2} \frac{c x_{0}{ }_{0}}{h v}
$$

so that

$$
v=\left(\frac{m}{2 k T}\right)^{1 / 2} \gamma+k T \frac{c_{X} \theta_{0}}{h v}
$$


and

$$
v^{2}=\left(\frac{m}{2 k T}\right)^{-1} \gamma^{2}-\left(\frac{m}{2 k T}\right)^{-3 / 2} \frac{m c \times \theta_{0}}{h v} \gamma+\left(k T \frac{c \times 0}{h \omega}\right)^{2}
$$

Thus

$$
v^{2} d v=\left(\frac{m}{2 k T}\right)^{-3 / 2} \gamma^{2} d \gamma-\left(\frac{m}{2 k T}\right)^{-1} \frac{m c x \theta_{0}}{h \nu} \gamma d \gamma+\left(\frac{m c x \theta_{0}}{h_{\nu}} 0\right)^{2}\left(\frac{m}{2 k T}\right)^{2} d \gamma
$$

The second term in (1) now becomes

$$
\begin{aligned}
& \frac{h_{u}}{m c_{x}} e^{\frac{m k T}{2}\left(\frac{c x^{\theta} o}{h}\right)^{2}}\left(\left(\frac{m}{2 k^{T}}\right)^{-3 / 2} \int_{\alpha x}^{\infty} e^{-\gamma^{2}} \gamma^{2} d \gamma\right. \\
& -\left(\frac{m}{2 k T}\right)^{-2} \frac{m c_{x}{ }_{0}}{h \nu} \int_{\alpha x}^{\infty} e^{-\gamma^{2}} \gamma d \gamma \\
& \left.+\frac{1}{4}\left(\frac{m x_{0} 0}{h v}\right)^{2}\left(\frac{m}{2 k T}\right)^{-5 / 2} \int_{\alpha x}^{\infty} e^{-\gamma^{2}} d \gamma\right)
\end{aligned}
$$

where $\quad \alpha=\left(\frac{m}{c k T}\right)^{1 / 2} \frac{k T c \theta_{0}}{h v}=\left(\frac{m k T}{2}\right)^{1 / 2} \frac{c \theta_{0}}{h_{v}}$

Each term inside the parentines is is now evaluated in turn.

a)

$$
\begin{aligned}
\int_{\alpha}^{\infty} e^{-\gamma^{2}} \gamma^{2} d \gamma & =-\left.\frac{1}{2} e^{-\gamma^{2}}\right|_{\alpha} ^{\infty}+\frac{1}{2} \int_{x}^{\infty} e^{-\gamma^{2}} d \gamma \\
& =\frac{1}{2} \alpha x e^{-\alpha^{2} x^{2}}+\frac{1}{2} \int_{\alpha x}^{\infty} e^{-\gamma^{2}} d \gamma \\
& =\frac{1}{2} \alpha x e^{-\alpha^{2} x^{2}}+\frac{1}{2} \int_{0}^{\infty} e^{-\gamma^{2}} d \gamma-\frac{1}{2} \int_{0}^{\alpha x} e^{-\gamma^{2}} d \gamma \\
& =\frac{1}{2} \alpha x e^{-\alpha^{2} x^{2}}+\frac{\sqrt{\pi}}{4}-\frac{1}{2} \int_{0}^{\alpha x} e^{-\gamma^{2}} d \gamma
\end{aligned}
$$


b)

$$
\begin{aligned}
& \int_{\alpha x}^{\infty} e^{-\gamma^{2}} \gamma d \gamma=\frac{1}{2} \int_{\alpha x}^{\infty} e^{-\gamma^{2}} d \gamma \\
& =\frac{1}{2} \int_{\alpha}^{2} x^{2} e^{-y} d y=-\frac{1}{2} e^{-y} \int_{\alpha}^{\infty} x^{2}=+\frac{1}{2} e^{-\alpha^{2} x^{2}} \\
& \text { c) } \quad \int_{\alpha x}^{\infty} e^{-\gamma^{2}} d \gamma=\int_{0}^{\infty} e^{-\gamma^{2}} d \gamma-\int_{0}^{\alpha x} e^{-\gamma^{2}} d \gamma \\
& =\frac{\sqrt{\pi}}{2}-\int_{0}^{\alpha x} e^{-\gamma^{2}} d \gamma
\end{aligned}
$$

the entire expression now becomes

$$
\begin{aligned}
& \frac{\int_{0}^{\theta} 0 \int_{0}^{\infty} v^{3} e^{-l n v^{2} / 2 k T} e^{-n(v, \theta) x} d v d \theta}{\theta_{0} \int_{0}^{\infty} v^{3} e^{-m v^{2} / 2 k T d v}} \\
& =\frac{\sqrt{\pi}}{2 \theta_{0}}\left(\frac{m}{2 k T}\right)^{1 / 2} \frac{h v}{m c x}+\frac{1}{2}-\frac{\sqrt{\pi}}{2 \theta_{0}}\left(\frac{m}{2 k T}\right)^{1 / 2} \frac{h v}{m c x} e^{\alpha^{2} x^{2}} \\
& +\frac{e^{\alpha^{2} x^{2}}}{\theta_{0}}\left(\frac{m}{2 k T}\right)^{1 / 2} \frac{h \nu}{m c x} \int_{0}^{\alpha x} e^{-r^{2}} d \gamma-\frac{\sqrt{\pi}}{4} \frac{m c x}{h \nu} \theta_{0}\left(\frac{m}{2 k T}\right)^{-1 / 2} e^{\alpha^{2} x^{2}} \\
& +\frac{1}{2} \frac{m c x \theta}{h \nu}\left(\frac{m}{2 k T}\right)^{-1 / 2} e^{\alpha^{2} x^{2}} \int_{0}^{a x} e^{-\gamma^{2}} d \gamma
\end{aligned}
$$

let $\quad B=\left(\frac{\mathrm{m}}{2 \mathrm{kT}}\right)^{1 / 2} \frac{\mathrm{hv}}{\mathrm{mc} \theta_{0}}$

$$
\begin{aligned}
= & \frac{1}{2}+\frac{\sqrt{\pi}}{2} \frac{\beta}{x}-\frac{\pi}{2} e^{\alpha^{2} x^{2}}+\frac{\beta}{x} e^{x^{2} x^{2}} \int_{0}^{\alpha x} e^{-\gamma^{2}} d \gamma \\
& -\frac{\sqrt{\pi}}{4} \frac{x}{B} e^{\alpha^{2} x^{2}}+\frac{x}{2 B} e^{\alpha^{2} x^{2}} \int_{0}^{\alpha x} e^{-\gamma^{2}} d \gamma
\end{aligned}
$$

Substituting $\int_{0}^{\alpha \chi} e^{-\gamma^{2}} d \gamma=\frac{\sqrt{\pi}}{2}$ erf $(\alpha x)$, the above expressions becone those used in the text. 


\section{REFERENCES}

1. A. Ashkin, Phys. Rev. Lett. 25, 1321 (1970).

2. A. F. Bernhardt, D. E. Duerre, J. R. Simpson and L. L. Wood, Appi. Pnys. Lett. 25, 617 (1974).

3. J. C. Slater, "Quantum Theory of Atomic Structure. Volume I", McGraw-Hi11, 1960, pp. 159-164.

4. H. Figger and H. Walther, Z. Phys, 267, 1 (1974).

5. A. C. G. Mitchel and M. W. Zemânsky, "Resonance Radiation and Excited Atoms", Cambridge, 1971. p. 97.

6. H. Kopfermann, "Nuclear Moments", Academic Press, 1958, pp. 161-167.

7. D. J. Hughes and C. Eckart, Phys. Rev. 36, 694 (1930).

8. Reference 4, päge 167.

9. Reference 4, page 124 .

10. R. D. Ehrlich, et al, Phys. Rev. Lett. 18, 959 (1967).

11. D. A. Jackson and Duong Hong Tuan, Proc. Roy. Soc. (London), A291, 9 (1966).

12. This work

13. D. W. Steinhaus, et al, "The Emission Spectrum of Uranium between 19,080 and $30,261 \mathrm{~cm}^{-1 "}$, Los Alamos Scientific Laboratory, Report LA-4944 (1972).

14. N. Ramsey, "Molecular Beams", Oxford, 1956, page 21.

15. J. D. Jackson, "Classical Electrodynamics", John Wiley \& Sons, 1962 , p. 605.

16. G. Herzberg, "Atomic Spectra and Atomic Structure", Dover, 1944, pp. 154-159.

17. L. D. Landau and E. M. Lifschitz, "Mechanics", Perganon Press 1960, Chapt. IV.

18. For a general treatise on Vander Waal interactions, see H. Margenau and N. R. Kestner, "Theory of Intermolecular Forces", Perganon Press 1969.

19. F. London, Z. Phys. 63,245 (1930).

20. R. V. Ambartzumian and V. S. Letokhov, Appl. Opt. 11, 354 (1972). 
21. U. Brinkman, W. Hartig, H. Telle and H. Walther, Appl. Phys. $\underline{5}$, 109 (1974).

22. S. A. Tuccio, J. W. Dubrin, 0. B. Peterson and B. B. Snavely, "Two Step Selective Photoionization of $235 \mathrm{U}$ in Uranium Vapor", paper Q-14, VIII International Quantum Electronics Conference, San Francisco, June 1974.

23. L. O. Dickie and F. M. Kelly, Can. J. Phys, 48, $879(1970)$.

24. M. W. Swagel and A. Lurio, Phys. Rev. 169, 114 (1968).

25. Reference 13, Chapter 9.

26. P. McCavert and E. Trefftz, J. Phys. B7, 1270 (1974).

27. B. M. Miles and W. L. Hiese, U. S. Natl. Bur. Stand. Tech. Note 474 .

28. L. O. Dickie and F. M. Kelly, Can. J. Phys. 49, 1098 (19?1).

29. L. O. Dickie and F. M. Kelly, Can. J. Phys. 49, 2630 (1971).

30. C. E. Moore, "Atomic Energy Levels, Volume III", NSRDS-NBS 35 December 1971.

31. R. L. Barger, M. S. Sorem and J. L. Hall, Appl. Phys. Lett. 22, $573(1973)$.

32. F, Y. Wu, R. E. Grove andS. Ezekie?, Appl. Phys. Lett. 25, 73 (1974).

33. B. B. Snavely, Proc. IEEE, 57, 1374 (1969).

34. B. B. Snavely and 0. G. Peterson, IEEE J. Quant. Elec. QE-4, $540(1963)$.

35. For a more complete description of the laser design see S. A. Tuccio and F. C. Strome, Jr., App 7. Optc. 11, 6f (1972).

36. H. W. Kogelnik and T. Li, Appl. Opt. 5 , 1550 (1966).

37. See, for example, M. Born and E. Wolf, "Principles of Optics", Third Edition, Perganon Press Section 4.7.2.

38. S. P. Davis, Appl. Opt. 2, 727 (1963).

39. W. G. Wagner and G. Birnbaum, J. Appl. Phys. 32, 1185 (1961).

40. C. M. Harris and C. E. Crede, "Shock and Vibration Handbook, Volume 2", McGraw-Hill, 1961.

41. R. Wallenstein and T. H. Hansch, Appl. Opt. 13, 1625 (1974). 
42. For a more complete description of the laser's design and performance, see H. W. Kogelnik, E. P. Ippen, A. Dienes, and C. W. Shank, IEEE J. Quant. Eler. QE-8, 373 (1972) and J. M. Yarborough, App1. Phys. Lett. 24, $629(1974)$.

43. A. Bloom, J. Opt. Soc. Am. 64, 447 (1974).

44. In a paper published since completion of the present work W. Rasmussen, R. Schieder and H. Wal ther (0pt. Comm. 12,315 (1974)) have determined the positions of the odd isotope transitiuns of barium. They find that the $\mathrm{F}=1 / 2$ peaks of $135 \mathrm{Ba}$ and $137_{\mathrm{Ba}}$ are located at about 525 and 535 MHz respectiveiy. Their results for the other odd isotope peaks are in excellent agreement with those reported here.

45. M. Abramowitz and 1. A. Stegun, "Handbook of Mathematical Functions", U. S. Government Printing Office, June 1964, page 298.

46. A. C. Gallagher and G. York, Rev. Sci. Instrum. 45, 662 (1974).

47. The values for the oscillator strengths vary from author to author (see references 23,25 , and 41 ) but the ratio of the two is about the same.

48. N. P. Penkin, J. Quant. Spectr. 4, 41 (1964).

49. A. Szoke and 1. Nebenzahl, Appl. Phys. Lett. 25, 327 (1974). 